\title{
Speckle interferometry at SOAR in 2012 and $2013^{\dagger}$
}

\author{
Andrei Tokovinin \\ Cerro Tololo Inter-American Observatory, Casilla 603, La Serena, Chile \\ atokovinin@ctio.noao.edu \\ Brian D. Mason \& William I. Hartkopf \\ U.S. Naval Observatory, 3450 Massachusetts Ave., Washington, DC, USA \\ bdm@usno.navy.mil, wih@usno.navy.mil
}

\begin{abstract}
We report the results of speckle runs at the 4.1-m Southern Astronomical Research (SOAR) telescope in 2012 and 2013. A total of 586 objects were observed. We give 699 measurements of 487 resolved binaries and upper detection limits for 112 unresolved stars. Eleven pairs (including one triple) were resolved for the first time. Orbital elements have been determined for the first time for 13 pairs; orbits of another 45 binaries are revised or updated.
\end{abstract}

Subject headings: stars: binaries

\section{Introduction}

Knowledge of binary-star orbits is of fundamental value to many areas of astronomy. They provide direct measurements of stellar masses and distances, inform us on the processes of star formation through statistics of orbital elements, and allow dynamical studies of multiple stellar systems, circumstellar matter, and planets. A large fraction of visual binaries are late-type stars within $100 \mathrm{pc}$, amenable to searches for exo-planets. However, the current orbit catalog contains some poor or wrong orbital solutions based on insufficient data. To improve the situation, we provide here new observations, revise some orbits, and compute new ones.

This paper continues the series of speckle interferometry observations published by Tokovinin (2010, hereafter TMH10), Tokovinin et al. (2010),

\footnotetext{
${ }^{\dagger}$ Based on observations obtained at the Southern Astrophysical Research (SOAR) telescope, which is a joint project of the Ministério da Ciência, Tecnologia, e Inovação (MCTI) da República Federativa do Brasil, the U.S. National Optical Astronomy Observatory (NOAO), the University of North Carolina at Chapel Hill (UNC), and Michigan State University (MSU).
}

Hartkopf et al. (2012), and Tokovinin (2012). We used the same equipment and data reduction methods. All observations were obtained with the 4.1-m SOAR telescope located at Cerro Pachón in Chile. Our program is focused on close binaries with fast orbital motion, where the frequency of measurements (rather than the time span) is critical for orbit determination. Some of those binaries were discovered by visual observers, but most are recent discoveries made by the Hipparcos mission and by speckle interferometry, including our work at SOAR. Spectroscopic orbits are available for several fast nearby binaries resolved here. In addition, we measured close binaries with known orbits to verify and improve them when necessary, and wider pairs for calibration and quality control.

Data on binary-star measures and orbits are \&idartakp the Washington Double Star Catalog, WDS (Mason et al. 2001)1 1 and associated archives such as the $4^{\text {th }}$ Catalog of Interferometric Measurements of Binary Stars, INT4 (Hartkopf. McAlister \& Mason 2001) and the

\footnotetext{
${ }^{1}$ See current version at http://ad.usno.navy.mil/wds/
} ${ }^{2}$ http://ad.usno.navy.mil/wds/int4.html 
$6^{\text {th }}$ Orbit Catalog of Orbits of Visual Binary Stars, VB6 (Hartkopf. Mason \& Worlev 2001) 3 These resources are extensively used here.

Section 2 recalls the observing technique and presents new measures, discoveries, and nonresolutions. New and updated orbits of 13 and 45 systems are given in Section 3 and Section 4 respectively.

\section{New speckle measures}

\subsection{Instrument and observing method}

The observations reported here were obtained with the high-resolution camera (HRCam) - a fast imager designed to work at the 4.1-m SOAR telescope (Tokovinin \& Cantarutti 2008). For practical reasons, the camera was mounted on the SOAR Adaptive Module (SAM, Tokovinin et al. 2008). However, the laser guide star of SAM was not used; the deformable mirror of SAM was passively flattened and the images are seeing-limited. The SAM module corrects for the atmospheric dispersion and helps to calibrate the pixel scale and orientation of HRCam, as explained below. The transmission curves of HRCam filters are given in the instrument manual W. We used mostly the Strömgren $y$ filter $(543 / 22 \mathrm{~nm})$ and the nearinfrared $I(774 \mathrm{~nm})$ filter.

Observation of an object consists of accumulation of 400 frames of 200x200 pixels each with exposure time of $20 \mathrm{~ms}$ or shorter. Frames of 400x400 pixels were recorded for pairs with separation larger than 1 .'5. Each object was normally recorded twice and these two image cubes were processed independently. Parameters of resolved binary and triple systems are determined by fitting a model to the power spectrum, as explained in TMH10.

\subsection{Calibration of scale and orientation}

The star light reaches HRCam after reflections from two non-rigid mirrors - the thin active primary mirror of SOAR and the deformable mirror of SAM. Both can affect the plate scale. We calibrated the transfer optics of the SAM instrument

\footnotetext{
${ }^{3}$ http://ad.usno.navy.mil/wds/orb6.html

${ }^{4}$ http://www.ctio.noao.edu/new/Telescopes/SOAR/Instruments/SAM/archive/hrcaminst.pdf
}

by imaging a single-mode fiber located at the telescope focus (mounted on the SAM guide probe). The fiber was translated by a micrometer stage, allowing us to accurately determine the detector orientation relative to the instrument frame and the pixel size in microns projected from the detector to the SOAR focal plane. The pixel size at focus is $5.01 \mu \mathrm{m}$, stable throughout the runs to better than $0.5 \%$. Considering this stability, we adopted a fixed pixel scale of $15.23 \mathrm{mas} /$ pixel as in the previous papers of this series.

The same internal calibration was applied to the CCD imager, SAMI, which is part of the SAM Instrument. It has $4096 \times 4112$ pixels and covers a $3^{\prime}$ square field. Thus, both HRCam and SAMI are inter-calibrated. Selected sky images taken with SAMI during the same runs were corrected for the optical distortion in SAM and calibrated against known positions of stars using the autoastrometry too 5 and the 2MASS catalog. The pixel scale of SAMI and its orientation were thus determined and then translated to the orientation of HRCam. We noticed that the orientation of SAM was stable during each run to 0.1 , but changed between the runs, although the instrument was not dismounted from the SOAR Nasmyth rotator. Therefore, the angle of the SOAR rotator was not very stable. Moreover, it should depend on the telescope orientation if the telescope pointing model is not perfect. With this caveat in mind, we estimate the accuracy of the angle calibration to be about 0.5 .

Table 1 lists the observing runs, the angular offsets $\theta_{0}$ determined for each run, and the number of objects covered. In February and September 2013, we used the telescope time allocated for this program. The time allocation in June 2013 was lost to clouds almost entirely. The remaining data were collected during commissioning runs of the SAM instrument, as a backup program. On three of those occasions, we used only few available hours, but a whole night was spent in December 2012.

\subsection{Impact of telescope vibrations}

Previous work with HRCam, SAM, and other SOAR instruments established that the telescope vibrates with the power-line frequency of $50 \mathrm{~Hz}$ and the rms amplitude reaching 40 mas in the worst cases. The optical axis moves on an elliptical

${ }^{5}$ See http://www. astro.caltech.edu/ \{\} dperley/programs/auto\discretionary \{ 
TABLE 1

OBSERVING RUNS

\begin{tabular}{lrr}
\hline \hline \multicolumn{1}{c}{ Dates } & \multicolumn{1}{c}{$\theta_{0}$} & $N_{\text {obj }}$ \\
\hline Oct 29-30, 2012 & 2.10 & 19 \\
Dec 1-2, 2012 & 0.08 & 144 \\
Jan 28-29, 2013 & 1.50 & 11 \\
Feb 15-16, 2013 & -1.00 & 268 \\
Mar 27, 2013 & -0.21 & 41 \\
Jun 22, 2013 & -0.21 & 38 \\
Sep 25-27, 2013 & -0.66 & 174 \\
\hline
\end{tabular}
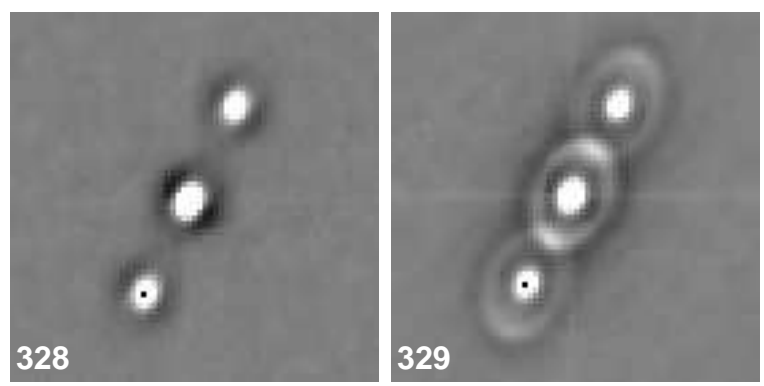

Fig. 1.- Auto-correlation functions of the binary star HU 304 with separation of 0 "'288, computed from image cubes \#328 (exposure $5 \mathrm{~ms}$, left) and \#329 (exposure $20 \mathrm{~ms}$, right). The black dot marks the companion.

path with variable elongation and amplitude; the amplitude increases with the zenith distance. The vibrations are not stationary. Attempts to locate their source (e.g. compressors of the closed-cycle coolers) have, so far, not produced definitive results.

During the standard exposure time of $20 \mathrm{~ms}$, one full vibration period is sampled, the speckles are blurred, and the resolution and sensitivity are seriously degraded. Using a shorter exposure time of $5 \mathrm{~ms}$ helps to recover the signal, at the cost of reduced flux. Figure1 1llustrates the effect of shortening the exposure time by two observations of the same binary star obtained sequentially under the same conditions. The elliptical motion of the SOAR's optical axis produces characteristic "disks" which sometimes blur the speckle completely.

We used the exposure time of $5 \mathrm{~ms}$ and went as short as $2 \mathrm{~ms}$ when possible. Fainter stars were observed with the longer exposure of $20 \mathrm{~ms}$, and in this case the success depended on the presence and amplitude of the vibrations. The February 2013 run was particularly affected, while in September 2013 the vibrations were less intense.

The faintest resolved binary is HIP $48273 \mathrm{~B}=$ RAO 90 (WDS J09505+0421) at $V=12.1$. It was observed in the $I$ filter with 20-ms exposure time; the signal is affected by vibrations. The next faintest pair is KUI 41 (WDS J09310-1329) at $V=10.7$, observed again in $I$, but with a 5 -ms exposure. With a normal 20-ms exposure, stars $1.5^{m}$ fainter could be recorded. The $2 \times 2$ binning of the detector helps to increase the sensitivity for faint stars while under-sampling the speckle, but it was not used here.

\subsection{Data tables}

Table 2 lists 699 measures of 487 resolved binary stars and sub-systems, including newly resolved pairs. The format did not change from the previous papers. The columns of Table 2 contain (1) the WDS designation, (2) the "discoverer designation" as adopted in the WDS, (3) an alternative name, mostly from the Hipparcos catalog, (4) Besselian epoch of observation, (5) filter, (6) number of individual data cubes, $(7,8)$ position angle $\theta$ in degrees and internal measurement error in tangential direction $\rho \sigma_{\theta}$ in mas, $(9,10)$ separation $\rho$ in arcseconds and its internal error $\sigma_{\rho}$ in mas, and (11) magnitude difference $\Delta m$. An asterisk follows if $\Delta m$ and the true quadrant are determined from the resolved long-exposure image; a colon indicates that the data are noisy and $\Delta m$ is likely over-estimated (see TMH10 for details). Note that in the cases of multiple stars, the positions and photometry refer to the pairings between individual stars, not with photo-centers of sub-systems.

For stars with known orbital elements, columns (12-14) of Table 2 list the residuals to the 
ephemeris position and the references to the orbits.

We did not use image reconstruction and measure the position angles modulo $180^{\circ}$. Plausible quadrants are assigned on the basis of orbits or prior observations, but they can be changed if required by orbit calculation. For triple stars, however, both quadrants of inner and outer binaries have to be changed simultaneously; usually the slowly-moving outer pair defines the quandant of the inner sub-system without ambiguity.

Our software does not have capability of fitting models of power spectra with more than three components. Sub-systems in the resolved quadruple stars RST 244Ba,Bb (WDS J07185-5721) and MLO 3Ba,Bb (WDS J13147-6335) were measured crudely using the peaks in the autocorrelation functions.

Table 3 contains the data on 112 unresolved stars, some of which are listed as binaries in the WDS or resolved here in other filters. Columns (1) through (6) are the same as in Table 2, although Column (2) also includes other names for objects without discoverer designations. Columns $(7,8)$ give the $5 \sigma$ detection limits $\Delta m_{5}$ at 0.15 and $1^{\prime \prime}$ separations determined by the procedure described in TMH10. When two or more data cubes are processed, the best detection limits are listed.

\subsection{New pairs}

For the reader's convenience, we extracted data on the binaries resolved here for the first time into Table 4. There are 11 objects, some of which are newly resolved visual triples. As in the previous runs, we tried to observe nearby solar-type dwarfs that are known to be binary by their variable radial velocity $(\mathrm{RV})$, mostly from the work by Nordström et al. (2004), or by astrometric acceleration (Makarov \& Kaplan 2005). This continues the work on resolving astrometric binaries that was done at SOAR and at Gemini (Tokovinin et al. 2012, 2013). Comments on individual stars follow.

02321-1515 = HIP 11783 is an F5V dwarf at $27 \mathrm{pc}$. It has variable RV and astrometric acceleration. The companion was resolved only in the $I$ band. The star is bright, $V=4.74^{m}$; it was observed through clouds when no other program objects could be accessed. There is also a common proper motion companion B = HIP 11579 of spectral type $\mathrm{K} 2.5 \mathrm{~V}$ located at $345^{\prime \prime}$ and 252.5 from A (Tokovinin \& Lépine 2012), making the whole system triple. The proper motions of A and B differ slightly, possibly because of the inner system $\mathrm{Aa}, \mathrm{Ab}$ with an estimated period of $\sim 10 \mathrm{yr}$.

$\mathbf{0 4 0 0 7}+\mathbf{2 0 2 3}=$ HIP 18719 is a spectroscopic binary in the Hyades with period $16.7 \mathrm{yr}$, estimated semi-major axis of 0 .'12 (Griffin 2012), and astrometric acceleration. The secondary lines were detected by Bender \& Simon (2008), who evaluated the mass of the secondary component at $0.4 \mathcal{M}_{\odot}$. The object was tentatively resolved with Robo-AO (Riddle et al. 2014) in 2012.757 at $342^{\circ}, 0^{\prime \prime} .142$ and $\Delta i=2.8$. The resolution here is secure, at smaller separation and a very different position angle of $96^{\circ}$ (or $276^{\circ}$ ) with $\Delta I=3.6$.

07435+0329 = HIP $\mathbf{3 7 6 4 5}$ has a variable RV. According to D. Latham (private communication), it is a single-lined binary with a period on the order of $35 \mathrm{yr}$. Such a period corresponds to a semi-major axis of $0 . \prime 27$, of the same order as the measured separation. This is a triple system with physical companion B at 9.'6 (AB is STF 1134, discovered in 1832). The more distant companion $\mathrm{C}$ listed in the WDS is optical, as evidenced by the motion of AC since 1906. Although the system AC is designated as STF 1134AC, it was actually first measured in 1906 by Burnham (1913).

08391-5557 = HIP 42424 is the known binary HU 1443, measured here at 0". 917 separation. Its secondary component turns out to be a new pair $\mathrm{Ba}, \mathrm{Bb}$ with 0.'07 separation. This example shows the power of speckle interferometry at a 4$\mathrm{m}$ telescope. The outer pair AB was observed with speckle interferomety by E. Horch et al. (2006) at smaller telescopes that are sufficient for its resolution, but the close sub-system escaped detection until now.

09086-2550 = HIP 44874 is similar to the previous object: the known pair RST 2610 at 1".78 hosts the new close 0 .' 12 binary Ba,Bb. The A component is itself a close pair with variable RV and astrometric acceleration. It was already observed at SOAR and found unresolved (also unresolved here). The orbital period and mass ratio of the sub-system $\mathrm{Aa}, \mathrm{Ab}$ thus remain indeterminate so far. In contrast, the new sub-system Ba,Bb has an estimated period of $\sim 15 \mathrm{yr}$ and component masses of 0.6 and $0.5 \mathcal{M}_{\odot}$ estimated from their 
luminosity. The whole system is therefore a $2+2$ quadruple.

$09383+0150=$ TOK 358 was also resolved with Robo-AO (Riddle et al. 2014) in 2013.0526 at $345^{\circ} .1,0^{\prime \prime} 447$ and $\Delta i=2.34$.

$11192-1950=$ RV Crt $=$ HD 98412 is an eclipsing binary of $\beta$ Lyr type, spectral type F8. The existence of the tertiary companion was suggested from the eclipse timing by T. Armond (private communication). The object was observed on her request and resolved into a $00^{\prime \prime} 05$ pair of nearly equal stars.

11420-1701 = HIP 57078 unexpectedly turned out to be a triple star. This is an F5V dwarf with astrometric acceleration (Makarov \& Kaplan 2005), presumably produced by the newly resolved 0'14 pair $\mathrm{Aa}, \mathrm{Ab}$ with an estimated period of $\sim 20$ yr. In addition, we see a distant companion $\mathrm{B}$ at 1 '! 11 . The physical nature of B is yet to be confirmed by repeated measurement within a year, but is likely, considering the low density of background stars around this target.

$\mathbf{1 2 5 0 9}-\mathbf{5 7 4 3}=$ HIP 62699 is another nearby dwarf with astrometric acceleration and variable RV resolved here at $0 . \prime 19$. The estimated period is $\sim 25 \mathrm{yr}$.
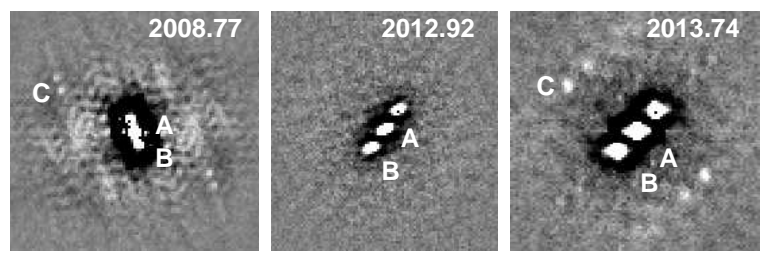

Fig. 2.- Auto-correlation functions of the resolved triple system VOU 35 observed on three epochs at SOAR. North is up, East to the left, the scale is approximate.

$21368-3043=$ VOU 35 AB $=$ HIP 106701 is a G5V dwarf with Hipparcos parallax of 16.1 mas. The projected separation of $\mathrm{AB}$ indicates a probable orbital period of about $20 \mathrm{yr}$ (see the orbit with $P=19.8 \mathrm{yr}$ in Table 6, Figure 14). We detect another faint component $\mathrm{C}$ at $0^{\prime \prime} 45$ and 53.5 from A. Re-examination of archival speckle data show that the component $\mathrm{C}$ was not seen on 2008.54 (it was fainter than the detection limit), but was present on 2008.7669 at approximately 62.9 and $0^{\prime \prime} 475$ (in the $y$ filter). However, the next observation on 2012.923 with good signal-tonoise ratio shows no trace of the $\mathrm{C}$ component, while it is securely detected now (Figure 2).

The multiple system HIP 106701 has been detected in X-rays (see also Torres et al. 2006). The disappearance of $\mathrm{C}$ in 2012.92 could be caused by its variability (e.g. eclipses). The projected separation of $\mathrm{AC}$ is $\sim 30 \mathrm{AU}$ and implies a period on the order of $100 \mathrm{yr}$. The observed motion of AC $\left(10^{\circ}\right.$ in $\left.5 \mathrm{yr}\right)$ does not contradict this estimate. If $\mathrm{C}$ were some unrelated background star, the proper motion of A ( 0 '. 11 per yr) would have drastically changed the configuration of $\mathrm{AC}$ in 5 yrs.

Interestingly, there is another star in the 2MASS catalog at 234.0, 11."2 from A. Its infrared magnitudes match a dwarf of $\sim 0.65 \mathcal{M}_{\odot}$ at the same distance. This additional component D is visible at similar relative position in the saturated DSS image. Considering also low crowding, we tentatively conclude that the pair $\mathrm{AD}$ is physical and that the whole system is at least quadruple. For completeness, we list the AD pair in Table 4 .

\subsection{Comments on individual objects of in- terest}

Potentially spurious binaries. A binary system can become too close and unresolvable when it goes through periastron. It is expected to re-appear after a few years if the estimated orbital period is short. Repeated observations of several promising candidates at SOAR failed to resolve them, however, despite much improved resolution and dynamic range of speckle interferometry in comparison with the visual observations used to discover these binaries. Table 5 lists several such cases, adding to the list of mysterious "ghosts" in Tokovinin (2012). It gives the year of last measurement according to the WDS, the period of speckle non-resolution, and the number $N_{\mathrm{UR}}$ of negative speckle observations. The orbit with 21-yr period computed for B 594 by Norro (1983) predicts its separation between $00^{\prime \prime} 07$ and 0 ". 12 during the period of non-resolutions at SOAR.

Reversed quadrant of the orbit is evidenced in some resolved triple systems where the orientation of the inner (fast) pair is determined by the outer (slow) binary. In these cases, reversal of the observed quadrant (allowed in classical speckle) is not possible; instead, the orbital 
node must be changed by $180^{\circ}$. Such triples here are FIN 337BC (WDS J01198-0030), FIN 308AB (WDS J10282-2548), and RBT 1Aa,Ab (WDS J14038-6022).

02426-7947 = HIP 12654 is the acceleration binary TOK 362 resolved with NICI (Tokovinin et al. 2013). The new measure confirms the hypothesis that the pair opens up. Note that the secondary component is red: $\Delta V=4.2$, $\Delta I=2.6$, while $\Delta K=0.61$. The secondary could itself be a close pair of M-dwarfs. The large $\Delta V$ and presumably close separation in 1991 help to understand why this pair was not resolved by Hipparcos.

04311-4522 = HIP 21079 is the acceleration binary TOK 208 resolved in Tokovinin et al. (2012). The wide separation implies a long estimated orbital period of $\sim 600 \mathrm{yr}$, making it difficult to explain the acceleration, unless the system is triple.

06454-3148 = HIP 32366. We discovered independently the close pair $\mathrm{Ba}, \mathrm{Bb}$, converting this nearby solar-type dwarf into a triple system. In fact, this system was resolved earlier by Ehrenreich et al. (2010). They even suggested a preliminary circular orbit of $\mathrm{Ba}, \mathrm{Bb}$ with a period of $3.5 \mathrm{yr}$, based on several measurements. The small astrometric acceleration of A could be caused by its attraction to component B.

06573-4929 = RST 5253 AC. The wide companion $\mathrm{C}$ was discovered at SOAR on 2010.97 at $1^{\prime \prime} .05,236$.9; the quadrant published in Hartkopf et al. (2012) was chosen wrongly. It is measured here at $1^{\prime \prime}$.06 and 238.6. The quadrant is now confirmed from the direct image and matches the orbit of the inner pair AB.

09383+0150 = HIP 47292 is an acceleration binary, also resolved in 2013.05 with RoboAO (Riddle et al. 2014).

14038-6022 = VOU 31 and RBT 1 $\mathbf{A a}, \mathbf{A b}$ is the spectacular triple system $\beta$ Cep.

\section{First orbits}

In this Section, we derive first orbits for some binaries or sub-systems observed here. This continues the work of Hartkopf et al. (2012) on cleaning and improving the VB6 catalog. We refer to that paper where the method of orbit calculation is described. The orbital elements of
13 pairs are listed in Table 6 . As a consistency check, the mass sum resulting from the new orbits and the Hipparcos parallax is given. We discuss briefly some of these objects in the remainder of this Section. Figures 3-15 show the new orbits. In each of those figures, speckle and other highresolution measures are plotted as filled circles, the measures from this work as filled stars, micrometric observations as plus signs, and the Hipparcos observations as filled diamonds. A line connects each measure to its predicted position on the orbital ellipse. The dot-dashed line passing through the primary indicates orbital nodes, the grey circle shows diffraction limit of the 4.1-m telescope, with the scale in arcseconds shown on both axes. The orientation and the direction of motion are indicated in the lower right corner of each figure.

02022-2402 = TOK $41 \mathrm{Ba}, \mathrm{Bb}$ is the secondary sub-system with nearly equal components in the triple star HIP 9497. The 138-yr orbit of the outer pair $\mathrm{AB}=\mathrm{HDS} 272$ (Hartkopf \& Mason 2011) is still preliminary and corresponds to a mass sum of $11.4 \mathcal{M}_{\odot}$. The 14 -yr orbit of $\mathrm{Ba}, \mathrm{Bb}$ is not yet fully covered and also preliminary. Further speckle monitoring will lead to the reliable orbits that will allow dynamical analysis of this interesting triple system with weak hierarchy, i.e. a small ratio of outer and inner periods.

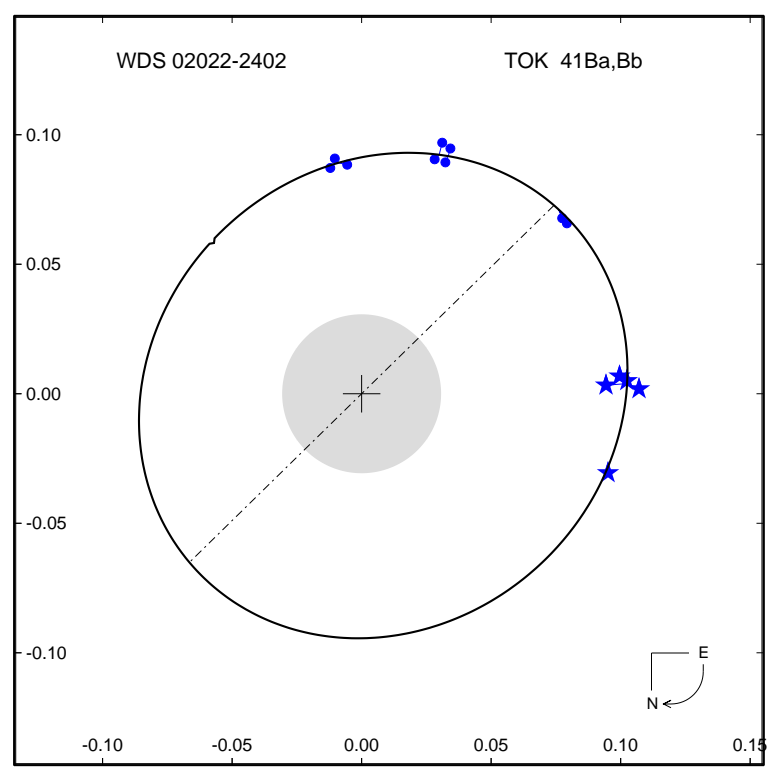

Fig. 3.- Orbit of TOK $41 \mathrm{Ba}, \mathrm{Bb}$. 
02572-2458 = BEU $4 \mathrm{Ca}, \mathrm{Cb}$ is a spectroscopic pair belonging to the quadruple system ADS 2442, known also as GJ 120.1. The components $\mathrm{AB}$ are HD $18455=\mathrm{HIP} 13772=$ BU 741 (also measured here), the component $\mathrm{C}$ is HD $18445=$ HIP 13769, K2V. It was announced as a spectroscopic binary by Duquennoy \& Mayor in 1991. Some elements of its spectroscopic orbit were published by Halbwachs et al. (2000); they mention orbit publication in a "forthcoming paper" which has not yet appeared. The low velocity amplitude hinted at a secondary component of planetary or brown-dwarf mass. However, the system was resolved by Beuzit et al. (2004) in 2000.6. The large mass of $\mathrm{Cb}$ was also established by Reffert \& Quirrenbach (2011) from the Hipparcos astrometry. We see now that the orbit is oriented almost face-on, explaining the low RV amplitude (which is further reduced by line blending). In orbit fitting, we fixed some elements known from the spectroscopy and the poorly-constrained inclination.

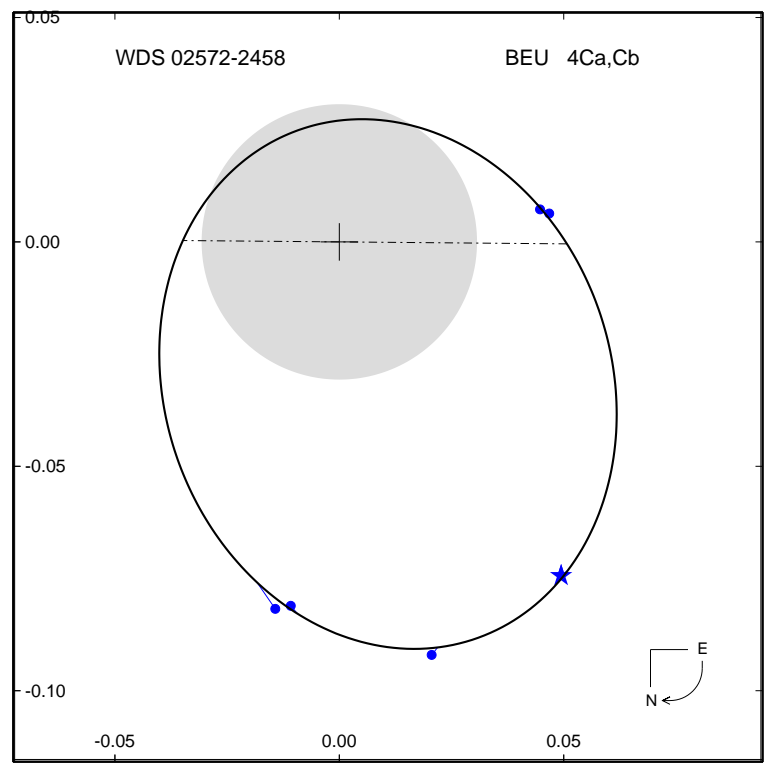

Fig. 4.- Orbit of BEU $4 \mathrm{Ca}, \mathrm{Cb}$.

05072-1924 = FIN $376=$ HIP $23818=$ HD 33095 is a double-lined spectroscopic binary with a period of $3.9 \mathrm{yr}$ according to D. Latham (private communication). The short period explains why no visual orbit has been computed so far, as the orbital coverage was insufficient. The orbit in Table 6 is derived by combining radial ve-

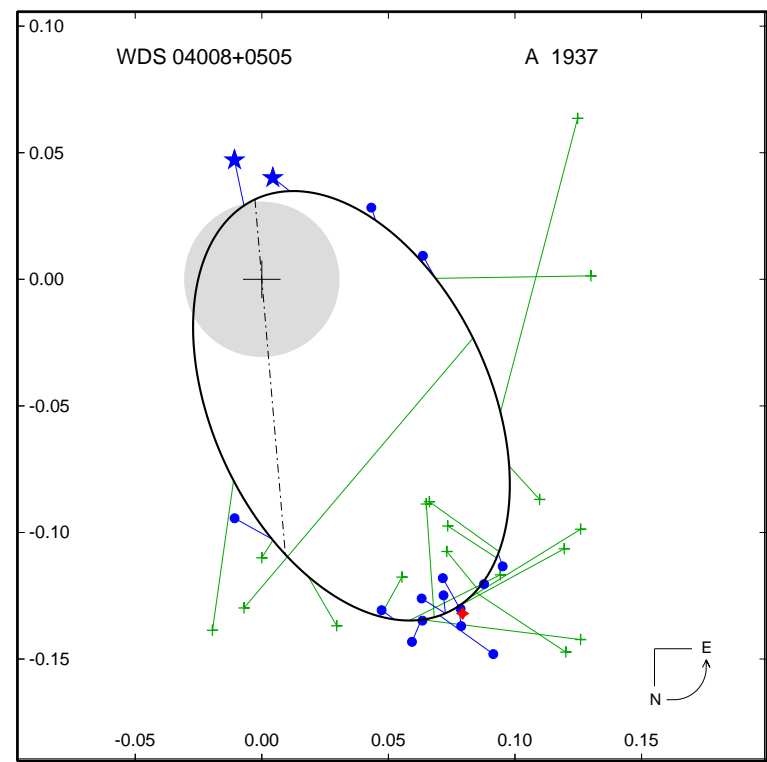

Fig. 5.- Orbit of A 1937

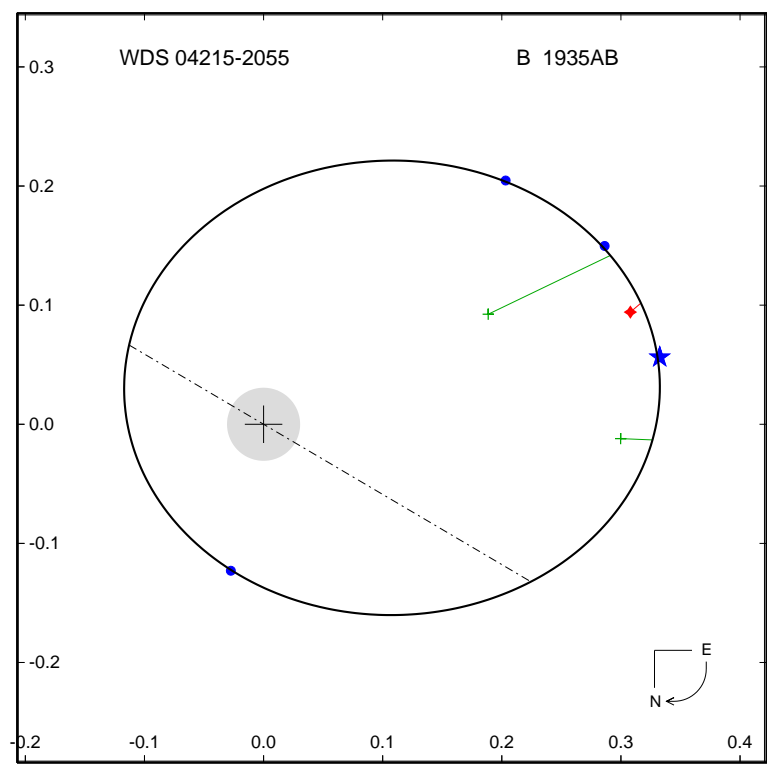

Fig. 6.- Orbit of B 1935

locities of the two components kindly made available to us by D. Latham with the speckle and visual measurements, which explains the small errors. We do not publish here the spectroscopic elements and the resulting masses, deferring this analysis to a future paper.

07478-0332 = RST $4375=$ HIP 38039. The speckle measures from the 1990s appear to be 


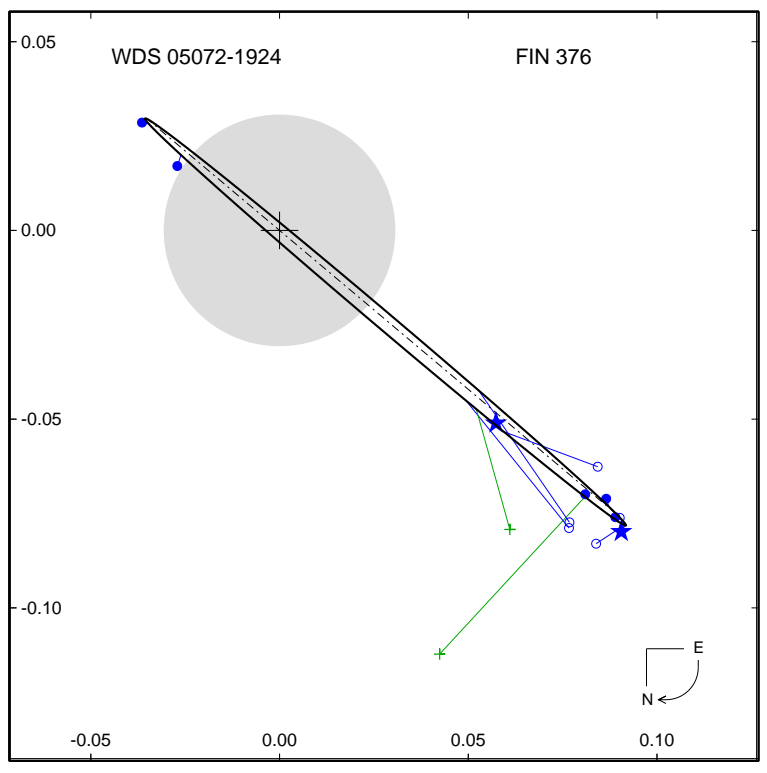

Fig. 7.- Orbit of FIN 376.

located on a straight line, rather than on an ellipse (Figure 8). The orbit is still very preliminary. The mass sum of $6.5 \mathcal{M}_{\odot}$ seems a bit too large for the spectral type A0. Considering the large deviation of the Hipparcos measurement from the speckle data, we might suspect inaccurcy of its parallax.

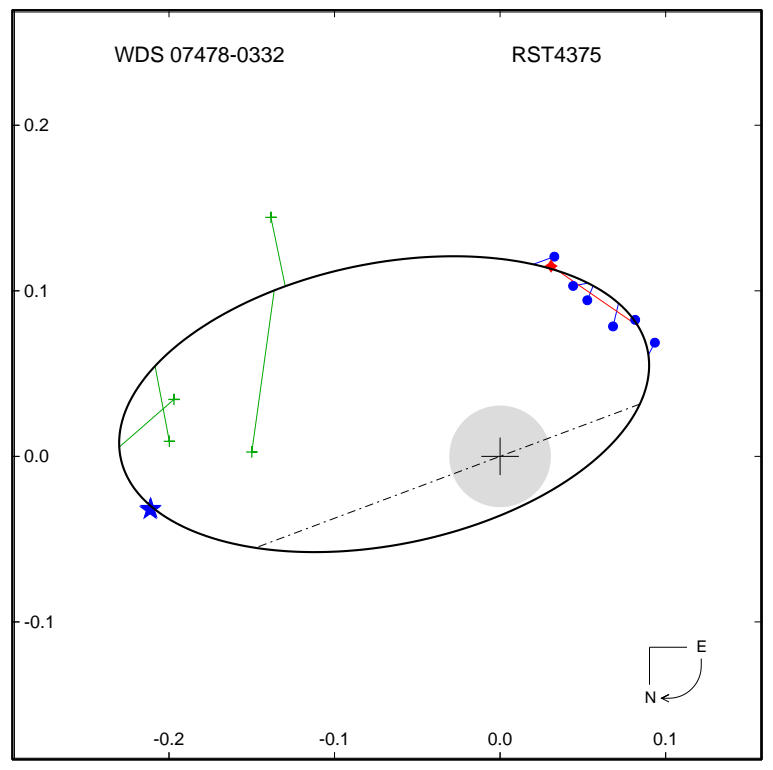

Fig. 8.- Orbit of RST 4375
09191-4128 = CHR $239=$ HIP 45705. This first orbit appears quite reliable already and gives a reasonable mass sum (spectral type G2V). This is also an acceleration binary in Hipparcos.

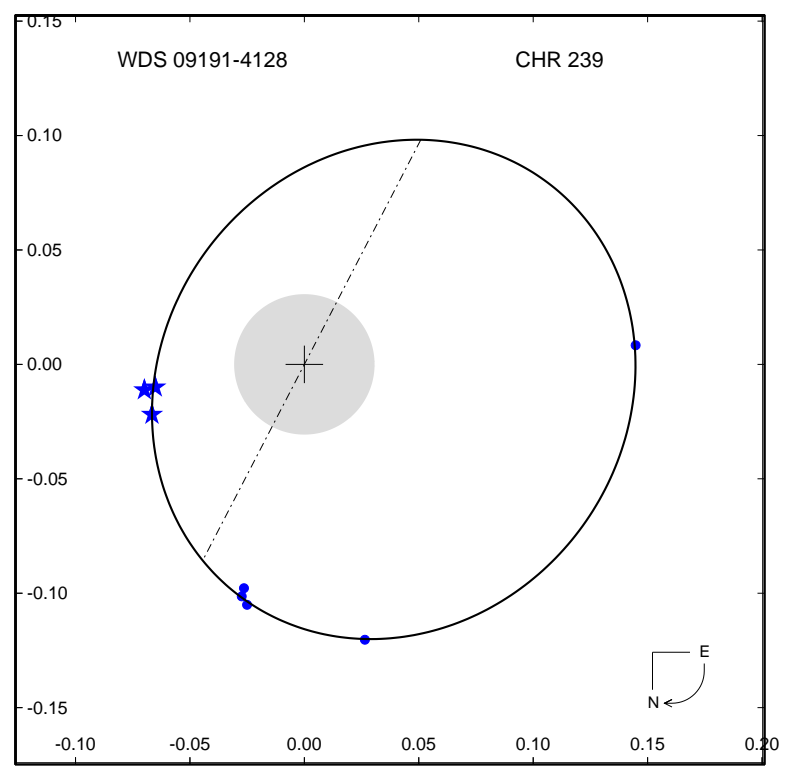

Fig. 9.- Orbit of CHR 239

$11514+1148=$ HDS $1672=$ HIP 57821. The 54-yr orbit is not yet fully covered and remains preliminary. The mass sum matches spectral type F6V. It became possible to compute the orbit owing to the observations at SOAR and by Horch et al. (2002, 2008).

11525-1408 = HDS $1676=$ HIP 57894. This binary has completed more than one revolution since its first resolution by Hipparcos. The coverage remains poor, however (exclusively at SOAR). The mass sum matches spectral type G0V.

$12485-1543=$ WSI $74 \mathrm{Aa}, \mathrm{Ab}=$ HIP 62505 $=$ HD 111312 is a K2.5V star GJ 1165 within $25 \mathrm{pc}$ of the Sun. Raghavan et al. (2010) state that it is a double-lined spectroscopic binary with a period of $2.698 \mathrm{yr}$, but do not give its orbital elements. Radial velocities of both components kindly provided by D. Latham were included in the combined orbital solution. We publish here only the "visual" elements and defer further analysis to another paper, as in the case of FIN 376.

$17066+0039=$ TOK $52 \mathrm{Ba}, \mathrm{Bb}=$ HIP 83716 was discovered at SOAR in 2009 (cf. TMH10), its 


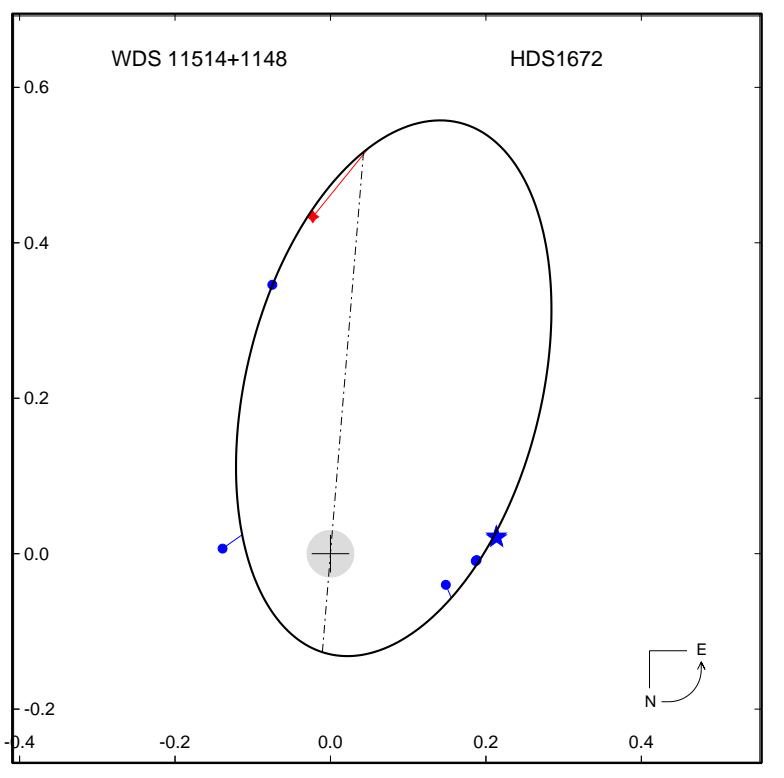

Fig. 10.- Orbit of HDS 1672

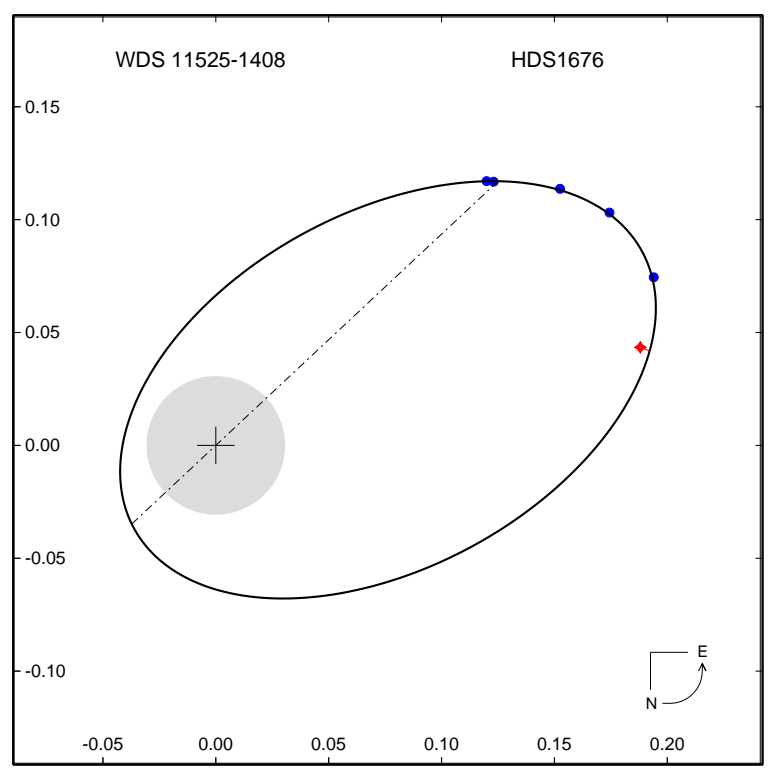

Fig. 11.- Orbit of HDS 1676

first orbit is computed here with almost a full revolution covered. The outer pair BU 823AB also has a computed orbit with a period of $532 \mathrm{yr}$, which may be nearly co-planar with that of $\mathrm{Ba}, \mathrm{Bb}$. The mass sum of $\mathrm{Ba}, \mathrm{Bb}$ estimated from its luminosity is $1.5 \mathcal{M}_{\odot}$ and matches well the dynamical mass inferred from the orbit.

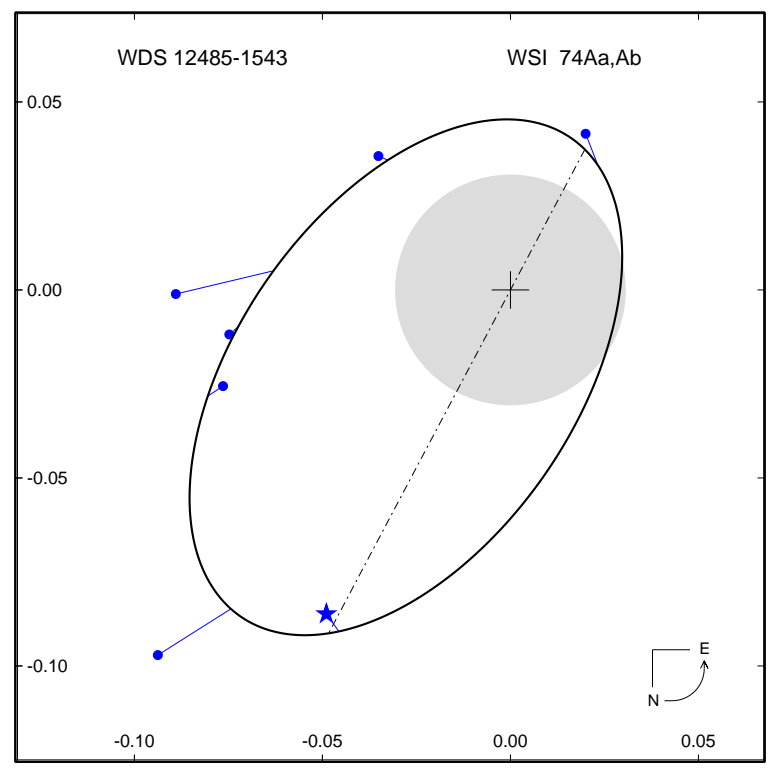

Fig. 12.- Orbit of WSI $74 \mathrm{Aa}, \mathrm{Ab}$.

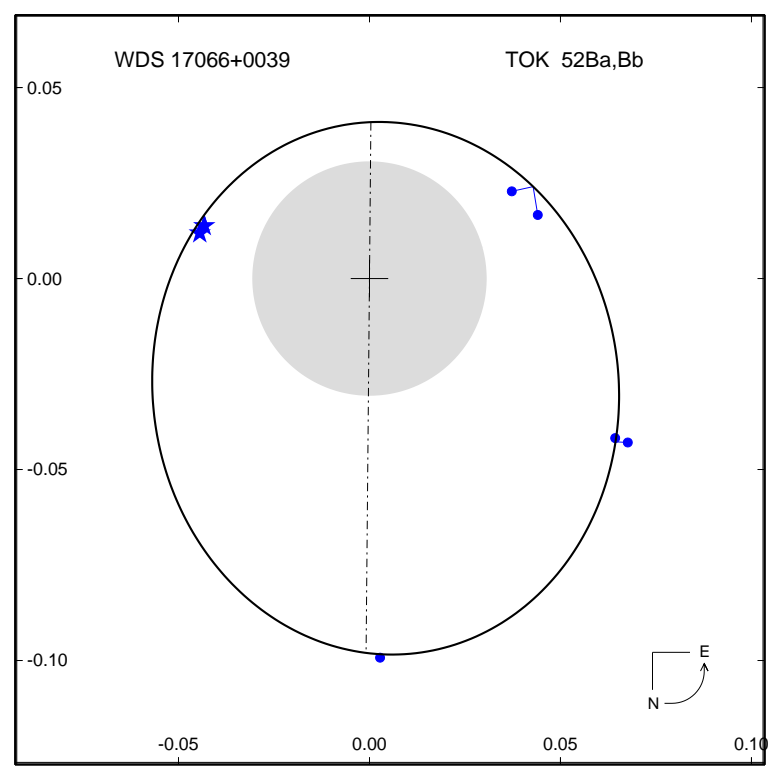

Fig. 13.- Orbit of TOK $52 \mathrm{Ba}, \mathrm{Bb}$

\section{Revised orbits}

Some pairs show substantial deviations from the published orbits, prompting their revisions. In several instances those revisions are only minor, as the orbit was already well constrained by the existing data. These revisions simply reduce errors of the elements or correct systematics such as scale 


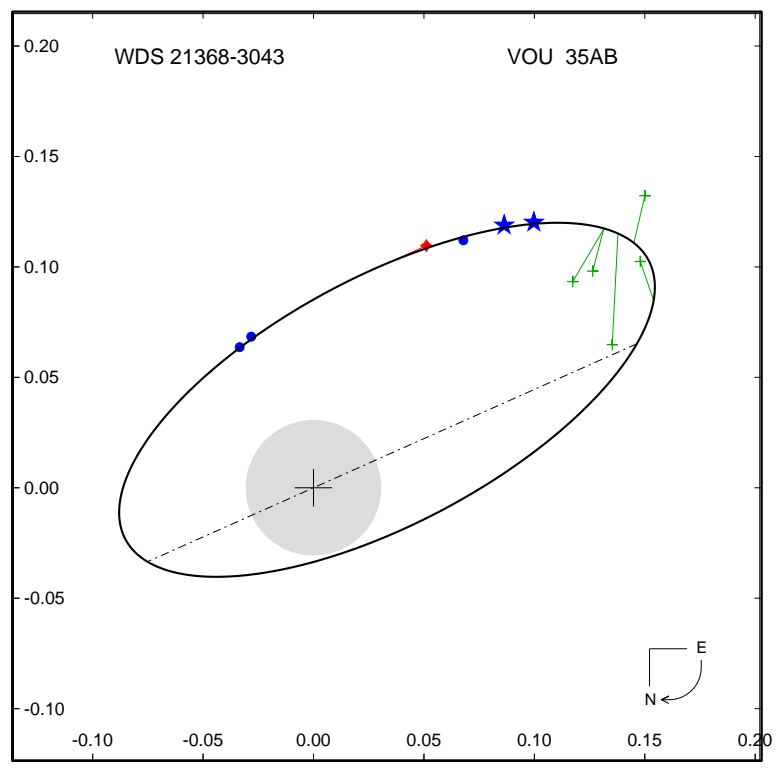

Fig. 14.- Orbit of VOU 35AB.

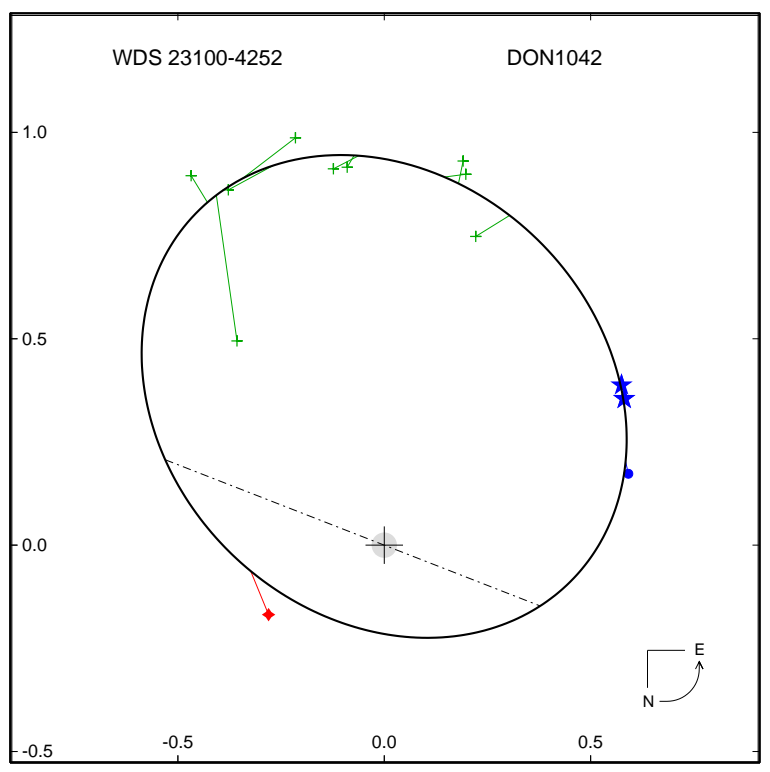

Fig. 15.- Orbit of DON 1042.

of the orbit, see e.g. Figure 16 .

The orbits of many long-period binaries are not yet fully covered by the observations; decades or even centuries of additional data are needed to do so. Here, the revision just improves the description of the orbital motion observed so far, while the period and semi-major axis remain essentially unconstrained, as illustrated in Figure 17. These should provide reliable ephemerides over the next several decades.

Substantial or drastic revisions of existing orbits are not uncommon. This happens when orbits were computed prematurely with insufficient or inaccurate observations. In such cases, the revised orbit had to be calculated from scratch. Hopefully, these new orbits are closer to the true ones and will be corrected incrementally in the future. The mass sum computed from the new orbits and known parallaxes is not substantially different from its estimate based on spectral type or luminosity of the stars. See Figure 18,

Table 7 lists elements of 45 orbits revised here. For orbits of grades $1-3$, the errors of each element are given, while the still preliminary orbits of grades 4 and 5 are given without formal errors. The last column of Table 7 contains references to the previous orbital solutions. Considering the availability of orbital plots in the VB6 on-line catalog, we do not provide them here, except three illustrative cases in Figures 16, 18.

The 25-yr astrometric sub-system belonging to the A-component of the bright visual binary $\zeta$ Aqr (WDS J22288-0001) was first resolved at SOAR and is listed in the WDS as EBE 1. Its orbit is still very preliminary, with period and eccentricity fixed. This mutiple system was recently discussed by Hartkopf et al. (2012).

We thank the operators of SOAR D. Maturana, S. Pizarro, P. Ugarte, A. Pastén for their help with labor-intensive speckle observations, and the anonymous referee for careful reading of the manuscript and tables. This work used the SIMBAD service operated by Centre des Données Stellaires (Strasbourg, France), bibliographic references from the Astrophysics Data System maintained by SAO/NASA, and the Washington Double Star Catalog maintained at USNO.

\section{Facilities: SOAR.}

\section{REFERENCES}

Alzner, A., Argyle, R. \& Anton, R. 2009, Inf. Circ., 169

Argyle, R. W. \& Alzner, A. 2010, Inf. Circ., 170

Balega, I. I., Balega, Yu. Yu., Hofmann, K.-H. et al. 1999, SvAL, 25, 797 


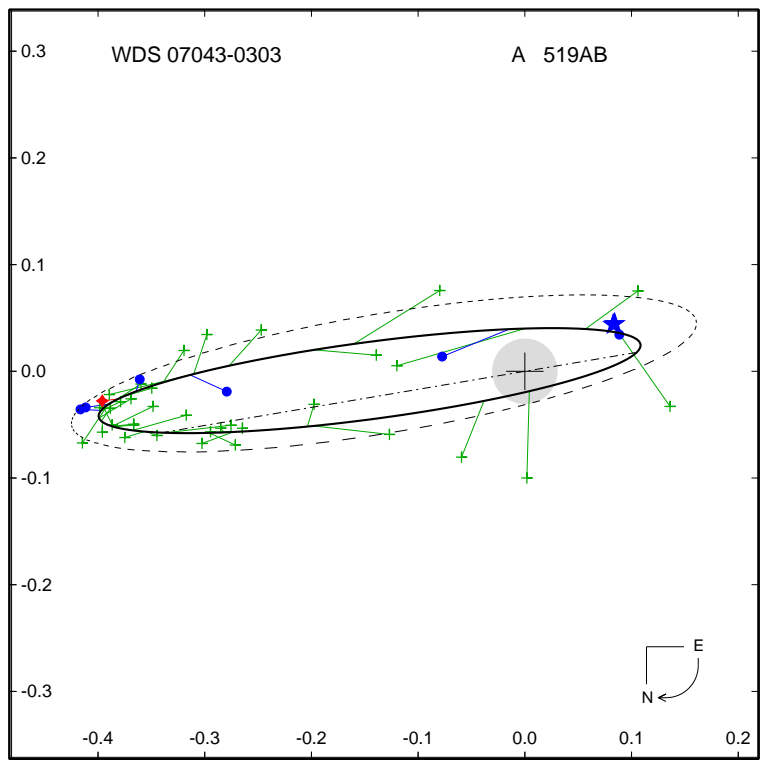

Fig. 16. - Minor revision of the orbit of A 519 (WDS J07043-0303), illustrating the change of scale in comparison with the previous orbit by Docobo \& Ling (2009) (dashed line).

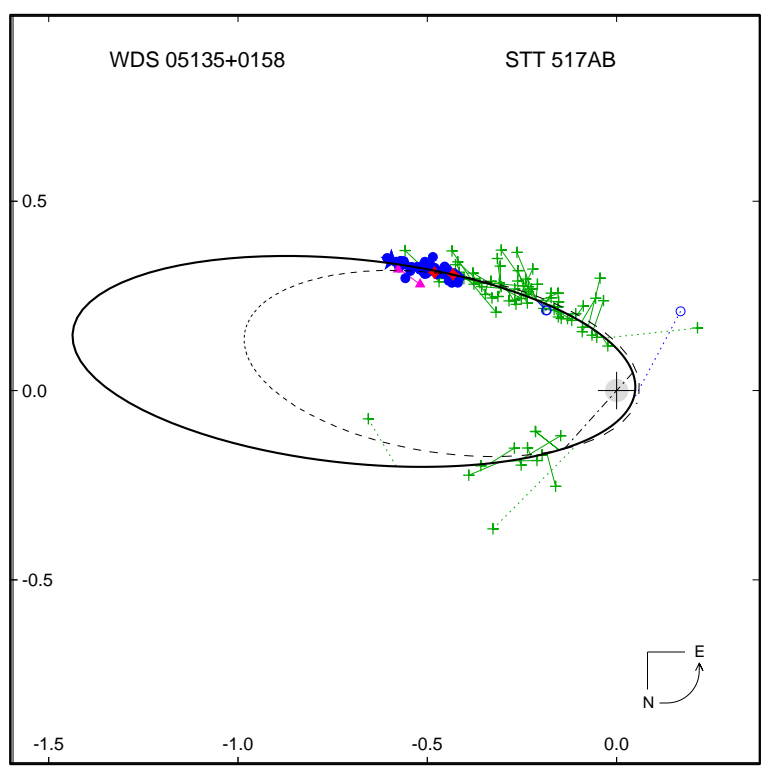

Fig. 17.- Orbit of STT $517 \mathrm{AB}, P=992 \mathrm{yr}$. The actual period can be much longer (few thousand years), it is not yet constrained by the observed arc.

Balega, I. I., Balega, Y. Y., Hofmann, K.-H. et al. 2005, A\&A, 433, 591

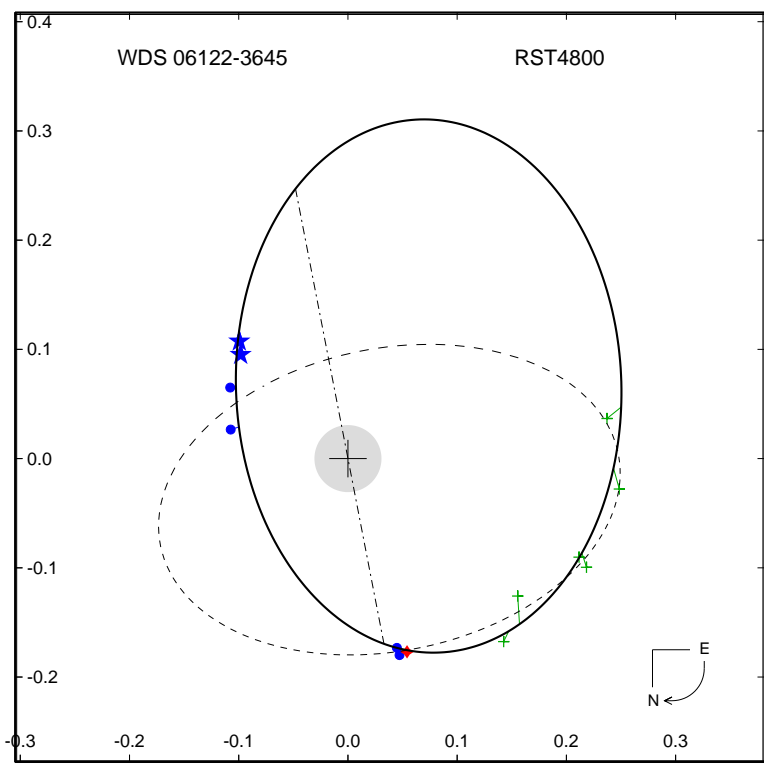

Fig. 18.- Orbit of RST 4800, $P=174 \mathrm{yr}$. New observations at SOAR cause drastic revision of the previous orbit by Seymour et al. (2002). The new orbit is still lacking coverage and will be further corrected in the coming decades.

Baize, P. 1986, A\&AS, 65, 551

Bender, C. F. \& Simon, M. 2008, ApJ, 689, 416

Beuzit, J.-L., Segransan, D., Forveille, T. et al. 2004, A\&A, 425, 997

van den Bos, W. H. 1961, Union Obs. Johannesburg Circ., 120, 380

Burnham, S.W. 1913, Carnegie Inst. Wash., 168

Cvetkovic, Z., Novakovic, B. \& Todorovic, N. 2008, New Astronomy, 13, 125

Cvetkovic, Z. 2008, AJ, 136, 1746

Cvetkovic, Z. \& Ninkovic, S. 2010, AN, 331, 304

Cvetkovic, Z. 2011, AJ, 141, 116

Cvetkovic, Z. 2012, Inf. Circ., 177

Davis, J., Mendez, A., Seneta, E. et al. 2005, MNRAS, 356, 1362

DeRosa, R. J., Patience, J., Vigan, A. et al. 2012, MNRAS, 422, 2765 
Docobo, J., Ling, J. \& Prieto, C. 19994, ApJS, 91, 793

Docobo, J. A. \& Tamazian, V. S. 2007, Inf. Circ., 162

Docobo, J. A. \& Ling, J. F. 2008, Inf. Circ., 164

Docobo, J. A. \& Ling, J. F. 2009, AJ, 138, 1159

Docobo, J. A. \& Ling, J. F. 2010, Inf. Circ., 172

Docobo, J. A. \& Ling, J. F. 2011, Inf. Circ., 174

Docobo, J. A. \& Campo, P. 2011, Inf. Circ., 174

Docobo, J. A. \& Campo, P. 2012, Inf. Circ., 176

Docobo, J. A. \& Andrade, M. 2013, Inf. Circ., 179

Dommanget, J. 1979, Bull. R. Astron. Obs. Belgium, 9,116

Ehrenreich, D., Lagrange, A.-M., Montagnier, G. et al. 2010, A\&A, 523, 73

Erceg, V. 1985, Bull. Obs. Astron. Belgrade, 135, 45

Finsen, W. S. 1964, Republic Obs. Circ., 123, 59

Forveille, T., Beuzit, J.-L., Delfosse, X. et al. 1999, A\&A, 351, 619

Griffin, R. F. 2012, JAA, 33, 29

Halbwachs, J.-L., Arenou, F., Mayor, M. et al. 2000, A\&A, 305, 581

Hartkopf, W. I., Mason, B. D. \& McAlister, H. A. 1996, AJ, 111, 370

Hartkopf, W. I., McAlister, H. A. \& Mason, B .D. 2001, AJ 122, 3480

Hartkopf, W. I., Mason, B. D., McAlister, H. A. et al. 2000, AJ, 119, 3084

Hartkopf, W. I. 2000, Inf. Circ., 141

Hartkopf, W. I. \& Mason, B. D. 2000, Inf. Circ., 142

Hartkopf, W. I., Mason, B. D. \& Worley, C. E. 2001, AJ, 122, 3472 (VB6)

Hartkopf, W. I. \& Mason, B. D. 2001a, Inf. Circ., 143
Hartkopf, W. I. \& Mason, B. D. 2001b, Inf. Circ., 145

Hartkopf, W. I. \& Mason, B. D. 2010, Inf. Circ., 170

Hartkopf, W. I. \& Mason, B. D. 2011, Inf. Circ., 175

Hartkopf, W. I., Tokovinin, A. \& Mason, B. D. 2012, AJ, 143, 42

Hartkopf, W. I. \& Harshaw, R. 2013a, Inf. Circ., 179

Hartkopf, W. I. \& Harshaw, R. 2013b, Inf. Circ. , 181

Heintz, W. D. 1978a, ApJS, 37, 71

Heintz, W. D. 1978b, ApJS, 37, 515

Heintz, W. D. 1981, ApJS, 45, 559

Heintz, W. D. 1984, A\&AS, 56, 5

Heintz, W. D. 1986a, A\&AS, 64, 1

Heintz, W. D. 1986b, A\&AS, 65, 411

Heintz, W. D. 1988, A\&AS, 72, 543

Heintz, W. D. 1990, A\&AS, 82, 65

Heintz, W. D. 1991, A\&AS, 90, 311

Heintz, W. D. 1993, A\&AS, 98, 209

Heintz, W. D. 1995, A\&AS, 99, 693

Heintz, W. D. 1996, AJ 111, 412

Heintz, W. D. 1997, ApJS, 111, 335

Heintz, W. D. \& Borgman, E. R. 1984, AJ, 89, 1068

Heintz, W. D. 1984, ApJ, 284, 806

Horch, E. P., Robinson, S. E., Meyer, R. D. et al. 2002, AJ, 123, 3442

Horch, E. P. Davidson, J. W., Jr., van Altena, W. F. et al 2006, AJ, 131, 1000

Horch, E. P., van Altena, W. F., Cyr, W. M., Jr. et al. 2008, AJ, 136, 312 
Jasinta, D. M. D. 1997, Visual Double Stars, ed. Docobo et al., 367

Ling, J. F. 2010, AJ, 139, 1521

Makarov, V. V. \& Kaplan, G. H., 2005, AJ, 129, $2420(\mathrm{MK} 05)$

Mante, R. 2001, Inf. Circ., 145

Mante, R. 2003, Inf. Circ., 150

Mason, B. D. \& Hartkopf, W. I. 1999, Inf. Circ., 138

Mason, B. D. \& Hartkopf, W. I. 2001, Inf. Circ., 144

Mason, B. D., Wycoff, G. L., Hartkopf, W. I. et al. 2001, AJ, 122, 3466 (WDS)

Mason, B. D., Hartkopf, W. I., Wycoff, G. L. et al. 2004, AJ, 127, 539

Mason, B. D., Hartkopf, W. I., Wycoff, G. L. \& Holdenried, E. R. 2006, AJ, 132, 2219

Mason, B. D. \& Hartkopf, W. I. 2005, Inf. Circ., 156

Mason, B. D., Hartkopf, W. I., Gies, D. R. et al. 2009, AJ, 137, 3358

Mason, B. D. \& Hartkopf, W. I. 2010, Inf. Circ., 170

Mason, B. D., Hartkopf, W. I. \& Tokovinin, A. 2010, AJ, 140, 735

Mason, B. D. \& Hartkopf, W. I. 2011a, Inf. Circ., 173

Mason, B. D. 2011, Inf. Circ., 173

Mason, B. D. \& Hartkopf, W. I. 2011b, Inf. Circ., 174

Mason, B. D. \& Hartkopf, W. I. 2012, Inf. Circ., 178

Muller, P. 1955, J. Obs., 38, 17

Muterspaugh, M. W., Hartkopf, W. I., Lane. B. F. et al. 2010, AJ, 140, 1623

Nordström, B., Mayor, M., Andersen, J. et al. 2004, A\&A, 418, 989
Norro, A. 1983, Bull. R. Astron. Obs. Belgium 9, 262

Olevic, D. \& Jovanovic, P. 2001, Serbian AJ, 163, 5

Olevic, D. \& Cvetkovic, Z. 2004a, Inf. Circ., 152

Olevic, D. \& Cvetkovic, Z. 2004b, A\&A, 415, 259

Olevic, D. \& Cvetkovic, Z. 2005, RevMexA\&A, 41, 17

Popovic, G. M. 1969, Bull. Obs. Astron. Belgrade, 27, 1, 33

Popovic, G. M. 1978, Bull. Obs. Astron. Belgrade, 129,9

Raghavan, D., McAlister, H. A., Henry, T. J. et al. 2010, ApJS, 190, 1

Reffert, S. \& Quirrenbach, A. 2011, A\&A, 527, 140

Riddle, R., Tokovinin, A., Mason, B. et al., 2014, ApJ, in preparation.

Rica Romero, F. M. 2010, Inf. Circ., 171

Rica Romero, F. M. \& Zirm, H. 2011, Inf. Circ., 174

Rica, F. M. 2012, JDSO, 8, 127

Rica Romero, F. M. 2012, Inf. Circ., 171

Rica Romero, F. M. 2013, Inf. Circ., 181

Scardia, M. 1983, Inf. Circ., 90

Scardia, M., Prieur, J.-L., Aristidi, E. \& Koechlin, L. 2000, AN, 321, 255

Scardia, M. 2001, Inf. Circ., 144

Scardia, M., Argyle, R. W., Prieur, J.-L. et al. 2007, AN, 328, 146

Scardia, M., Prieur, J.-L., Pansecchi, L. \& Argyle, R. W. 2008, Inf. Circ., 165

Scardia, M., Prieur, J.-L., Pansecchi, L. \& Argyle, R. W. 2010, Inf. Circ., 170

Seymour, D. \& Hartkopf, W. 1999, Inf. Circ., 139

Seymour, D., Mason, B. D., Hartkopf, W. I. \& Wycoff, G. L. 2002, AJ, 123, 1023 
Söderhjelm, S. 1999, A\&A, 341, 121

Starikova, G. A. 1981, SvAL, 7, 130

Tokovinin, A. A. 1999, SvAL, 25, 669

Tokovinin, A. A., Kiyaeva, O., Sterzik, M. et al. 2005, A\&A, 441, 695

Tokovinin A., Tighe R., Schurter P. et al. 2008, Proc. SPIE, 7015, 157

Tokovinin, A. \& Cantarutti, R. 2008, PASP, 120, 170

Tokovinin, A. 2012, AJ, 144, 56

Tokovinin, A. 2013, AJ, 145, 76

Tokovinin, A., Mason, B. D., \& Hartkopf, W. I. 2010, AJ, 139, 743 (TMH10)

Tokovinin, A. Cantarutti, R., Tighe, R. et al. 2010, PASP, 122, 1483

Tokovinin, A., Hartung, M., Hayward, Th. L., \& Makarov, V. V. 2012, AJ, 144, 7

Tokovinin, A. \& Lépine, S. 2012, AJ, 144, 102

Tokovinin, A., Hartung, M., \& Hayward, Th. L., 2013, AJ, 146, 8

Torres, C. A. O., Quast, G. R., Da Silva, L. et al. 2006, A\&A, 460, 695

Turner, N. H., ten Brummelaar, T. A., Roberts, L. C. et al. 2008, AJ, 136, 554

Wilson, R. H. 1976, MNRAS, 174, 75P

Zirm, H. 2007, Inf. Circ., 161

Zirm, H. 2008, Inf. Circ., 166

Zirm, H. 2010a, Inf. Circ., 170

Zirm, H. 2010b, Inf. Circ., 172

Zirm, H. 2013, Inf. Circ., 179

This 2-column preprint was prepared with the AAS LATEX macros $\mathrm{v} 5.2$. 
TABLE 2

MEASUREMENTS OF BINARY STARS AT SOAR

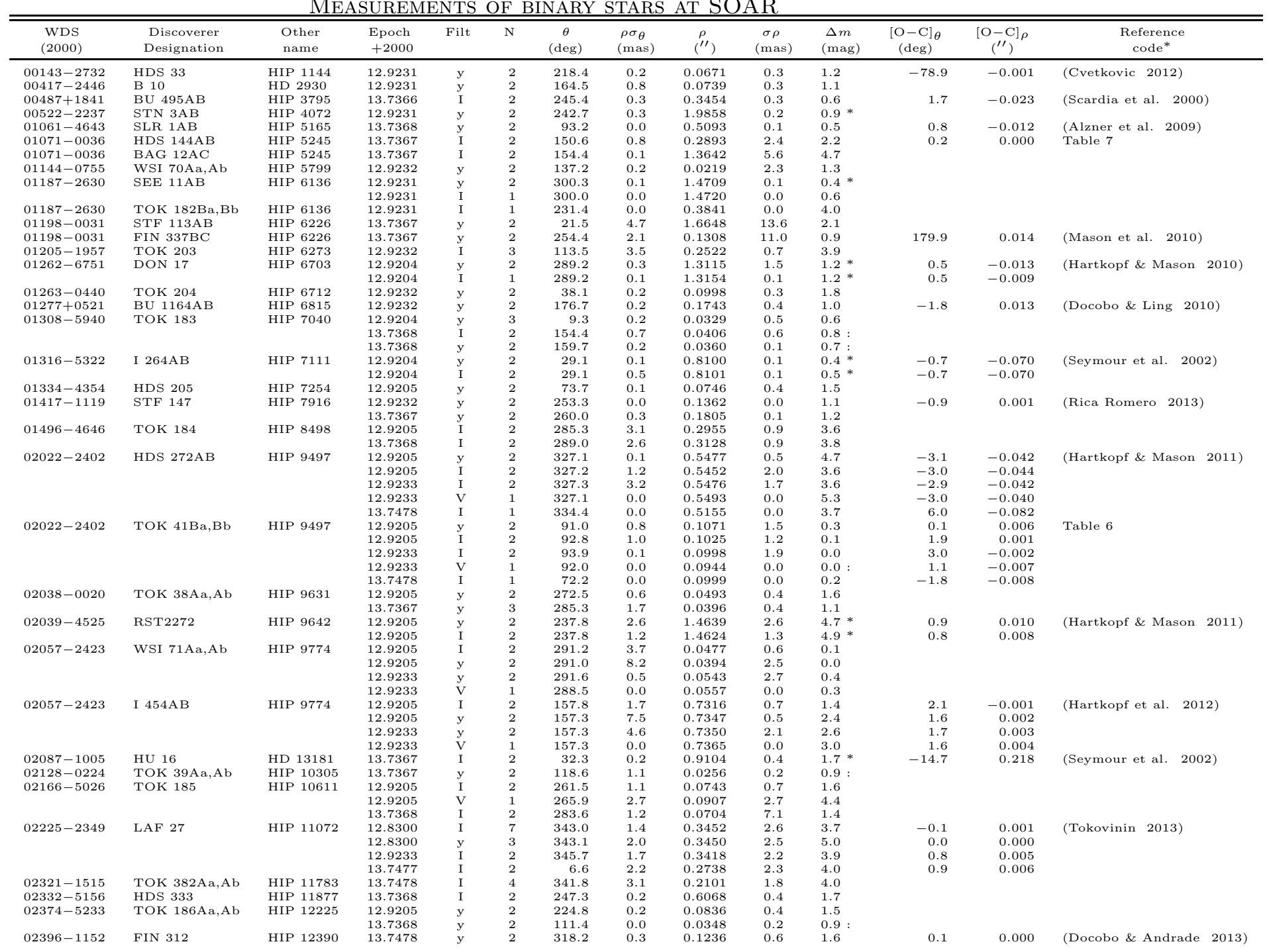


TABLE 2-Continued

\begin{tabular}{|c|c|c|c|c|c|c|c|c|c|c|c|c|c|}
\hline $\begin{array}{l}\text { WDS } \\
(2000)\end{array}$ & $\begin{array}{c}\text { Discoverer } \\
\text { Designation }\end{array}$ & $\begin{array}{l}\text { Other } \\
\text { name }\end{array}$ & $\begin{array}{c}\text { Epoch } \\
+2000 \\
\end{array}$ & Filt & $\mathrm{N}$ & $\begin{array}{c}\theta \\
(\mathrm{deg})\end{array}$ & $\begin{array}{l}\rho \sigma_{\theta} \\
(\mathrm{mas})\end{array}$ & $\begin{array}{l}\rho \\
(\prime \prime) \\
(1)\end{array}$ & $\begin{array}{c}\sigma \rho \\
(\mathrm{mas})\end{array}$ & $\begin{array}{l}\Delta m \\
(\mathrm{mag})\end{array}$ & $\begin{array}{c}{[\mathrm{O}-\mathrm{C}]_{\theta}} \\
(\mathrm{deg})\end{array}$ & $\begin{array}{c}{[\mathrm{O}-\mathrm{C}]_{\rho}} \\
\left({ }^{\prime \prime}\right)\end{array}$ & $\begin{array}{c}\begin{array}{c}\text { Reference } \\
\text { code* }\end{array} \\
\end{array}$ \\
\hline $02415-7128$ & B 1923 & HIP 12548 & 12.9232 & y & 2 & 326.0 & 0.3 & 0.1476 & 0.5 & 1.2 & 1.8 & 0.001 & (Hartkopf et al. 2012) \\
\hline & & & 13.7366 & 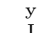 & 2 & 310.3 & 0.2 & 0.1445 & 0.2 & 1.5 & 1.5 & -0.001 & \\
\hline $02426-7947$ & TOK 362 & HIP 12654 & $\begin{array}{l}13.7366 \\
13.7366\end{array}$ & I & $\begin{array}{l}2 \\
2\end{array}$ & $\begin{array}{l}122.4 \\
122.6\end{array}$ & 0.8 & $\begin{array}{l}0.6270 \\
0.6200\end{array}$ & 0.8 & 2.6 & & & \\
\hline $02434-3756$ & TOK 187 & HIP 12716 & $\begin{array}{l}13.7366 \\
12.9232\end{array}$ & y & $\begin{array}{l}2 \\
2\end{array}$ & $\begin{array}{l}122.6 \\
345.5\end{array}$ & $\begin{array}{l}2.7 \\
9.8\end{array}$ & $\begin{array}{l}0.6200 \\
0.3055\end{array}$ & $\begin{array}{l}7.6 \\
5.8\end{array}$ & $\begin{array}{l}4.2 \\
5.2\end{array}$ & & & \\
\hline & & $1115=1210$ & 12.9232 & I & 2 & 347.3 & $\begin{array}{l}3.0 \\
3.3\end{array}$ & 0.3104 & $\begin{array}{l}5.8 \\
0.7\end{array}$ & $\begin{array}{l}5.2 \\
3.5\end{array}$ & & & \\
\hline $02434-6643$ & FIN 333 & HIP 12717 & 13.7366 & $\mathrm{y}$ & 2 & 215.1 & 0.2 & 0.4777 & 0.5 & 0.7 & 0.4 & 0.003 & (Mason \& Hartkopf 2011a) \\
\hline \multirow{3}{*}{$\begin{array}{l}02442-2530 \\
02456-7114\end{array}$} & $\begin{array}{l}\text { FIN } 379 \mathrm{Aa}, \mathrm{Ab} \\
\text { HDS } 357\end{array}$ & $\begin{array}{l}\text { HIP } 12780 \\
\text { HIP } 12884\end{array}$ & $\begin{array}{l}13.7477 \\
1.9232\end{array}$ & $\begin{array}{l}\mathrm{y} \\
\mathrm{y}\end{array}$ & $\begin{array}{c}2 \\
2\end{array}$ & $\begin{array}{r}223.0 \\
51.2\end{array}$ & $\begin{array}{l}0.1 \\
0.8\end{array}$ & $\begin{array}{l}0.1101 \\
0.3331\end{array}$ & $\begin{array}{l}0.2 \\
1.3\end{array}$ & $\begin{array}{l}0.9 \\
40\end{array}$ & $\begin{array}{l}2.4 \\
0.7\end{array}$ & $\begin{array}{l}-0.001 \\
-0.003\end{array}$ & (Hartkopf et al. 2012) \\
\hline & HDS 357 & HIP 12884 & $\begin{array}{l}12.9232 \\
12.9232\end{array}$ & $\begin{array}{l}\mathrm{y} \\
\mathrm{I}\end{array}$ & $\begin{array}{l}2 \\
1\end{array}$ & $\begin{array}{l}51.2 \\
51.3\end{array}$ & $\begin{array}{l}0.8 \\
0.6\end{array}$ & $\begin{array}{l}0.3331 \\
0.3364\end{array}$ & $\begin{array}{l}1.3 \\
0.6\end{array}$ & $\begin{array}{l}4.0 \\
3.5\end{array}$ & $\begin{array}{l}0.7 \\
0.8\end{array}$ & $\begin{array}{r}-0.003 \\
0.000\end{array}$ & (Hartkopf \& Mason 2011) \\
\hline & & & 13.7366 & y & 2 & 47.9 & 1.1 & 0.3400 & 0.7 & 3.9 & 1.2 & -0.001 & \\
\hline $02460-0457$ & $\begin{array}{l}\text { BU } 83 \\
\text { STF } 315\end{array}$ & HIP 12912 & 12.9205 & I & & 14.2 & & 0.9714 & 0.4 & $1.8 *$ & 1.5 & -0.003 & \\
\hline $\begin{array}{l}02493-1033 \\
02572-2458\end{array}$ & $\begin{array}{l}\mathrm{STF} 315 \\
\mathrm{BU} 741 \mathrm{AB}\end{array}$ & $\begin{array}{l}\text { HIP } 13168 \\
\text { HIP } 13772\end{array}$ & $\begin{array}{l}12.9205 \\
13.7478\end{array}$ & $\begin{array}{l}\mathrm{y} \\
\mathrm{y}\end{array}$ & $\begin{array}{l}2 \\
3\end{array}$ & $\begin{array}{l}165.1 \\
345.6\end{array}$ & $\begin{array}{l}0.2 \\
0.2\end{array}$ & $\begin{array}{l}1.3277 \\
0.7539\end{array}$ & $\begin{array}{l}0.2 \\
0.2\end{array}$ & $\begin{array}{l}1.1 \% \\
1.0\end{array}$ & 0.5 & 0.029 & \\
\hline $02572-2458$ & $\mathrm{BEU} 4 \mathrm{Ca}, \mathrm{Cb}$ & HIP 13769 & 13.7477 & $\begin{array}{l}\mathrm{y} \\
\mathrm{y}\end{array}$ & $\begin{array}{l}3 \\
2\end{array}$ & $\begin{array}{r}345.6 \\
33.6\end{array}$ & $\begin{array}{l}0.2 \\
0.8\end{array}$ & $\begin{array}{l}0.7539 \\
0.0893\end{array}$ & $\begin{array}{l}0.2 \\
1.1\end{array}$ & $\begin{array}{l}1.0 \\
1.5\end{array}$ & 0.5 & 0.029 & Rica 2012) \\
\hline \multirow{2}{*}{$03035+2304$} & HDS 389AB & HIP 14230 & 12.9232 & y & 2 & 359.9 & 0.4 & 0.1405 & & 1.7 & -0.6 & 0.004 & (Balega et al. 2005) \\
\hline & & & 12.9232 & $\mathrm{I}$ & 1 & 359.4 & 0.2 & 0.1403 & 0.2 & 1.3 & -1.0 & 0.004 & \\
\hline \multirow{2}{*}{$03167-0332$} & TOK $189 \mathrm{Aa}, \mathrm{Ab}$ & HIP 15247 & 12.9205 & I & 2 & 121.2 & 0.9 & 0.8672 & 0.9 & $3.6 *$ & & & \\
\hline & & & 12.9205 & $\mathrm{y}$ & 1 & 121.2 & 5.7 & 0.8760 & 5.7 & $5.6 *{ }^{*}>2>0$ & & & \\
\hline \multirow{2}{*}{$\begin{array}{l}03244-1539 \\
03307-1926\end{array}$} & A $2909 \mathrm{AB}$ & HIP 15868 & 13.7367 & y & 2 & 140.2 & 0.8 & 0.0320 & 0.3 & 0.6 & 35.1 & -0.072 & (Muller 1955) \\
\hline & HDS 441 & HIP 16348 & $\begin{array}{l}12.9233 \\
13.7367\end{array}$ & $\mathrm{y}$ & 2 & 181.1 & 0.2 & $\begin{array}{l}0.1769 \\
0.1572\end{array}$ & 0.1 & 0.4 & & & \\
\hline $03309-6200$ & TOK 190 & HIP 16370 & 13.7368 & y & $\frac{2}{2}$ & $\begin{array}{l}17.2 \\
228.4\end{array}$ & $\begin{array}{l}0.1 \\
0.3\end{array}$ & 0.0312 & $\begin{array}{l}0.2 \\
0.2\end{array}$ & $\begin{array}{l}0.6 \\
1.0\end{array}$ & & & \\
\hline $03339-3105$ & B 52 & HIP 16628 & 12.9233 & y & 2 & 170.8 & 0.0 & 0.0415 & 0.8 & 1.3 & 66.0 & 0.007 & (Heintz 1996) \\
\hline $03496-0220$ & YR 23 & HIP 17895 & 12.9206 & $\mathrm{y}$ & 3 & 311.4 & 0.1 & 0.3431 & 0.1 & 0.7 & & & 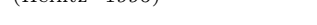 \\
\hline \multirow{3}{*}{$04007+2023$} & FIN $344 \mathrm{AB}$ & HIP 18262 & 12.9233 & $\mathrm{y}$ & 2 & 94.3 & 0.3 & 0.0868 & 0.5 & 0.8 & 1.2 & 0.002 & (Hartkopf et al. 2012) \\
\hline & TOK $363 \mathrm{Aa}, \mathrm{Ab}$ & HIP 18719 & $\begin{array}{r}13.7369 \\
13\end{array}$ & I & 1 & 94.2 & 1.3 & 0.0976 & 1.3 & 3.6 & & & \\
\hline & & & 13.7369 & y & 1 & 95.7 & 4.9 & 0.1023 & 4.9 & 5.2 & & & \\
\hline $04008+0505$ & A 1937 & HIP 18734 & 12.9234 & $\mathrm{v}$ & 3 & 173.8 & 1.3 & 0.0403 & 0.6 & 0.1 & 11.5 & 0.004 & Table 6 \\
\hline & & HLP 18839 & $\begin{array}{r}13.7369 \\
13.7367\end{array}$ & I & $\begin{array}{l}3 \\
2\end{array}$ & $\begin{array}{r}192.9 \\
1366\end{array}$ & 1.2 & $\begin{array}{l}0.0483 \\
0.4463\end{array}$ & 1.0 & 0.9 & -0.7 & 0.018 & (Mason \& Hartkonf 2011b) \\
\hline $\begin{array}{l}04024-2832 \\
04070-1000\end{array}$ & $\begin{array}{l}\text { DAW } 79 \\
\text { HDS } 521 \mathrm{AB}\end{array}$ & $\begin{array}{l}\text { HIP } 18839 \\
\text { HIP } 19206\end{array}$ & $\begin{array}{l}13.7367 \\
13.7369\end{array}$ & I & $\frac{2}{2}$ & $\begin{array}{l}1366.6 \\
276.9\end{array}-\mathrm{l}$ & $\begin{array}{l}0.1 \\
0.2\end{array}$ & $\begin{array}{l}0.4463 \\
0.1701\end{array}$ & $\begin{array}{l}0.2 \\
0.1\end{array}$ & $\begin{array}{l}1.1 \\
1.2\end{array}$ & $\begin{array}{l}-0.8 \\
-0.0\end{array}$ & $\begin{array}{l}0.005 \\
-0.002\end{array}$ & (Mason \& Hartkopf 2011b) \\
\hline $04107-0452$ & A 2801 & HIP 19508 & 13.7369 & y & 2 & 146.4 & 0.2 & 0.0386 & 0.1 & 0.7 & -11.4 & 0.008 & Table 7 \\
\hline $04119+2338$ & CHR 14 & HIP 19591 & 13.7369 & I & 2 & 21.0 & 0.5 & 0.2431 & 1.1 & 1.5 & -2.5 & 0.005 & (Mason \& Hartkopf 2010) \\
\hline $\begin{array}{l}04130-2832 \\
04136+0743\end{array}$ & $\begin{array}{l}\text { HWE } 10 \\
\text { A } 1938\end{array}$ & $\begin{array}{l}\text { HIP } 19684 \\
\text { HIP } 19719\end{array}$ & $\begin{array}{l}13.7367 \\
13.1309\end{array}$ & $\mathrm{y}$ & $\begin{array}{r}2 \\
2\end{array}$ & $\begin{array}{r}54.4 \\
152.0\end{array}$ & $\begin{array}{l}0.2 \\
0.1\end{array}$ & $\begin{array}{l}1.4130 \\
0.1036\end{array}$ & $\begin{array}{l}0.3 \\
0.2\end{array}$ & $\begin{array}{l}0.5{ }^{*} \\
0.9\end{array}$ & -0.4 & & (Muterspaugh et al. 2010) \\
\hline \multirow{2}{*}{$04142-4608$} & $\begin{array}{l}\text { A } 195838 \\
\text { RST2338 }\end{array}$ & HIP 19758 & $\begin{array}{l}13.1308 \\
13.1282\end{array}$ & I & $\frac{2}{2}$ & $\begin{array}{l}152.0 \\
319.8\end{array}$ & $\begin{array}{l}.1 \\
0.1\end{array}$ & $\begin{array}{l}0.1068 \\
0.2998\end{array}$ & $\begin{array}{l}0.2 \\
0.3\end{array}$ & $\begin{array}{l}0.9 \\
1.2\end{array}$ & $\begin{array}{r}-0.4 \\
1.7\end{array}$ & $\begin{array}{l}0.014 \\
0.014\end{array}$ & (Mouterhjaugh 1999) \\
\hline & & & $\begin{array}{l}13.1282 \\
12.9234\end{array}$ & y & 2 & $\begin{array}{l}319.8 \\
1110\end{array}$ & $\begin{array}{l}0.1 \\
10\end{array}$ & $\begin{array}{l}0.2995 \\
0.1206\end{array}$ & 0.5 & & 1.7 & 0.013 & \\
\hline $04163+0710$ & WSI 97 & HIP 19911 & $\begin{array}{l}12.9234 \\
12.9234\end{array}$ & $\begin{array}{l}\mathrm{I} \\
\mathrm{y}\end{array}$ & $\begin{array}{c}2 \\
2\end{array}$ & $\begin{array}{l}111.0 \\
111.2\end{array}$ & $\begin{array}{l}1.0 \\
1.1\end{array}$ & 0.1206 & $\begin{array}{l}0.7 \\
0.8\end{array}$ & $\begin{array}{l}1.6 \\
20\end{array}$ & & & \\
\hline $04205-0119$ & RST4769 & HIP 20257 & $\begin{array}{l}12.9234 \\
12.9233\end{array}$ & $\begin{array}{l}\mathrm{y} \\
\mathrm{y}\end{array}$ & $\frac{2}{2}$ & $\begin{array}{r}111.2 \\
26.7\end{array}$ & $\begin{array}{l}1.1 \\
0.1\end{array}$ & $\begin{array}{l}0.1201 \\
0.2091\end{array}$ & $\begin{array}{l}0.8 \\
0.1\end{array}$ & $\begin{array}{l}2.0 \\
0.3\end{array}$ & 0.9 & -0.000 & (Hartkopf et al. 2012) \\
\hline $04215-2055$ & B $1935 \mathrm{AB}$ & HIP 20342 & 13.7367 & I & 2 & 99.6 & 0.3 & 0.3372 & 0.4 & 1.3 & 0.0 & 0.002 & Table 6 \\
\hline & HU 304 & HIP 20522 & $\begin{array}{l}13.7367 \\
13.1310\end{array}$ & $\mathrm{y}$ & 2 & $\begin{array}{l}99.6 \\
26.4\end{array}$ & 0.4 & 0.3374 & 0.4 & 2.1 & $\begin{array}{r}0.0 \\
-0.7\end{array}$ & $\begin{array}{r}0.002 \\
-0.005\end{array}$ & (Hartkonf 2000) \\
\hline \multirow{2}{*}{ 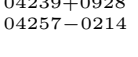 } & $\begin{array}{l}\text { HU } 304 \\
\text { BU } 403\end{array}$ & $\begin{array}{l}\text { HIP } 20522 \\
\text { HIP } 20673\end{array}$ & $\begin{array}{l}13.1310 \\
12.9234\end{array}$ & y & $\begin{array}{l}2 \\
2\end{array}$ & 84.1 & $\begin{array}{l}0.0 \\
0.4\end{array}$ & $\begin{array}{l}0.2876 \\
0.9722\end{array}$ & 0.2 & $\begin{array}{l}0.3 \\
1.5 *\end{array}$ & -0.7 & -0.005 & (Hartkopf 2000) \\
\hline & & & 12.9234 & I & 1 & 84.1 & 0.3 & 0.9723 & 0.3 & $1.5 *$ & & & \\
\hline \multirow{2}{*}{$\begin{array}{l}04258+1800 \\
04311-4522\end{array}$} & COU2682 & HIP 20679 & & I & 2 & 347.4 & 0.2 & 0.2331 & 0 & 1.1 & 0.9 & 0.001 & Table 7 \\
\hline & TOK 208 & HIP 21079 & $\begin{array}{l}13.1282 \\
13.1282\end{array}$ & $\mathrm{l}$ & ${ }_{2}^{2}$ & $\begin{array}{l}23.7 \\
23.9\end{array}$ & $\begin{array}{l}0.7 \\
9.4\end{array}$ & $\begin{array}{l}1.6604 \\
1.6691\end{array}$ & $\begin{array}{l}0.7 \\
9.4\end{array}$ & $\begin{array}{l}3.5^{*} \\
4.6 *\end{array}$ & & & \\
\hline $04340-5503$ & B $2092 \mathrm{AB}$ & HIP 212 & & & 2 & 131.1 & 0.1 & 0.2490 & 0.2 & 1.0 & 0.1 & 0.014 & (Hartkopf \& Mason 2010) \\
\hline $04362+0814$ & A $1840 \mathrm{AB}$ & HIP 21434 & 13.1310 & I & 2 & & 0.3 & 0.1666 & 1. & 0.5 & 0.3 & 0.011 & \\
\hline \multirow[t]{2}{*}{$04368-1733$} & A 2915 & BD-17 917 & $\begin{array}{l}12.92 \\
12.92\end{array}-\mathrm{r} \mathrm{r} \mathrm{r}$ & $\mathrm{I}_{\mathrm{y}}^{\mathrm{y}}$ & ${ }_{2}^{2}$ & $\begin{array}{l}80.8 \\
79.4\end{array}$ & $\begin{array}{l}0.4 \\
0.9\end{array}$ & $\begin{array}{l}0.0776 \\
0.0848\end{array}$ & $\begin{array}{l}0 . \\
0 .\end{array}-1$ & $\begin{array}{l}1.6 \\
1.1\end{array}$ & $\begin{array}{l}79.7 \\
78.3\end{array}$ & $\begin{array}{l}-0.165 \\
-0.158\end{array}$ & (Baize 1986) \\
\hline & & & 13.73 & $\begin{array}{l}1 \\
\mathrm{I}\end{array}$ & ${ }_{2}^{2}$ & $\begin{array}{l}9.4 \\
98.3\end{array}$ & $\begin{array}{l}0.9 \\
0.4\end{array}$ & 0.0944 & 0. & 1.3 & 95.5 & $\begin{array}{l}-0.158 \\
-0.145\end{array}$ & \\
\hline $04395-4507$ & I 1489 & HIP 21698 & $\begin{array}{l}13.1282 \\
13.1282\end{array}$ & y & 1 & 210.1 & 0.3 & 0.0 & 0. & 1.1 & 6.8 & 0.012 & (Docoo \& Campo 2012) \\
\hline \multirow{3}{*}{$04422+0259$} & & & 13.1282 & I & 3 & 208.0 & 0.3 & 0.0543 & 2 . & & 4.8 & 0.017 & \\
\hline & A 2424 & HIP 21880 & 13.1310 & $\begin{array}{l}\mathrm{V} \\
\mathrm{I}\end{array}$ & 2 & $\begin{array}{l}36.8 \\
33.5\end{array}$ & 1.8 & 0.0373 & 0.2 & 0.0 & -9.2 & -0.130 & (Wilson 1976) \\
\hline & & & $\begin{array}{l}13.1310 \\
13.7369\end{array}$ & $\begin{array}{l}\mathrm{I} \\
\mathrm{y}\end{array}$ & $\frac{1}{2}$ & $\begin{array}{l}33.5 \\
40.8\end{array}$ & $\begin{array}{l}0.1 \\
0.1\end{array}$ & $\begin{array}{l}0.0447 \\
0.0589\end{array}$ & $\begin{array}{l}0.1 \\
0.1\end{array}$ & $\begin{array}{l}0.2 \\
0.7\end{array}$ & $\begin{array}{r}-12.5 \\
-4.8\end{array}$ & $\begin{array}{l}-0.122 \\
-0.106\end{array}$ & \\
\hline $04496+0212$ & A 2621 & HIP 22428 & 13.7 & I & 2 & 139.9 & 0.1 & 7 & 0.6 & 0. & 4.2 & 0.005 & ymour $\&$ Ha \\
\hline $04590-1623$ & $\mathrm{BU} 314 \mathrm{AB}$ & HIP 23166 & 12.9233 & y & 2 & 319.9 & 0.2 & 0.8453 & 0.2 & 1.7 & 1.5 & 0.051 & ö́derhic \\
\hline & A $1844 \mathrm{AB}$ & HIP 23395 & 13.1 & $\mathrm{y}$ & 2 & 25.0 & 0.4 & 0.3423 & 0.4 & 2. & -0.7 & 0.008 & Mason \& Hartkopf \\
\hline $05059-1355$ & $\begin{array}{l}\text { A } 3009 \\
\end{array}$ & $\begin{array}{l}\text { HIP } 23716 \\
\text { HIP } 23818\end{array}$ & 12.9207 & I & 2 & $\begin{array}{r}271.5 \\
486\end{array}$ & 0.6 & 1.1856 & 0.3 & $1.4^{*}$ & $\begin{array}{r}1.0 \\
-0.9\end{array}$ & 0.009 & Tablc \\
\hline
\end{tabular}


TABLE 2-Continued

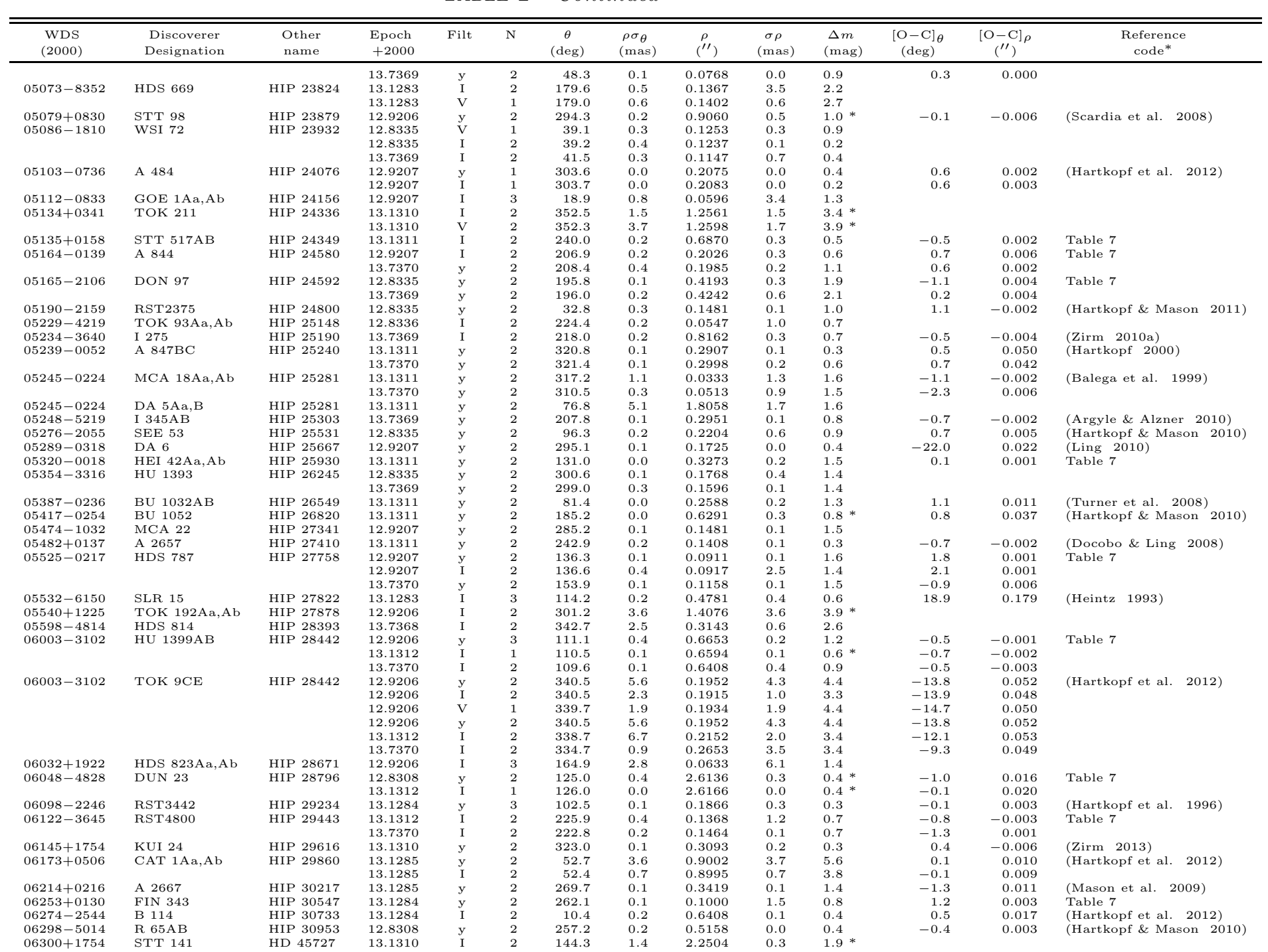


TABLE 2-Continued

\begin{tabular}{|c|c|c|c|c|c|c|c|c|c|c|c|c|c|}
\hline $\begin{array}{l}\text { WDS } \\
(2000)\end{array}$ & $\begin{array}{c}\text { Discoverer } \\
\text { Designation } \\
\end{array}$ & $\begin{array}{l}\text { Other } \\
\text { name }\end{array}$ & $\begin{array}{l}\text { Epoch } \\
+2000 \\
\end{array}$ & Filt & $\mathrm{N}$ & $\begin{array}{c}\theta \\
(\mathrm{deg})\end{array}$ & $\begin{array}{l}\rho \sigma_{\theta} \\
(\mathrm{mas})\end{array}$ & $\begin{array}{l}\rho \\
\left(^{\prime \prime}\right) \\
\end{array}$ & $\begin{array}{c}\sigma \rho \\
(\mathrm{mas})\end{array}$ & $\begin{array}{l}\Delta m \\
(\mathrm{mag})\end{array}$ & $\begin{array}{c}{[\mathrm{O}-\mathrm{C}]_{\theta}} \\
(\mathrm{deg})\end{array}$ & $\begin{array}{c}{[\mathrm{O}-\mathrm{C}]_{\rho}} \\
\left({ }^{\prime \prime}\right)\end{array}$ & $\begin{array}{c}\begin{array}{c}\text { Reference } \\
\text { code* }\end{array} \\
\end{array}$ \\
\hline $06314+0749$ & A 2817 & HIP 31089 & 13.1285 & I & 2 & 335.4 & 0.4 & 0.1222 & 0.8 & 0.5 & 23.1 & -0.006 & (Popovic 1969) \\
\hline $06359-3605$ & FIN $19 \mathrm{Aa}, \mathrm{Ab}$ & HIP 31509 & $\begin{array}{l}13.7370 \\
12.9206\end{array}$ & $\mathrm{y}$ & 2 & $\begin{array}{l}347.7 \\
348\end{array}$ & $\begin{array}{l}0.0 \\
0.0\end{array}$ & $\begin{array}{l}0.3137 \\
0.3022\end{array}$ & 0.0 & 1.2 & 1.4 & -0.000 & (Hartkopf \& Mason 2011) \\
\hline $06359-3605$ & RST $4816 \mathrm{Ba}, \mathrm{Bb}$ & HIP 31547 & $\begin{array}{l}12.9206 \\
13.7370\end{array}$ & $\begin{array}{l}\mathrm{y} \\
\mathrm{y}\end{array}$ & ${ }_{2}^{2}$ & $\begin{array}{l}348.9 \\
126.3\end{array}$ & $\begin{array}{l}0.0 \\
0.1\end{array}$ & $\begin{array}{l}0.3022 \\
0.2062\end{array}$ & $\begin{array}{l}0.1 \\
0.1\end{array}$ & $\begin{array}{l}1.2 \\
0.8\end{array}$ & $\begin{array}{r}0.7 \\
-0.2\end{array} \quad-2$ & $\begin{array}{l}-0.000 \\
-0.001\end{array}$ & (Tokovinin 2012) \\
\hline & & & 12.9206 & $\mathrm{y}$ & 2 & 131.3 & 0.1 & 0.1974 & 0.2 & 0.7 & -0.3 & -0.001 & \\
\hline $06380-6132$ & I $5 \mathrm{AB}$ & HIP 31711 & 12.8308 & y & 2 & 101.2 & 0.5 & 0.5654 & 0.5 & 2.4 & -0.7 & -0.003 & (Zirm 2010b) \\
\hline $\begin{array}{l}06410+0954 \\
06425-4234\end{array}$ & $\begin{array}{l}\text { CHR } 168 \mathrm{Aa}, \mathrm{Ab} \\
\text { I } 283\end{array}$ & HIP 31978 & $\begin{array}{l}13.1285 \\
13.1312\end{array}$ & $\mathrm{y}$ & ${ }_{2}^{2}$ & $\begin{array}{l}262.0 \\
193.6\end{array}$ & 0.2 & 0.1180 & 0.3 & 1.5 & -0.2 & 0.008 & (Cvetkovic 2011) \\
\hline $06425-4234$ & I 283 & HIP 32111 & $\begin{array}{l}13.1312 \\
13.7370\end{array}$ & $\begin{array}{l}\mathrm{I} \\
\mathrm{I}\end{array}$ & $\begin{array}{l}2 \\
2\end{array}$ & $\begin{array}{l}193.6 \\
194.1\end{array}$ & $\begin{array}{l}0.4 \\
1.3\end{array}$ & $\begin{array}{l}2.3519 \\
2.3518\end{array}$ & $\begin{array}{l}1.5 \\
1.0\end{array}$ & $\begin{array}{l}2.5 \text { * } \\
2.5 *\end{array}$ & & & \\
\hline $06439-5434$ & HDS 934 & HIP 32242 & 12.8308 & $\mathrm{y}$ & 2 & 102.5 & 0.3 & 0.1126 & 0.3 & $0.7:$ & -0.4 & 0.000 & (Hartkopf et al. 2012) \\
\hline $06454-3148$ & EHR $9 \mathrm{~A}$ Ba & HIP 32366 & $\begin{array}{l}13.7371 \\
13.7370\end{array}$ & I & 2 & $\begin{array}{l}140.9 \\
2066\end{array}$ & 0.3 & 0.1023 & 0.3 & 0.5 & -3.3 & 0.009 & \\
\hline $06454-3148$ & $\mathrm{EHR} 9 \mathrm{Ba}, \mathrm{Bb}$ & $\begin{array}{l}\text { HIP } 32366 \\
\text { HIP } 32366\end{array}$ & $\begin{array}{l}13.7370 \\
13.7370\end{array}$ & I & $\begin{array}{l}2 \\
2\end{array}$ & $\begin{array}{r}206.6 \\
74.5\end{array}$ & $\begin{array}{l}1.6 \\
2.2\end{array}$ & $\begin{array}{l}1.3649 \\
0.1012\end{array}$ & $\begin{array}{l}2.9 \\
9.8\end{array}$ & $\begin{array}{l}6.3 \\
1.0\end{array}$ & & & \\
\hline $06485-1226$ & $\begin{array}{l}\text { A } 2935 \\
\text { A } 2930 \text {, }\end{array}$ & $\begin{array}{l}\text { HD } 49572 \\
\text { HD }\end{array}$ & 13.1284 & I & 2 & 320.2 & 0.2 & 0.2135 & 0.8 & 0.3 & -2.0 & -0.006 & (Hartkopf et al. 2012) \\
\hline $06490-1509$ & $\mathrm{AC} 4$ & HIP 32677 & 13.1284 & y & 2 & 345.2 & 0.1 & 0.3842 & 0.2 & 1.8 & 10 & (50) & He \\
\hline $06493-0216$ & FIN 322 & HIP 32698 & $\begin{array}{l}13.1284 \\
13.7370\end{array}$ & y & 2 & 270.7 & 0.1 & 0.1044 & 0.4 & 0.9 & 1.0 & 0.002 & (Hartkopf \& Mason 2011) \\
\hline $\begin{array}{l}06494-3325 \\
06545-2734\end{array}$ & $\begin{array}{l}\text { RST22433 } \\
\text { B } 706\end{array}$ & $\begin{array}{l}\text { HIP } 32709 \\
\text { HIP } 33197\end{array}$ & $\begin{array}{l}13.7370 \\
13.1284\end{array}$ & $\begin{array}{l}\text { I } \\
\text { I }\end{array}$ & $\begin{array}{l}3 \\
2\end{array}$ & $\begin{array}{l}222.7 \\
292.4\end{array}$ & $\begin{array}{l}0.6 \\
0.2\end{array}$ & $\begin{array}{l}1.5160 \\
0.7229\end{array}$ & $\begin{array}{l}0.3 \\
0.2\end{array}$ & $\begin{array}{l}0.1 * \\
0.4\end{array}$ & 0.3 & -0.003 & Table \\
\hline $06573-3530$ & & HIP 33451 & 13.7371 & $\mathrm{y}$ & 2 & 146.5 & 0.0 & 0.2485 & 0.0 & 0.4 & 0.5 & 0.004 & (Docobo \& Ling 2009) \\
\hline $06573-4929$ & RST5253AB & HIP 33455 & $\begin{array}{l}13.1312 \\
13.7371\end{array}$ & I & 2 & $\begin{array}{l}315.4 \\
3165\end{array}$ & 3.4 & 0.1681 & 0.4 & 0.5 & 2.6 & 0.007 & Hartkopf et al. 2012) \\
\hline & & & $\begin{array}{l}13.7371 \\
13.1312\end{array}$ & $\begin{array}{l}\text { I } \\
\text { I }\end{array}$ & 2 & $\begin{array}{r}316.5 \\
2386\end{array}-10$ & $\begin{array}{l}0.8 \\
16\end{array}$ & $\begin{array}{l}0.1762 \\
1.0610\end{array}$ & $\begin{array}{l}2.3 \\
2.0\end{array}$ & $\begin{array}{l}0.8 \\
20\end{array}$ & 1.0 & 0.008 & \\
\hline $06573-4929$ & RST5253AC & HIP 33455 & $\begin{array}{l}13.1312 \\
13.7371\end{array}$ & $\begin{array}{l}\mathrm{I} \\
\mathrm{I}\end{array}$ & $\begin{array}{l}2 \\
2\end{array}$ & $\begin{array}{l}238.6 \\
239.2\end{array}$ & $\begin{array}{l}1.6 \\
1.8\end{array}$ & $\begin{array}{l}1.0660 \\
1.0685\end{array}$ & $\begin{array}{l}2.0 \\
0.5\end{array}$ & $\begin{array}{l}2.0 \\
2.3\end{array}$ & & & \\
\hline $07015-0942$ & A $3042 \mathrm{AB}$ & HD 52590 & 12.9208 & $\mathrm{y}$ & 2 & 232.7 & 0.2 & 0.1562 & 1.0 & 1.2 & 181.2 & -0.000 & Table 7 \\
\hline $07029-1313$ & HDS 980 & HIP 33973 & 13.1313 & $\mathrm{y}$ & 2 & 226.1 & 0.6 & 0.4723 & 0.6 & 3.2 & & & \\
\hline $07043-0303$ & A $519 \mathrm{AB}$ & HIP 34110 & $\begin{array}{l}12.9208 \\
12.9208\end{array}$ & I & 2 & $\begin{array}{l}117.9 \\
1178\end{array}$ & $\begin{array}{l}1.0 \\
0.8\end{array}$ & 0.0953 & 1.8 & 0.5 & 6.6 & -0.002 & Table 7 \\
\hline $07074-2350$ & CHR 246 & HIP 34360 & 12.9207 & $\mathrm{y}_{\mathrm{y}}$ & 2 & 111.8 & 0.7 & 0.0888 & $\begin{array}{l}1.4 \\
1.3\end{array}$ & $\begin{array}{l}0.6 \\
1.9\end{array}$ & 0.5 & -0.004 & \\
\hline $07092+1903$ & CHR 216 & HIP 34524 & 12.9210 & I & & 165.1 & 0.2 & 0.1150 & 1.7 & 1.4 & & & \\
\hline & & ПIIT 54024 & 12.9210 & & 2 & 164.5 & 0.6 & 0.1164 & 2.2 & 2.1 & & & \\
\hline $07113-1032$ & A 2122 & HIP 34702 & 13.1285 & I & 2 & 176.2 & 0.3 & 0.0848 & 0.4 & 0.5 & 24.4 & -0.039 & (Seymour et al. 2002) \\
\hline & & & 13.1285 & $\mathrm{~V}$ & 2 & 177.2 & 0.1 & 0.0841 & 0.1 & 0.4 & 25.4 & -0.039 & \\
\hline $07123-4030$ & HDS1001 & HIP 34802 & 12.8335 & $\mathrm{y}$ & 2 & 269.4 & 0.4 & 0.6797 & 0.4 & 4.2 & & & \\
\hline $07143-2621$ & FIN 323 & HIP & & $\mathrm{y}$ & 2 & & 0.0 & & 0.0 & 0.2 & 180.7 & -0.031 & Table 7 \\
\hline $07155-7552$ & I 312 & HIP 35102 & $\begin{array}{l}13.1311 \\
13.1311\end{array}$ & ${ }_{\mathrm{I}}^{\mathrm{y}}$ & $\begin{array}{l}2 \\
1\end{array}$ & $\begin{array}{l}197.7 \\
196.3\end{array}$ & $\begin{array}{l}0.2 \\
0.1\end{array}$ & $\begin{array}{l}0.0705 \\
0.0673\end{array}$ & $\begin{array}{l}0.6 \\
0.1\end{array}$ & $\begin{array}{l}0.8 \\
0.3\end{array}$ & & & \\
\hline $07168+0059$ & A 2855 & HIP 35221 & 13.1285 & I & 2 & 164.3 & 0.2 & 0.2861 & 0.2 & 0.4 & 2.2 & 0.010 & (Mason et al. 2009) \\
\hline $07171-1202$ & A $2123 \mathrm{AB}$ & HIP 35261 & 13.1285 & I & 2 & 356.1 & 0.2 & 0.3957 & 0.0 & 0.5 & 0.2 & 0.009 & (Mason \& Hartkopf 2011a) \\
\hline $\begin{array}{l}07175-4659 \\
07185-5721\end{array}$ & $\begin{array}{l}\text { I } 7 \\
\text { HDS } 1013 \mathrm{Aa}, \mathrm{Ab}\end{array}$ & $\begin{array}{l}\text { HIP } 35296 \\
\text { HIP } 35374\end{array}$ & 12.8335 & $\mathrm{y}$ & 2 & 202.7 & 0.2 & $\begin{array}{l}0.7149 \\
0.353\end{array}$ & 0.1 & 1.4 & -0.9 & $\begin{array}{r}0.006 \\
-0.003\end{array}$ & (Mason \& Hartkopf 2011b) \\
\hline $\begin{array}{l}07185-5721 \\
07185-5721\end{array}$ & $\begin{array}{l}\text { HDS1013Aa, Ab } \\
\text { RST 244Ba. Bb }\end{array}$ & $\begin{array}{l}\text { HIP } 35374 \\
\text { HIP } 35374\end{array}$ & 13.1311 & 1 & ${ }^{2}$ & 233.9 & 0.1 & 0.3532 & 0.1 & 0.5 & 0.2 & -0.003 & (Hartkopf et al. 2012) \\
\hline $0718-5721$ & $\begin{array}{l}\mathrm{R} \\
\mathrm{RST} 244 \mathrm{Ba}, \mathrm{Bb}\end{array}$ & $\begin{array}{l}\text { HIP } 353774 \\
\text { HIP }\end{array}$ & 13.1311 & 1 & 1 & 357.1 & 10. & 0.914 & 10. & 0.3 & & & \\
\hline $\begin{array}{l}07185-5721 \\
07187-2457\end{array}$ & $\begin{array}{ll}\text { RST } 2 \\
\text { RIN } 3\end{array}$ & $\begin{array}{l}\text { HIP } 35374 \\
\text { HIP } 35415\end{array}$ & $\begin{array}{l}13.1 \\
12.9\end{array}$ & 1 & 1 & 262.4 & 0.0 & 2.9035 & 0.0 & 3.6 & & & \\
\hline $07187-2457$ & $\begin{array}{l}\text { FOK } 42 \mathrm{Aa}, \mathrm{Ab} \\
\text { TOK }\end{array}$ & $\begin{array}{l}\text { HIP } 35415 \\
\text { HIP } 35415\end{array}$ & $\begin{array}{l}12.9207 \\
12.9207\end{array}$ & y & 2 & $\begin{array}{r}129 \\
87\end{array}$ & $\begin{array}{l}0.0 \\
0.6\end{array}$ & $\begin{array}{l}0.1 \\
0.9\end{array}-9$ & $\begin{array}{l}2.4 \\
2.1\end{array}$ & $\begin{array}{l}0.9 \\
4.4\end{array}$ & & & \\
\hline $07294-1500$ & STF $1104 \mathrm{AB}$ & HIP 36395 & 12.9208 & $\mathrm{y}$ & 1 & 34.6 & 0.1 & 1.8227 & 0.1 & $1.3 *$ & -0.4 & 0.004 & Table 7 \\
\hline $07324-3558$ & RST 4855 & HIP 36657 & 13.13 & $\mathrm{y}$ & 2 & 6.8 & 0.1 & 0.2720 & 0.0 & 1.5 & 0.1 & -0.026 & Table 7 \\
\hline $07374-3458$ & FIN $324 \mathrm{AC}$ & HIP 37096 & & $\mathrm{y}$ & 2 & 30 & 0.1 & & 0.1 & 1.5 & 0.6 & -0.001 & (Hartkopf et al. 2012) \\
\hline $07378-0236$ & A $534 \mathrm{AC}$ & HIP 37134 & $\begin{array}{l}13.1285 \\
13.1285\end{array}$ & $\begin{array}{l}\mathrm{y} \\
\mathrm{I}\end{array}$ & $\begin{array}{l}1 \\
2\end{array}$ & $\begin{array}{l}303.8 \\
303.7\end{array}$ & $\begin{array}{l}1.1 \\
0.8\end{array}$ & $\begin{array}{l}1.4389 \\
1.4361\end{array}$ & $\begin{array}{l}1.1 \\
1.4\end{array}$ & $\begin{array}{l}2.8 * \\
3.2 *\end{array}$ & & & \\
\hline $07411-0124$ & A 196 & HD 61809 & 12.9 & I & 2 & 309.2 & 0.1 & 0.3163 & 0.1 & 0.2 & 179.7 & -0.001 & Table 7 \\
\hline $07430-1704$ & $x=0-1$ & HIP 37608 & 13.1313 & $\mathrm{y}$ & 2 & & 0.1 & 0.4 & 0.1 & 0.7 & 0.2 & 0.005 & Table 7 \\
\hline $07435+0329$ & TOK $355 \mathrm{Aa}, \mathrm{Ab}$ & HIP 376 & $\begin{array}{l}13.1285 \\
13.1285\end{array}$ & $\stackrel{\mathrm{y}}{\mathrm{I}}$ & $\frac{2}{2}$ & $\begin{array}{l}330.2 \\
329.6\end{array}$ & $\begin{array}{l}1.7 \\
4.0\end{array}$ & $\begin{array}{l}0.2078 \\
0.2075\end{array}$ & $\begin{array}{l}5.2 \\
0.8\end{array}$ & $\begin{array}{l}3.7 \\
3.2\end{array}$ & & & \\
\hline $07435-2711$ & В 73 & HIP 3 & 13 & & 2 & 6.2 & 0.1 & 0.08 & 0.2 & 0.5 & & & \\
\hline $07448-3344$ & 9001900 & HIP 37781 & 13.1313 & I & 2 & 29.0 & 0.1 & 1.0 & 0.6 & $0.0 *$ & & & \\
\hline $07456-34$ & TOK $193 \mathrm{Aa}, \mathrm{Ab}$ & HIP 37853 & $\begin{array}{l}12.8335 \\
12.8335\end{array}$ & $\mathrm{y}_{\mathrm{I}}$ & $\begin{array}{l}2 \\
2\end{array}$ & $\begin{array}{l}95.5 \\
95.3\end{array}$ & $\begin{array}{l}0.5 \\
2.3\end{array}$ & $\begin{array}{l}0.2322 \\
0.2221\end{array}$ & $\begin{array}{l}0.9 \\
1.0\end{array}$ & $\begin{array}{l}4.0 \\
3.1\end{array}$ & & & \\
\hline $07478-0332$ & RST4375 & HIP 38039 & 13.1 & I & 2 & 278 & 0.3 & 0.2 & $\begin{array}{l}1.0 \\
0.8\end{array}$ & $\begin{array}{l}3.1 \\
0.7\end{array}$ & 0.2 & 0.001 & Table 6 \\
\hline & & & 13.1285 & $\mathrm{y}$ & 2 & 278.7 & 0.3 & 0.2139 & 0.5 & 1.0 & 0.4 & 0.002 & \\
\hline $07479-1212$ & STF 1146 & HIP 38048 & 13.1313 & $\mathrm{y}$ & 2 & 341.0 & 0.1 & 1.1226 & 0.3 & $1.8 *$ & -0.4 & -0.005 & (Hartkopf \& Harshaw 2013a) \\
\hline $07490-245$ & TOK 194 & HIP 38146 & 12.9208 & $\mathrm{y}$ & 2 & 162.3 & 0.2 & 0.0482 & 0.2 & 1.1 & & & \\
\hline $07508+0317$ & 2880 & HIP 38300 & $\begin{array}{l}13.1313 \\
13.1285\end{array}$ & $\mathrm{y}$ & ${ }_{2}^{2}$ & $\begin{array}{l}176.1 \\
152.9\end{array}$ & $\begin{array}{l}0.1 \\
0.1\end{array}$ & $\begin{array}{l}0.0471 \\
0.1503\end{array}$ & $\begin{array}{l}0.1 \\
0.1\end{array}$ & 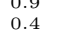 & -4.8 & 0.000 & (Hartkopf et al \\
\hline $07518-1354$ & BU 101 & HIP 38382 & 12.9208 & $\mathrm{y}$ & 2 & 290.3 & 0.3 & 0.5469 & 0.3 & 1.0 & 0.1 & 0.003 & (Tokovinin 2012 \\
\hline
\end{tabular}


TABLE 2-Continued

\begin{tabular}{|c|c|c|c|c|c|c|c|c|c|c|c|c|c|}
\hline $\begin{array}{l}\text { WDS } \\
(2000)\end{array}$ & $\begin{array}{c}\text { Discoverer } \\
\text { Designation }\end{array}$ & $\begin{array}{l}\text { Other } \\
\text { name }\end{array}$ & $\begin{array}{c}\text { Epoch } \\
+2000 \\
\end{array}$ & Filt & $\mathrm{N}$ & $\begin{array}{c}\theta \\
(\mathrm{deg})\end{array}$ & $\begin{array}{l}\rho \sigma_{\theta} \\
\text { (mas) }\end{array}$ & $\begin{array}{l}\rho \\
(\prime \prime)\end{array}$ & $\begin{array}{c}\sigma \rho \\
\text { (mas) }\end{array}$ & $\begin{array}{l}\Delta m \\
(\mathrm{mag})\end{array}$ & $\begin{array}{c}{[\mathrm{O}-\mathrm{C}]_{\theta}} \\
(\mathrm{deg})\end{array}$ & $\begin{array}{c}{[\mathrm{O}-\mathrm{C}]_{\rho}} \\
\left({ }^{\prime \prime}\right)\end{array}$ & $\begin{array}{c}\text { Reference } \\
\text { code* }\end{array}$ \\
\hline \multirow{4}{*}{$\begin{array}{l}07522-4035 \\
07523-2626\end{array}$} & TOK 195 & HIP 38414 & 12.8335 & & 2 & 108.5 & 0.9 & 0.0254 & 2.9 & $2.4:$ & & & \\
\hline & WSI $54 \mathrm{AB}$ & HIP 38430 & 12.9208 & $\mathrm{y}$ & 2 & & 2.2 & 0.0447 & 2.8 & 0.7 & & & \\
\hline & & & $\begin{array}{l}12.9208 \\
12.9208\end{array}$ & $\mathrm{~V}$ & ${ }_{2}^{1}$ & $\begin{array}{l}235.6 \\
235.0\end{array}$ & $\begin{array}{l}0.0 \\
0.8\end{array}$ & $\begin{array}{l}0.0466 \\
0.0427\end{array}$ & $\begin{array}{l}0.0 \\
6.5\end{array}$ & $\begin{array}{l}0.4 \\
0.1\end{array}$ & & & \\
\hline & & & $\begin{array}{l}12.9208 \\
13.1313\end{array}$ & $\begin{array}{l}\mathrm{R} \\
\mathrm{V}\end{array}$ & $\begin{array}{l}2 \\
3\end{array}$ & 234.5 & $\begin{array}{l}0.8 \\
1.0\end{array}$ & $\begin{array}{l}0.0427 \\
0.0455\end{array}$ & 6.5 & 0.1 & & & \\
\hline \multirow{3}{*}{$07523-2626$} & WSI $54 \mathrm{AC}$ & HIP 38430 & $\begin{array}{l}13.1313 \\
13.1313\end{array}$ & $\mathrm{~V}$ & 3 & 230.7 & 1.2 & $\begin{array}{l}0.0455 \\
0.0901\end{array}$ & $\begin{array}{l}7.6 \\
6.1\end{array}$ & $\begin{array}{l}1.2 \\
1.0\end{array}$ & & & \\
\hline & WOS S4AC & H115 30400 & 12.9208 & $\mathrm{y}$ & 2 & 228.9 & 5.6 & 0.0908 & $\begin{array}{l}6.1 \\
1.6\end{array}$ & $\begin{array}{l}1.0 \\
1.0\end{array}$ & & & \\
\hline & & & 12.9208 & $\mathrm{~V}$ & 1 & 232.8 & 0.0 & 0.0926 & 0.0 & 0.7 & & & \\
\hline & & & $\begin{array}{l}12.9208 \\
13.1313\end{array}$ & $\begin{array}{l}\mathrm{R} \\
\mathrm{y}\end{array}$ & $\begin{array}{l}2 \\
2\end{array}$ & $\begin{array}{l}230.6 \\
358.4\end{array}$ & $\begin{array}{l}1.6 \\
0.1\end{array}$ & $\begin{array}{l}0.0915 \\
0.3865\end{array}$ & $\begin{array}{l}7.2 \\
0.2\end{array}$ & 0.1 & & & \\
\hline $\begin{array}{l}07528-0526 \\
07546-0248\end{array}$ & $\begin{array}{l}\text { FIN } 325 \\
\text { STF1157 }\end{array}$ & $\begin{array}{l}\text { HIP } 38474 \\
\text { HD } 64607\end{array}$ & $\begin{array}{l}13.1313 \\
13.1313\end{array}$ & $\begin{array}{l}\mathrm{y} \\
\mathrm{y}\end{array}$ & $\begin{array}{l}2 \\
2\end{array}$ & $\begin{array}{l}358.4 \\
177.0\end{array}$ & $\begin{array}{l}0.1 \\
0.1\end{array}$ & $\begin{array}{l}0.3865 \\
0.6753\end{array}$ & $\begin{array}{l}0.2 \\
0.2\end{array}$ & $\begin{array}{l}1.2 \\
0.4\end{array}$ & $\begin{array}{l}-0.7 \\
-1.8\end{array}$ & $\begin{array}{r}0.002 \\
-0.018\end{array}$ & Hartkopf et al. 1996) \\
\hline $08024+0409$ & $\begin{array}{l}\text { STF1175 } \\
\text { STF1157 }\end{array}$ & HIP 39325 & 12.9210 & I & 2 & 285.1 & 0.1 & 1.4298 & 0.9 & $1.3 *$ & $\begin{array}{l}-1.8 \\
-4.3\end{array}$ & $\begin{array}{r}-0.050 \\
0.050\end{array}$ & Olevic \& Jovanovic 2001) \\
\hline $08125-4616$ & $\mathrm{CHR} 143 \mathrm{Aa}, \mathrm{Ab}$ & HIP 40183 & 12.9208 & $\mathrm{y}$ & 2 & 350.4 & 0.1 & 0.0632 & 0.6 & 0.8 & -6.0 & 0.033 & Mason et al. 2010) \\
\hline $\begin{array}{l}08125-4616 \\
08151-0655\end{array}$ & $\begin{array}{l}\text { SEE 96AB } \\
\text { MET } 52\end{array}$ & $\begin{array}{l}\text { HIP } 40183 \\
\text { HIP } 40419\end{array}$ & $\begin{array}{l}12.9208 \\
12.9209\end{array}$ & $\mathrm{y}$ & ${ }^{2}$ & $\begin{array}{r}274.9 \\
96.1\end{array}$ & $\begin{array}{l}0.7 \\
5.9\end{array}$ & $\begin{array}{l}0.6275 \\
1.4716\end{array}$ & $\begin{array}{c}1.8 \\
10.7\end{array}$ & $1.0 *$ & & & \\
\hline $\begin{array}{l}081151-0655 \\
08173-0522\end{array}$ & $\begin{array}{l}\text { MET } 52 \\
\text { A } 337\end{array}$ & $\begin{array}{l}\text { HIP } 40419 \\
\text { HIP } 40592\end{array}$ & $\begin{array}{l}12.9209 \\
13.1285\end{array}$ & $\begin{array}{l}\mathrm{I} \\
\mathrm{y}\end{array}$ & $\begin{array}{l}2 \\
2\end{array}$ & $\begin{array}{l}96.1 \\
62.1\end{array}$ & $\begin{array}{l}5.9 \\
0.6\end{array}$ & $\begin{array}{l}1.4716 \\
0.5293\end{array}$ & $\begin{array}{l}10.7 \\
0.4\end{array}$ & $\begin{array}{l}5.7{ }^{*} \\
0.9:\end{array}$ & -0.9 & 0.001 & Table 7 \\
\hline $08213-0136$ & STF1216 & HIP 40941 & 13.1285 & y & 2 & 304.8 & 0.2 & 0.5312 & 0.1 & 0.7 & -0.8 & 0.009 & Table 7 \\
\hline $08250-4246$ & $\mathrm{CHR} 226 \mathrm{Aa}, \mathrm{Ab}$ & & 12.9209 & y & & 264.6 & 0.7 & 0.0451 & 0.8 & & & & \\
\hline $08250-4246$ & & HIP 41250 & 12.9209 & y & 2 & 103.4 & 0.1 & 0.5369 & 0.1 & 0.8 & & & \\
\hline $08251-4910$ & RST 321 & HIP 41261 & 13.1287 & $\mathrm{y}$ & 2 & 167.0 & 0.1 & 0.3325 & 0.1 & 0.8 & -0.1 & 0.012 & (Hartkopf et al. 2012) \\
\hline \multirow[t]{2}{*}{$08270-5242$} & В 1606 & HIP 41426 & 12.9208 & y & 2 & 324.8 & 0.2 & 0.0649 & 0.2 & 1.0 & 2.1 & -0.007 & Tokovinin 2012 \\
\hline & & & $\begin{array}{l}13.1287 \\
12.9208\end{array}$ & $\mathrm{y}$ & $\frac{1}{2}$ & 337.9 & 0.1 & 0.0599 & 0.1 & 1.0 & 4.0 & -0.008 & \\
\hline $\begin{array}{l}08275-5501 \\
08276-2051\end{array}$ & $\begin{array}{l}\text { FIN } 116 \\
\text { B } 2179\end{array}$ & $\begin{array}{l}\text { HIP } 41464 \\
\text { HIP } 41475\end{array}$ & $\begin{array}{l}12.9208 \\
13.1314\end{array}$ & $\mathrm{y}$ & $\begin{array}{r}2 \\
2\end{array}$ & $\begin{array}{l}233.1 \\
220.2\end{array}$ & $\begin{array}{l}0.0 \\
0.1\end{array}$ & $\begin{array}{l}0.2511 \\
0.4364\end{array}$ & $\begin{array}{l}0.0 \\
0.1\end{array}$ & $\begin{array}{l}0.4 \\
1.8\end{array}$ & 0.6 & 0.002 & Table 7 \\
\hline $08280-3507$ & FIN $314 \mathrm{Aa}, \mathrm{Ab}$ & HIP 41515 & $\begin{array}{l}13.1314 \\
12.9209\end{array}$ & $\begin{array}{l}\mathrm{y} \\
\mathrm{y}\end{array}$ & $\begin{array}{l}2 \\
2\end{array}$ & $\begin{array}{r}220.2 \\
46.5\end{array}$ & $\begin{array}{l}0.1 \\
0.2\end{array}$ & $\begin{array}{l}0.4364 \\
0.1164\end{array}$ & $\begin{array}{l}0.1 \\
0.2\end{array}$ & $\begin{array}{l}1.8 \\
1.1\end{array}$ & -5.2 & -0.006 & (Hartkopf et al. 2012) \\
\hline $08289-1552$ & RST 4403 & HIP 41609 & $\begin{array}{l}12.9209 \\
12.9209\end{array}$ & $\begin{array}{l}\mathrm{y} \\
\mathrm{I}\end{array}$ & $\begin{array}{l}2 \\
1\end{array}$ & $\begin{array}{l}264.1 \\
264.0\end{array}$ & $\begin{array}{l}7.2 \\
0.3\end{array}$ & $\begin{array}{l}1.3236 \\
1.3205\end{array}$ & $\begin{array}{l}2.2 \\
0.3\end{array}$ & $\begin{array}{l}3.5 * \\
2.7\end{array}$ & & & \\
\hline $08291-4756$ & FIN $315 \mathrm{Aa}, \mathrm{Ab}$ & HIP 41616 & 13.1287 & $\mathrm{y}$ & 2 & 205.6 & 0.1 & 0.1028 & 0.0 & 1.5 & 0.6 & 0.000 & (Docobo \& Andrade 2013) \\
\hline $08315-1935$ & & HIP 41817 & 13.1314 & $\mathrm{y}$ & 2 & 290.4 & 0.0 & 0.3626 & 0.0 & 0.9 & 1.0 & -0.002 & (Mason et al. 2009) \\
\hline $08317-2601$ & I 807 & HD 72397 & 13.1314 & y & 2 & 161.5 & 0.0 & 0.4367 & 0.2 & 0.4 & & & \\
\hline $08327-5528$ & $\mathrm{HDS} 1221 \mathrm{Aa}, \mathrm{Ab}$ & HIP 41906 & 13.1287 & y & 2 & 42.8 & 0.8 & 0.0479 & 0.4 & 2.0 & & & \\
\hline $08331-2436$ & BU 205AB & HIP 41949 & 13.1314 & $\mathrm{y}$ & 2 & 291.0 & 0.1 & 0.5614 & 0.2 & 0.3 & 2.2 & -0.028 & (Hartkopf \& Mason 2010) \\
\hline \multirow{3}{*}{$08380-0844$} & FIN 335 & HIP 42075 & 13.1314 & & 2 & 245.5 & 0.2 & 0.0762 & 0.0 & 0.5 & & -0.007 & \\
\hline & HDS1242 & HIP 42345 & 12.9209 & I & 2 & 56.0 & 0.3 & 0.1560 & 0.1 & 1.2 & -2.4 & 0.022 & Hartkopf et al. 2012) \\
\hline & & & $\begin{array}{r}12.9209 \\
131314\end{array}$ & y & 2 & 56.2 & 0.2 & 0.1549 & 0.3 & $1.4 *$ & -2.2 & 0.021 & \\
\hline \multirow{3}{*}{$08391-5557$} & BU $208 \mathrm{AB}$ & HIP 42430 & 13.1314 & $\mathrm{y}$ & 2 & 40.4 & 0.0 & 0.9771 & 0.2 & $1.3 *$ & -6.0 & 0.189 & (Heintz 1990) \\
\hline & $\mathrm{HU} 1443 \mathrm{AB}$ & HIP 42424 & 13.1287 & $\begin{array}{l}\mathrm{y} \\
\mathrm{y}\end{array}$ & ${ }^{2}$ & 18.2 & 2.8 & 0.9179 & 0.9 & 1.6 & & & \\
\hline & & & 13.1287 & I & & 18.5 & 3.8 & 0.9173 & 1.8 & 1.4 & & & \\
\hline $08391-5557$ & TOK $356 \mathrm{BC}$ & HIP 42424 & 13.1287 & $\mathrm{y}$ & 2 & 129.4 & 3.8 & 0.0861 & 4.1 & 2.7 & & & \\
\hline & & & 13.1287 & $\mathrm{I}$ & & 126.3 & 7.1 & 0.0743 & 6.6 & 2.8 & & & \\
\hline $\begin{array}{l}08421-5245 \\
08431-1225\end{array}$ & $\begin{array}{l}\text { B } 1624 \\
\text { RST3603 }\end{array}$ & $\begin{array}{l}\text { HIP } 42695 \\
\text { HIP } 42790\end{array}$ & $\begin{array}{l}12.9208 \\
12.9209\end{array}$ & $\stackrel{\mathrm{y}}{\mathrm{I}}$ & $\begin{array}{r}2 \\
2\end{array}$ & $\begin{array}{l}230.3 \\
325.5\end{array}$ & $\begin{array}{l}0.2 \\
0.1\end{array}$ & 0.2286 & $\begin{array}{l}0.2 \\
0.1\end{array}$ & $\begin{array}{l}1.5 \\
0.4\end{array}$ & $\begin{array}{r}-0.3 \\
3.2\end{array}$ & $\begin{array}{l}0.014 \\
0.003\end{array}$ & (Hartkopf et al. 2012) \\
\hline $\begin{array}{l}08447-4238 \\
0845\end{array}$ & $\begin{array}{l}\text { RST3603 } \\
\text { CHR 238 }\end{array}$ & HIP 42916 & 12.9209 & y & $\frac{2}{2}$ & $\begin{array}{l}320.5 \\
295.7\end{array}$ & $\begin{array}{l}0.1 \\
0.2\end{array}$ & 0.0640 & $\begin{array}{l}0.1 \\
0.1\end{array}$ & $\begin{array}{l}0.4 \\
2.1\end{array}$ & $\begin{array}{l}3.2 \\
2.5\end{array}$ & $\begin{array}{l}0.007 \\
-0.007\end{array}$ & (Hartkopt et al. 2012) \\
\hline $08447-5443$ & I $10 \mathrm{AB}$ & HIP 42913 & 13.1287 & y & & 262.9 & 0.4 & 0.3650 & 0.4 & 3.3 & -0.9 & -0.018 & (Mason 2011) \\
\hline $08474-1703$ & BU 586 & HIP 43152 & 13.1314 & $\mathrm{y}$ & 2 & 44.3 & 0.1 & 0.2242 & 0.1 & 1.3 & 6.1 & -0.133 & (Mason et al. \\
\hline $08486+0237$ & A 2551 & HD 75192 & 13.1286 & $\mathrm{v}$ & 2 & 100.7 & 1.1 & 0.1309 & 1.1 & 1.7 & 0.3 & 0.008 & (Hartkopf et al. 2012) \\
\hline $08494+0341$ & A 2899 & HIP 43304 & 13.1286 & I & 2 & 239.2 & 1.4 & 0.6555 & 1.4 & $2.6 *$ & & & \\
\hline $08495+0852$ & $\mathrm{BU} 1068 \mathrm{AB}$ & HIP 43317 & 12.9210 & I & 2 & 321.1 & 0.3 & 0.3763 & 0.2 & 1.4 & 0.2 & 0.006 & (Hartkopf \& Mason 2010) \\
\hline \multirow{2}{*}{$08538-4731$} & FIN 316 & HIP 43671 & 13.1287 & y & 2 & 103.2 & 0.0 & 0.0813 & 0.2 & 0.7 & 1.8 & -0.001 & (Hartkopf et al. 2012) \\
\hline & & HIP 43 & $\begin{array}{l}13.1287 \\
13.1286\end{array}$ & $\begin{array}{l}1 \\
\mathrm{y}\end{array}$ & & $\begin{array}{l}104.4 \\
258.3\end{array}$ & $\begin{array}{l}0.2 \\
0.5\end{array}$ & $\begin{array}{l}0.0794 \\
0.2142\end{array}$ & $\begin{array}{l}0.1 \\
0.3\end{array}$ & $\begin{array}{l}0.6 \\
2.3\end{array}$ & $\begin{array}{r}2.9 \\
11.4\end{array}$ & $\begin{array}{l}-0.002 \\
-0.019\end{array}$ & (Zirm 2007) \\
\hline $\begin{array}{l}08539+0149 \\
08542-0229\end{array}$ & $\begin{array}{l}\text { A } 2554 \\
\text { A } 1754\end{array}$ & $\begin{array}{l}\text { HIP } 43676 \\
\text { HD } 76119\end{array}$ & $\begin{array}{l}13.1286 \\
12.9210\end{array}$ & $\begin{array}{l}\mathrm{y} \\
\mathrm{I}\end{array}$ & $\begin{array}{l}2 \\
2\end{array}$ & $\begin{array}{l}258.3 \\
128.0\end{array}$ & $\begin{array}{l}0.5 \\
0.2\end{array}$ & $\begin{array}{l}0.2142 \\
0.3742\end{array}$ & $\begin{array}{l}0.3 \\
0.2\end{array}$ & $\begin{array}{l}2.3 \\
0.5\end{array}$ & $\begin{array}{r}11.4 \\
1.5\end{array}$ & $\begin{array}{r}-0.019 \\
0.011\end{array}$ & (Hartkopf \& Mason 2010) \\
\hline 637 & AG 338 & HIP 43751 & 12.9210 & I & 2 & 167.9 & 0.1 & 2.1166 & 0.7 & 1.3 & & & h \\
\hline $08547+1637$ & TOK $196 \mathrm{Aa}, \mathrm{Ab}$ & HIP 43751 & 12.9210 & I & 2 & 149.6 & 2.0 & 0.1681 & 8.2 & 2.3 & & & \\
\hline \multirow{2}{*}{$08563-3707$} & RST2593 & HIP 43880 & 12.9209 & y & ${ }_{2}^{2}$ & 333.8 & 0.8 & 0.9276 & 0.5 & $2.8 *$ & -1.0 & -0.001 & (Hartkopf \& Mason 2011) \\
\hline & & & 12.9209 & I & 2 & 333.8 & 0.4 & 0.9278 & 0.5 & $3.7 *$ & -1.0 & -0.001 & 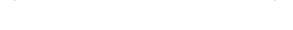 \\
\hline & HDS1297 & HIP 43983 & 13.1287 & I & 3 & 102.9 & 0.8 & 0.5711 & 0.8 & 2.5 & & & \\
\hline \multirow{3}{*}{$\begin{array}{l}08589+0829 \\
09024-6624\end{array}$} & $\begin{array}{l}\text { DEL } 2 \\
\text { TOK }\end{array}$ & GJ 3522 & $\begin{array}{l}12.9210 \\
13.1288\end{array}$ & I & 1 & 106.5 & 0.2 & 0.4612 & 0.2 & 0.7 & 1.5 & 0.164 & (Hartkopf et al. 2012) \\
\hline & TOK 197 & HIP 44382 & $\begin{array}{l}13.1288 \\
13.2380\end{array}$ & y & $\begin{array}{l}2 \\
3\end{array}$ & $\begin{array}{l}292.5 \\
280.8\end{array}$ & $\begin{array}{l}1.0 \\
0.5\end{array}$ & $\begin{array}{l}0.0284 \\
0.0290\end{array}$ & $\begin{array}{l}0.1 \\
0.3\end{array}$ & $\begin{array}{l}0.0 \\
0.1\end{array}$ & & & \\
\hline & HDS1326 & HIP 44745 & 13.1288 & y & 1 & 200.7 & $\begin{array}{l}.0 \\
1.4\end{array}$ & 0.7636 & $\begin{array}{l}.3 \\
1.4\end{array}$ & $1.9 *$ & & & \\
\hline \multirow{2}{*}{$09079-0708$} & & & 13.1288 & I & 2 & 200.8 & 0.5 & 0.7618 & 0.3 & $2.1 *$ & & & \\
\hline & TOK 64Aa,Ab & HIP 44804 & $\begin{array}{l}13.1286 \\
13.1286\end{array}$ & $\begin{array}{l}\mathrm{y} \\
\mathrm{I}\end{array}$ & $\begin{array}{l}2 \\
3\end{array}$ & $\begin{array}{l}307.7 \\
307.9\end{array}$ & $\begin{array}{l}3.3 \\
2.5\end{array}$ & $\begin{array}{l}0.4479 \\
0.4558\end{array}$ & $\begin{array}{l}3.3 \\
1.1\end{array}$ & $\begin{array}{l}4.1 \\
3.0\end{array}$ & & & \\
\hline
\end{tabular}


TABLE 2-Continued

\begin{tabular}{|c|c|c|c|c|c|c|c|c|c|c|c|c|c|}
\hline $\begin{array}{l}\text { WDS } \\
(2000)\end{array}$ & $\begin{array}{c}\text { Discoverer } \\
\text { Designation }\end{array}$ & $\begin{array}{l}\text { Other } \\
\text { name }\end{array}$ & $\begin{array}{l}\text { Epoch } \\
+2000 \\
\end{array}$ & Filt & $\mathrm{N}$ & $\begin{array}{c}\theta \\
(\mathrm{deg})\end{array}$ & $\begin{array}{l}\rho \sigma_{\theta} \\
\text { (mas) }\end{array}$ & $\begin{array}{l}\rho \\
\left({ }^{\prime \prime}\right)\end{array}$ & $\begin{array}{l}\sigma \rho \\
(\mathrm{mas})\end{array}$ & $\begin{array}{l}\Delta m \\
(\mathrm{mag})\end{array}$ & $\begin{array}{c}{[\mathrm{O}-\mathrm{C}]_{\theta}} \\
(\mathrm{deg})\end{array}$ & $\begin{array}{c}{\left[\begin{array}{l}\mathrm{O}-\mathrm{C}]_{\rho} \\
\left({ }^{\prime \prime}\right)\end{array}\right.} \\
\end{array}$ & $\begin{array}{c}\text { Reference } \\
\text { code* }^{*}\end{array}$ \\
\hline $09086-2550$ & RST2610AB & HIP 44874 & 13.1314 & I & 2 & 1.9 & 0.4 & 1.7946 & 6.5 & 4.8 & & & \\
\hline $09086-2550$ & TOK $357 \mathrm{BC}$ & HIP 44874 & $\begin{array}{l}13.1315 \\
13.1314\end{array}$ & ${ }_{\mathrm{I}}^{\mathrm{y}}$ & $\frac{1}{2}$ & $\begin{array}{r}1.9 \\
219.2\end{array}$ & $\begin{array}{l}0.0 \\
4.2\end{array}$ & $\begin{array}{l}1.7812 \\
0.1258\end{array}$ & $\begin{array}{l}0.0 \\
5.1\end{array}$ & $\begin{array}{l}7.2 \\
0.9\end{array}$ & & & \\
\hline & & & 13.1315 & & 1 & 231.5 & $\begin{array}{l}.2 \\
0.0\end{array}$ & 0.1185 & $\begin{array}{l}5.1 \\
0.0\end{array}$ & 7.5 & & & \\
\hline $09100-2845$ & В 179 & HIP 45003 & 13.1315 & I & 2 & 154.5 & 0.1 & 0.3416 & 0.2 & 0.3 & -4.5 & -0.053 & (Heintz 1993) \\
\hline $\begin{array}{l}09125-4032 \\
0925-4337\end{array}$ & $\begin{array}{l}\text { B } 1115 \\
\text { HJ } 4188 \mathrm{AB}\end{array}$ & $\begin{array}{l}\text { HIP } 45191 \\
\text { HIP } 45189\end{array}$ & $\begin{array}{l}13.1315 \\
13.1315\end{array}$ & $\mathrm{y}$ & $\begin{array}{l}2 \\
2\end{array}$ & $\begin{array}{l}135.1 \\
280.6\end{array}$ & $\begin{array}{l}0.1 \\
0.7\end{array}$ & $\begin{array}{l}0.1947 \\
2.8871\end{array}$ & $\begin{array}{l}0.7 \\
0.1\end{array}$ & $0.7 *$ & -2.2 & -0.010 & Table 7 \\
\hline $09128-6055$ & $\mathrm{HDO} 207 \mathrm{AB}$ & HIP 45214 & 13.1288 & y & 2 & 97.4 & 0.1 & 0.1390 & 0.3 & 0.4 & -2.3 & 0.004 & (Mason \& Hartkopf 2011a) \\
\hline $09173-6841$ & FIN $363 \mathrm{AB}$ & HIP 45571 & $\begin{array}{l}13.2380 \\
13.1288\end{array}$ & $\mathrm{y}$ & $\begin{array}{r}2 \\
2\end{array}$ & $\begin{array}{r}97.9 \\
114.9\end{array}$ & $\begin{array}{l}0.0 \\
0.4\end{array}$ & $\begin{array}{l}0.1388 \\
0.0529\end{array}$ & $\begin{array}{l}0.1 \\
0.1\end{array}$ & 0.4 & -2.1 & 0.004 & (Tokovinin 2012) \\
\hline $09191-4128$ & $\begin{array}{l}\text { CHR 239 } \\
\text { CHR }\end{array}$ & HIP 45705 & 13.1315 & y & 3 & $\begin{array}{l}288.2 \\
288.2\end{array}$ & 1.0 & 0.0701 & 1.1 & $\begin{array}{l}0.0 \\
2.3\end{array}$ & 0.1 & 0.000 & Table 6 inin 2012 \\
\hline & & & $\begin{array}{l}13.2379 \\
132270\end{array}$ & $\mathrm{y}$ & 2 & 278.7 & 0.5 & 0.0659 & 0.3 & 2.2 & -0.8 & -0.001 & \\
\hline $09193-5856$ & HDS1342 & HIP 45726 & 13.2379 & I & 2 & 279.1 & 0.4 & 0.0708 & 2.1 & 1.6 & -0.4 & 0.004 & \\
\hline & HDS1342 & HIP 45726 & 13.1288 & $\stackrel{\mathrm{y}}{\mathrm{I}}$ & 2 & 165.0 & 2.2 & 1.0265 & 2.2 & $3.6 *$ & & & \\
\hline $09194-7739$ & $\mathrm{KOH} 83 \mathrm{Aa}, \mathrm{Ab}$ & HIP 45734 & $\begin{array}{l}13.1288 \\
13.1288\end{array}$ & I & $\begin{array}{l}1 \\
2\end{array}$ & $\begin{array}{l}165.1 \\
153.4\end{array}$ & $\begin{array}{l}0.9 \\
1.1\end{array}$ & $\begin{array}{l}1.0247 \\
0.1225\end{array}$ & 0.9 & $4.6{ }^{*}$ & & & \\
\hline $09207-0742$ & A $1082 \mathrm{AB}$ & HD 80615 & 13.1286 & I & 2 & 207.3 & 0.6 & 0.4840 & 0.2 & 0.8 & -0.1 & 0.012 & (Docobo \& Ling 2011) \\
\hline $09228-0950$ & A $1342 \mathrm{AB}$ & HIP 45999 & 13.1286 & $\mathrm{y}$ & 2 & 203.9 & 0.2 & 0.1644 & 0.3 & 1.1 & -3.1 & 0.008 & Mason \& Hartkopf 2011a) \\
\hline $09243-3926$ & FIN 348 & HIP 46114 & 13.0796 & $\mathrm{y}$ & 2 & 260.7 & 0.1 & 0.1533 & 0.7 & 0.7 & 0.1 & -0.001 & Table 7 \\
\hline & WSI 73 & HLP 46191 & 13.2379 & $\mathrm{y}$ & 2 & 259.8 & 0.0 & 0.1535 & 0.0 & 0.4 & -0.1 & 0.000 & \\
\hline $\begin{array}{l}09252-1258 \\
09264-4215\end{array}$ & $\begin{array}{l}\text { WSI } 73 \\
\text { B } 1122\end{array}$ & $\begin{array}{l}\text { HIP } 46191 \\
\text { HIP } 46290\end{array}$ & $\begin{array}{l}13.1286 \\
13.0796\end{array}$ & $\mathrm{I}$ & $\begin{array}{l}2 \\
2\end{array}$ & $\begin{array}{l}279.4 \\
233.9\end{array}$ & $\begin{array}{l}0.5 \\
0.3\end{array}$ & 0.1197 & 1.4 & 0.4 & -0.7 & -0.004 & Table 7 \\
\hline $00204=4210$ & B 1122 & П1Р 46290 & 13.2379 & $\mathrm{y}$ & ${ }_{2}^{2}$ & $\begin{array}{l}233.9 \\
233.8\end{array}$ & $\begin{array}{l}.3 \\
0.0\end{array}$ & $\begin{array}{l}0.1523 \\
0.1556\end{array}$ & $\begin{array}{l}0.6 \\
0.1\end{array}$ & $\begin{array}{l}0.7 \\
0.3\end{array}$ & $\begin{array}{l}-0.1 \\
-1.0\end{array}$ & $\begin{array}{l}-0.004 \\
-0.001\end{array}$ & Table 7 \\
\hline $09275-5806$ & CHR 240 & HIP 46388 & 13.1287 & $\mathrm{y}$ & 2 & 292.9 & 2.6 & 0.0351 & 0.3 & 0.2 & & & \\
\hline & & & 13.2380 & $\mathrm{y}$ & 2 & 285.6 & 0.1 & 0.0378 & 0.5 & 0.4 & & & \\
\hline $\begin{array}{l}09278-0604 \\
09293-4432\end{array}$ & $\begin{array}{l}\text { B } 2530 \\
\text { HDS } 1360 \mathrm{Aa}, \mathrm{Ab}\end{array}$ & $\begin{array}{l}\text { HIP } 46404 \\
\text { HIP } 46523\end{array}$ & $\begin{array}{l}13.1286 \\
13.1315\end{array}$ & $\begin{array}{l}\mathrm{y} \\
\mathrm{y}\end{array}$ & $\frac{2}{2}$ & $\begin{array}{l}333.6 \\
155.8\end{array}$ & $\begin{array}{l}0.1 \\
0.4\end{array}$ & $\begin{array}{l}0.2807 \\
0.4153\end{array}$ & $\begin{array}{l}0.2 \\
0.9\end{array}$ & $\begin{array}{l}1.6 \\
3.0\end{array}$ & -14.0 & 0.160 & (Söderhjelm 1999) \\
\hline $09307-4028$ & COP 1 . & HIP 46651 & 13.0796 & y & 3 & 111.7 & 0.7 & 0.9680 & 1.7 & $0.9 *$ & -2.2 & -0.085 & (Mason \& Hartkopf 2001) \\
\hline $09313-1329$ & KUI 41 & HIP 46706 & 13.1286 & I & 2 & 279.4 & 0.4 & 0.7127 & 0.1 & 0.3 & 1.1 & 0.024 & (Söderhielm 1999) \\
\hline $09327+0152$ & FIN 349 & HIP 46840 & 13.1286 & $\mathrm{y}$ & 2 & $\begin{array}{r}215.8 \\
344.4\end{array}$ & 0.3 & 0.0533 & 0.3 & 0.3 & 4.2 & -0.002 & (Mason \& Hartkopf 2011a) \\
\hline $09383+0150$ & TOK 358 & HIP 47292 & $\begin{array}{l}13.1286 \\
13.1286\end{array}$ & $\stackrel{\text { y }}{\mathrm{I}}$ & $\frac{2}{2}$ & $\begin{array}{l}344.4 \\
344.3\end{array}$ & $\begin{array}{l}0.9 \\
1.5\end{array}$ & $\begin{array}{l}0.4533 \\
0.4525\end{array}$ & $\begin{array}{l}0.9 \\
0.7\end{array}$ & $\begin{array}{l}3.7 \\
3.0\end{array}$ & & & \\
\hline $09387-3937$ & I 202 & HIP 47328 & 13.0796 & $\mathrm{y}$ & 2 & 320.3 & 0.7 & 0.3183 & 1.0 & 2.6 & 1.3 & 0.018 & (Hartkopf et al. 2012) \\
\hline $09398-1034$ & CHR 175 & HIP 47427 & 13.1286 & $\mathrm{y}$ & 2 & & 0.2 & 0.3272 & 0.7 & 2.4 & & & \\
\hline $\begin{array}{l}09407-5759 \\
09415-1829\end{array}$ & $\begin{array}{l}\text { В } 780 \\
\text { TOK } 43 \mathrm{Aa}, \mathrm{Ab}\end{array}$ & $\begin{array}{l}\text { HIP } 47479 \\
\text { HIP } 47537\end{array}$ & $\begin{array}{l}13.1288 \\
13.1315\end{array}$ & $\mathrm{y}_{\mathrm{I}}$ & $\begin{array}{l}1 \\
1\end{array}$ & $\begin{array}{r}224.5 \\
25.3\end{array}$ & $\begin{array}{l}0.0 \\
0.1\end{array}$ & $\begin{array}{l}0.1148 \\
0.4242\end{array}$ & $\begin{array}{l}0.0 \\
0.1\end{array}$ & 0.4 & -4.7 & -0.004 & (DeRosa et al. 2012) \\
\hline $09415-1829$ & TOK $43 \mathrm{Aa}, \mathrm{Ab}$ & HIP 47537 & $\begin{array}{l}13.1315 \\
13.1315\end{array}$ & I & 1 & $\begin{array}{l}25.3 \\
25.5\end{array}$ & $\begin{array}{l}0.1 \\
0.0\end{array}$ & $\begin{array}{l}0.4242 \\
0.4303\end{array}$ & $\begin{array}{l}0.1 \\
0.0\end{array}$ & $\begin{array}{l}1.6 \\
1.7\end{array}$ & & & \\
\hline $09416-3830$ & TOK 198 & HIP 47543 & 13.1315 & I & 2 & & 1.6 & 0.4709 & 1.6 & 4.6 & & & \\
\hline $09442-2746$ & $\begin{array}{l}\text { FIN } 326 \\
\text { B } 1663\end{array}$ & $\begin{array}{l}\text { HIP } 47758 \\
\text { HIP } 48133\end{array}$ & 13.0795 & $\mathrm{y}$ & 1 & 14.1 & 0.1 & 0.1433 & 0.1 & $1.1 *$ & -0.4 & 0.000 & (Docobo \& Andrade 2013) \\
\hline $\begin{array}{l}09488-5237 \\
09495-1033\end{array}$ & $\begin{array}{ll}\text { B } 1663 \\
\text { A } 1344\end{array}$ & BD-08 2935 & $\begin{array}{l}13.1287 \\
13.1286\end{array}$ & $\begin{array}{l}1 \\
\mathrm{I}\end{array}$ & $\begin{array}{l}2 \\
2\end{array}$ & $\begin{array}{l}332.2 \\
279.4\end{array}$ & $\begin{array}{l}0.5 \\
0.2\end{array}$ & 0.2205 & 0.5 & 0.3 & 71.1 & 0.036 & (Heintz 1986b) \\
\hline $09505+0421$ & $\mathrm{RAO} 90 \mathrm{Ba}, \mathrm{Bb}$ & HIP $48273 \mathrm{~B}$ & 13.1286 & I & 4 & 83.2 & 0.8 & 0.1548 & 2.3 & 0.5 & H.1 & 0.050 & 10 \\
\hline $09527-7933$ & KOH 86 & HD 86588 & 13.1288 & I & 2 & 285.9 & 1.4 & 0.2927 & 1.3 & 1.9 & & & \\
\hline $09535+1657$ & CHR 219 & HIP 48504 & 13.1316 & I & 2 & 72.4 & 0.2 & 0.2534 & 0.7 & 0.5 & 0.3 & 0.002 & (Hartkopf et al. 2012) \\
\hline $10095-3551$ & RST 2678 & HIP 49769 & 13.1315 & I & 2 & 345.1 & 0.2 & 0.7755 & 0.2 & $3.7 *$ & & & \\
\hline $10120-2836$ & B 194 & HIP 49967 & 13.12 & $\mathrm{y}$ & 2 & 198.5 & 0.1 & 0.1 & 0.1 & 0.5 & -26.0 & 0.069 & (Seymour et al. 2002) \\
\hline $10161-2837$ & TOK 199 & HIP 50288 & 13.1289 & $\mathrm{y}$ & 2 & & 0.3 & 0.0511 & 0.3 & 0. & & & \\
\hline $\begin{array}{l}10161-5954 \\
10163-2859\end{array}$ & $\begin{array}{l}\text { HU } 1597 \\
\text { I } 851\end{array}$ & $\begin{array}{l}\text { HIP } 50287 \\
\text { HIP } 50309\end{array}$ & $\begin{array}{l}13.1315 \\
13.1289\end{array}$ & $\mathrm{y}$ & 2 & $\begin{array}{l}158.1 \\
235.6\end{array}$ & $\begin{array}{l}0.1 \\
0.0\end{array}$ & $\begin{array}{l}0.2884 \\
0.3836\end{array}$ & 0.1 & 0. & $\begin{array}{l}0.1 \\
0.8\end{array}$ & $\begin{array}{l}0.006 \\
0.002\end{array}$ & (Mason et al. 2009) \\
\hline $\begin{array}{l}10163-2859 \\
10183-0326\end{array}$ & $\begin{array}{l}1851 \\
\text { RST } 4454 \mathrm{AB}\end{array}$ & HD 89334 & $\begin{array}{l}13.1289 \\
13.1316\end{array}$ & $\begin{array}{l}\mathrm{y} \\
\mathrm{I}\end{array}$ & $\frac{2}{2}$ & $\begin{array}{l}235.6 \\
223.3\end{array}$ & $\begin{array}{l}0.0 \\
0.2\end{array}$ & $\begin{array}{l}0.3836 \\
0.2919\end{array}$ & $\begin{array}{l}0.2 \\
0.2\end{array}$ & $\begin{array}{l}0.3 \\
0.5\end{array}$ & $\begin{array}{l}0.8 \\
0.2\end{array}$ & $\begin{array}{l}0.002 \\
0.002\end{array}$ & (Hartkopf \& Mason $\frac{2010)}{2011)}$ \\
\hline 102 & BU & HIP $50^{\circ}$ & 13.1 & I & 3 & 13 & 0.3 & 1.5 & 0.8 & $0.6 *$ & & & \\
\hline $10282-2548$ & FIN $308 \mathrm{AB}$ & HIP 51255 & 13.1289 & $\mathrm{y}$ & 2 & & 3.8 & 0.1300 & 1.5 & 0.6 & 178.4 & 0.006 & (Hartkopf et al. 2012) \\
\hline $\begin{array}{l}10282-2548 \\
10311-2411\end{array}$ & $\begin{array}{l}\text { B } 199 \mathrm{AC} \\
\text { B } 201 \mathrm{AB}\end{array}$ & $\begin{array}{l}\text { HIP } 51255 \\
\text { HIP } 51501\end{array}$ & $\begin{array}{l}13.1289 \\
13.1289\end{array}$ & $\begin{array}{l}\mathrm{y} \\
\mathrm{y}\end{array}$ & $\begin{array}{l}2 \\
1\end{array}$ & $\begin{array}{r}174.4 \\
68.0\end{array}$ & $\begin{array}{l}0.5 \\
0.4\end{array}$ & $\begin{array}{l}1.1526 \\
1.9822\end{array}$ & $\begin{array}{l}3.0 \\
0.4\end{array}$ & $3.7 \%$ & & & \\
\hline & & & 13.1289 & I & 1 & $\begin{array}{l}68.0 \\
68.0\end{array}$ & 0.2 & $\begin{array}{l}1.9822 \\
1.9815\end{array}$ & $\begin{array}{l}0.4 \\
0.2\end{array}$ & $4.2 *$ & & & \\
\hline $10361-$ & BU 41 & HIP 51885 & $13.12>>2$ & $\mathrm{y}$ & 2 & 305.7 & 0. & & 0.3 & $1.2 *$ & 0.1 & -0.004 & Table 7 \\
\hline $10370-$ & TOK & 19 & 13.1316 & y & 1 & 81 & 0.0 & 0.1245 & 0. & 2 & & & \\
\hline & & & & I & 2 & & 4.3 & & & & & & \\
\hline $10370-0850$ & A $556 \mathrm{Aa}, \mathrm{B}$ & HIP 51966 & 13.1 & $\mathrm{y}$ & 1 & 19 & 0.0 & 0.9080 & 0.0 & 2. & -0.8 & 0.015 & Table 7 \\
\hline & & & 13.1316 & I & 2 & 198.6 & 3.8 & 0.9056 & 4.0 & 2.2 & -1.0 & 0.013 & \\
\hline $10375-0932$ & RST3708 & HD 92015 & 13.1316 & I & 2 & 355.5 & 0.3 & 0.5007 & 0.2 & $1.3 * * 2 * 0$ & -74.1 & 0.258 & (Heintz 1991) \\
\hline $10415-2705$ & В 203 & HIP 52325 & $\begin{array}{l}13.1289 \\
13.1289\end{array}$ & $\stackrel{\mathrm{y}}{\mathrm{y}}$ & ${ }_{1}^{2}$ & $\begin{array}{l}15.1 \\
15.1\end{array}$ & $\begin{array}{l}0.3 \\
0.2\end{array}$ & $\begin{array}{l}0.9836 \\
0.9839\end{array}$ & $\begin{array}{l}0.3 \\
0.2\end{array}$ & $\begin{array}{l}2.2 * \\
2.3 *\end{array}$ & & & \\
\hline $10419-7811$ & HDS1530 & HIP 52351 & 13.1288 & I & 2 & $\begin{array}{l}105.4 \\
165.4\end{array}$ & 0.4 & 0.1685 & 0.6 & 0.7 & & & \\
\hline
\end{tabular}


TABLE 2-Continued

\begin{tabular}{|c|c|c|c|c|c|c|c|c|c|c|c|c|c|}
\hline $\begin{array}{l}\text { WDS } \\
(2000)\end{array}$ & $\begin{array}{c}\text { Discoverer } \\
\text { Designation }\end{array}$ & $\begin{array}{l}\text { Other } \\
\text { name }\end{array}$ & $\begin{array}{l}\text { Epoch } \\
+2000\end{array}$ & Filt & $\mathrm{N}$ & $\begin{array}{c}\theta \\
(\mathrm{deg})\end{array}$ & $\begin{array}{l}\rho \sigma_{\theta} \\
(\mathrm{mas})\end{array}$ & $\begin{array}{l}\rho \\
\left({ }^{\prime \prime}\right)\end{array}$ & $\begin{array}{c}\sigma \rho \\
\text { (mas) }\end{array}$ & $\begin{array}{l}\Delta m \\
(\mathrm{mag})\end{array}$ & 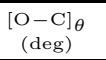 & $\begin{array}{c}{[\mathrm{O}-\mathrm{C}]_{\rho}} \\
\left({ }^{\prime \prime}\right)\end{array}$ & $\begin{array}{c}\text { Reference } \\
\text { code* }\end{array}$ \\
\hline $10426+0335$ & A 2768 & HIP 52401 & 13.1316 & & 2 & 246.6 & 0.2 & 0.6137 & 0.1 & 1.7 & 0.4 & 0.010 & (Hartkopf \& Mason 2010) \\
\hline \multirow{4}{*}{$\begin{array}{l}10446+0530 \\
10465-6416\end{array}$} & A 2771 & HIP 52547 & 13.1316 & I & 2 & 117.6 & 0.2 & 0.4661 & 0.2 & 0.7 & -0.5 & 0.015 & Table 7 \\
\hline & FIN $364 \mathrm{AB}$ & HIP 52701 & 13.1288 & $\mathrm{y}$ & 2 & 139.5 & 6.0 & 0.0840 & 2.8 & 0.8 & 3.1 & 0.011 & (Hartkopf et al. 2012) \\
\hline & & & 13.1288 & I & 2 & 139.8 & 1.8 & 0.0757 & 1.4 & 0.8 & 3.4 & 0.002 & \\
\hline & & & $\begin{array}{l}13.1315 \\
13.1316\end{array}$ & $\begin{array}{l}\mathrm{I} \\
\mathrm{y}\end{array}$ & $\begin{array}{l}2 \\
2\end{array}$ & $\begin{array}{l}139.1 \\
137.2\end{array}-10$ & $\begin{array}{l}3.5 \\
0.6\end{array}$ & $\begin{array}{l}0.0804 \\
0.0852\end{array}-1$ & $\begin{array}{l}2.6 \\
0.8\end{array}$ & $\begin{array}{l}0.9 \\
1.0\end{array}$ & $\begin{array}{l}2.7 \\
0.7\end{array}$ & $\begin{array}{l}0.007 \\
0.012\end{array}$ & \\
\hline \multirow{3}{*}{$10465-6416$} & TOK $45 \mathrm{AC}$ & HIP 52701 & $\begin{array}{l}13.1316 \\
13.1288\end{array}$ & $\begin{array}{l}\mathrm{y} \\
\mathrm{y}\end{array}$ & $\begin{array}{l}2 \\
2\end{array}$ & $\begin{array}{r}137.2 \\
14.6\end{array}$ & $\begin{array}{l}0.6 \\
0.1\end{array}$ & $\begin{array}{l}0.0852 \\
0.7014\end{array}$ & $\begin{array}{l}5.8 \\
5.7\end{array}$ & $\begin{array}{l}1.0 \\
4.2\end{array}$ & & & \\
\hline & & & 13.1288 & I & 2 & 14.9 & 0.1 & 0.7025 & 1.3 & 3.6 & & & \\
\hline & & & $\begin{array}{l}13.1315 \\
13.1316\end{array}$ & $\stackrel{\mathrm{I}}{\mathrm{y}}$ & $\begin{array}{l}2 \\
2\end{array}$ & $\begin{array}{l}14.2 \\
14.4\end{array}$ & $\begin{array}{l}0.4 \\
1.1\end{array}$ & $\begin{array}{l}0.7068 \\
0.7061\end{array}$ & $\begin{array}{l}1.3 \\
2.8\end{array}$ & $\begin{array}{l}3.7 \\
4.5\end{array}$ & & & \\
\hline $10468-4925$ & R 155 & HIP 52727 & $\begin{array}{l}13.1316 \\
13.1315\end{array}$ & $\underset{\mathrm{I}}{\mathrm{y}}$ & $\frac{2}{2}$ & $\begin{array}{l}14.4 \\
5.9\end{array}-\longrightarrow>10$ & $\begin{array}{l}1.1 \\
1.0\end{array}$ & & $\begin{array}{l}2.8 \\
1.2\end{array}$ & $\begin{array}{l}4.5 * 2 * \\
4.2 * 10\end{array}$ & 0.1 & -0.074 & Table 7 \\
\hline $10529-1717$ & HDS1556 & HIP 53206 & 13.1289 & $\mathrm{y}$ & 2 & 255.8 & 0.4 & 0.0579 & 0.4 & 0.1 & 12.3 & 0.006 & (Hartkopf et al. 2012) \\
\hline & & HIP 53423 & 13.1316 & $\mathrm{y}$ & 2 & 52.5 & 0.3 & 1.1454 & 0.5 & $3.2 *$ & 0.1 & 0.019 & (Hartkopf \& Mason 2010) \\
\hline $10592-8133$ & I 212 & HIP 53700 & 13.1288 & $\mathrm{y}$ & 2 & 5.8 & 0.1 & 0.3406 & 0.2 & 0.5 & 0.2 & 0.004 & Table 7 \\
\hline $11009-4030$ & FIN 365 & HIP 53840 & 13.2380 & $\mathrm{y}$ & 2 & 109.0 & 0.1 & 0.1589 & 0.1 & 0.4 & 1.7 & 0.007 & (Tokovinin 2012) \\
\hline $11014-1204$ & HDS1572 & HIP 53879 & 13.1290 & $\mathrm{y}$ & 2 & 120.1 & 1.8 & 0.0301 & 1.2 & 0.9 & & & \\
\hline $11042-5828$ & JSP $459 \mathrm{AB}$ & HD 96118 & 13.1288 & I & 2 & 122.6 & 1.9 & 2.2892 & 1.9 & $5.4 *$ & & & \\
\hline $\begin{array}{l}11056-1105 \\
11101-3822\end{array}$ & $\begin{array}{l}\text { TOK } 221 \\
\text { B } 795\end{array}$ & $\begin{array}{l}\text { HIP } 54214 \\
\text { HIP } 54577\end{array}$ & 13.2381 & I & 2 & $\begin{array}{r}60.2 \\
1870\end{array}$ & 2.1 & 0.5967 & 5.5 & $5.4 *$ & & & \\
\hline $\begin{array}{l}11101-3822 \\
1102-1122\end{array}$ & $\begin{array}{l}\text { B } 795 \\
\text { HDS1590 }\end{array}$ & $\begin{array}{l}\text { HIP } 54577 \\
\text { HIP } 54580\end{array}$ & $\begin{array}{l}13.2380 \\
13.1290\end{array}$ & $\begin{array}{l}\mathrm{y} \\
\mathrm{y}\end{array}$ & $\begin{array}{l}2 \\
2\end{array}$ & $\begin{array}{l}187.0 \\
312.1\end{array}-\quad-50$ & $\begin{array}{l}0.2 \\
0.1\end{array}$ & $\begin{array}{l}0.7473 \\
0.1037\end{array}$ & $\begin{array}{l}0.3 \\
1.1\end{array}$ & $\begin{array}{l}1.9 x^{*}-4 x-3 \\
0.4\end{array}$ & 2.0 & 0.010 & \\
\hline \multirow{2}{*}{$11125-1830$} & $\begin{array}{l}\text { HDST590 } \\
\text { BU } 220\end{array}$ & HIP 54742 & 13.1289 & $\begin{array}{l}\mathrm{y} \\
\mathrm{y}\end{array}$ & 2 & 316.0 & 0.0 & 0.2720 & $\begin{array}{l}1.1 \\
0.2\end{array}$ & 1.2 & 16.8 & 0.182 & (Heintz 1995) \\
\hline & & & 13.2380 & I & 2 & 315.9 & 0.0 & 0.2715 & 0.0 & 0.8 & 17.4 & 0.184 & \\
\hline $11170-0708$ & $\mathrm{BU} 600 \mathrm{AB}$ & HIP 55106 & 13.2381 & I & 2 & 210.7 & 0.7 & 1.3302 & 0.7 & $3.9 *$ & & & \\
\hline $11175-5906$ & RST $4472 \mathrm{BC}$ & HIP 55149 & 13.1288 & I & 3 & 33.3 & 2.9 & 0.2870 & 4.4 & 1.0 & & & \\
\hline $11175-5906$ & R $163 \mathrm{AB}$ & HIP 55149 & 13.1288 & I & 3 & 58.1 & 4.5 & 1.6098 & 2.8 & 0.9 & & & \\
\hline \multirow[t]{2}{*}{$11192-1950$} & TOK $383 \mathrm{Aa}, \mathrm{Ab}$ & HD 98412 & $\begin{array}{l}13.2380 \\
132381\end{array}$ & I & $\begin{array}{r}3 \\
2\end{array}$ & 26.0 & 0.4 & 0.0564 & 2.9 & 0.1 & & & \\
\hline & & & $\begin{array}{l}13.2381 \\
13.2381\end{array}$ & $\mathrm{y}$ & $\begin{array}{l}2 \\
2\end{array}$ & $\begin{array}{l}25.8 \\
25.9\end{array}$ & $\begin{array}{l}0.2 \\
0.6\end{array}$ & $\begin{array}{l}0.0518 \\
0.0480\end{array}-10$ & $\begin{array}{l}0.2 \\
0.2\end{array}$ & $\begin{array}{l}0.3 \\
0.0:\end{array}$ & & & \\
\hline $11210-5429$ & I 879 & HIP 55425 & 13.1288 & $\mathrm{y}$ & 2 & 86.3 & 0.1 & 0.1571 & 0.0 & 1.5 & -0.4 & 0.000 & (Tokovinin 2012) \\
\hline $11221-2447$ & I $507 \mathrm{AB}$ & HIP 55505 & 13.1289 & I & 2 & 5.3 & 0.1 & 0.6300 & 0.2 & 0.3 & 0.4 & -0.167 & (Tokovinin 1999) \\
\hline $11250-3200$ & & & $\begin{array}{l}13.1289 \\
13.2380\end{array}$ & $\mathrm{R}$ & $\frac{1}{2}$ & $\begin{array}{r}5.3 \\
1316\end{array}$ & 0.1 & 0.6291 & 0.1 & 0.6 & 0.4 & -0.168 & \\
\hline $\begin{array}{l}11250-3200 \\
11268-5310\end{array}$ & $\begin{array}{l}\text { CHR } 242 \mathrm{Aa}, \mathrm{Ab} \\
\text { I } 883\end{array}$ & $\begin{array}{l}\text { HIP } 55714 \\
\text { HIP } 55849\end{array}$ & $\begin{array}{l}13.2380 \\
13.1288\end{array}$ & $\mathrm{y}$ & $\begin{array}{r}2 \\
2\end{array}$ & $\begin{array}{l}131.6 \\
232.1\end{array}$ & $\begin{array}{l}0.1 \\
0.1\end{array}$ & $\begin{array}{l}0.1418 \\
0.2354\end{array}$ & $\begin{array}{l}0.2 \\
0.2\end{array}$ & $\begin{array}{l}1.4 \\
0.6\end{array}$ & 0.3 & -0.003 & (Hartkopf \& Mason 2010) \\
\hline $11272-1539$ & $\begin{array}{l}1883 \\
\text { HU } 462\end{array}$ & HIP 55875 & 13.1289 & $\begin{array}{l}\mathrm{y} \\
\mathrm{y}\end{array}$ & $\frac{2}{2}$ & 97.0 & 0.2 & 0.4274 & 0.1 & 0.6 & -2.9 & 0.027 & (Mason et al. 2006) \\
\hline \multirow{4}{*}{$\begin{array}{l}11318-0142 \\
11318-2047\end{array}$} & RST4944 & HIP 55941 & 13.2381 & $\mathrm{y}$ & 2 & 227.8 & 0.3 & 0.1339 & 0.8 & 1.9 & -0.8 & -0.001 & Hartkopf \& Mason \\
\hline & TOK 222 & HIP 56245 & 13.2381 & I & 2 & $\begin{array}{r}227.8 \\
65.9\end{array}$ & $\begin{array}{l}1.3 \\
1.2\end{array}$ & 1.0683 & $\begin{array}{l}.8 \\
1.2\end{array}$ & 4.8 * 1.90 & -0.0 & -0.001 & HartKopr \& Mrason 2U10 \\
\hline & & & 13.1289 & & 1 & 65.9 & 2.9 & 1.0633 & 2.9 & $5.1 *$ & & & \\
\hline & & & 13.1289 & I & 2 & 65.8 & 1.9 & 1.0683 & 1.9 & $5.5 *$ & & & \\
\hline \multirow{4}{*}{$11342+1101$} & I 1544 & HIP 56326 & 13.2380 & I & 2 & 63.5 & 1 & & 04 & $4.3 *$ & & & \\
\hline & HDS1642AB & HIP 56429 & 13.1290 & & 2 & 246.1 & 0.3 & 1.1310 & 0.6 & $2.7 *$ & & & \\
\hline & & & 13.2381 & I & 2 & 246.1 & 0.3 & 1.1312 & 1.6 & $2.2 *$ & & & \\
\hline & & & 13.2381 & & 2 & 246.3 & 0.6 & 1.1311 & 0.6 & $3.0 *$ & & & \\
\hline $11387-1312$ & KUI $58 \mathrm{Aa}, \mathrm{Ab}$ & HIP 56802 & 13.1290 & I & 3 & 248.7 & 0.4 & 1.0397 & 0.4 & $4.3 *$ & & & \\
\hline \multirow{3}{*}{$11420-1701$} & B $1705 \mathrm{AB}$ & HIP 56862 & 13.1288 & $\mathrm{y}$ & 2 & 92.5 & 0.1 & 0.2000 & 0.4 & 1.5 & 0.5 & -0.001 & Table 7 \\
\hline & TOK $384 \mathrm{Aa}, \mathrm{Ab}$ & HIP 57078 & 13.2381 & $\mathrm{y}$ & 2 & 128.5 & 1.2 & 0.1467 & 0.8 & 3.6 & & & \\
\hline & & & 13.2381 & I & 2 & 131.0 & 2.3 & 0.1516 & 2.4 & 2.9 & & & \\
\hline $\begin{array}{l}11420-1701 \\
11425+2355\end{array}$ & TOK $384 \mathrm{Aa}, \mathrm{B}$ & HIP 57078 & 13.2381 & I & 2 & 95.0 & 0.1 & 1.1089 & 3.2 & 5.3 & & & \\
\hline & & & & $\begin{array}{l}1 \\
\text { I }\end{array}$ & $\begin{array}{r}2 \\
2\end{array}$ & $\begin{array}{l}261.9 \\
199.5\end{array}$ & 02 & $\begin{array}{l}0.2995 \\
0.2085\end{array}$ & 0. & 1.6 & & & \\
\hline $\begin{array}{l}11446-4925 \\
11495-4604\end{array}$ & $\begin{array}{l}\text { RST9004AB } \\
\text { FIN 366 }\end{array}$ & $\begin{array}{l}\text { HIP } 57269 \\
\text { HIP } 57657\end{array}$ & $\begin{array}{l}13.1288 \\
13.1288\end{array}$ & $\mathrm{y}$ & 2 & $\begin{array}{r}199.5 \\
80.4\end{array}$ & $\begin{array}{l}0.2 \\
0.1\end{array}$ & 0.2085 & $\begin{array}{l}0.4 \\
0.3\end{array}$ & 1.2 & -51.1 & 0.017 & (Heintz 1986b) \\
\hline \multirow{3}{*}{$\begin{array}{l}11514+1148 \\
11525-1408\end{array}$} & $\begin{array}{l}\text { FIN } 366 \\
\text { HDS1672 }\end{array}$ & $\begin{array}{l}\text { HIP } 57657 \\
\text { HIP } 57821\end{array}$ & $\begin{array}{l}13.1288 \\
13.1290\end{array}$ & $\mathrm{y}$ & 2 & $\begin{array}{l}80.4 \\
96.0\end{array}$ & $\begin{array}{l}0.1 \\
0.8\end{array}$ & $\begin{array}{l}0.0844 \\
0.2146\end{array}$ & 0. & $\begin{array}{l}1.3 \\
3.7\end{array}$ & $\begin{array}{l}-1.8 \\
-0.8\end{array}$ & $\begin{array}{r}-0.004 \\
0.002\end{array}$ & $\begin{array}{l}\text { Table } 7 \\
\text { Table } 6\end{array}$ \\
\hline & & & 13.1290 & I & 2 & 95.4 & $\begin{array}{l}0.8 \\
1.1\end{array}$ & 0.2147 & 0. & $\begin{array}{l}3.1 \\
3.3\end{array}$ & $\begin{array}{l}-0.8 \\
-1.4\end{array}$ & 0.002 & \\
\hline & HDS1676 & HIP 57894 & 13.1290 & $\mathrm{y}$ & 2 & 133.5 & 0.3 & 0.1697 & 0.6 & 1.4 & -0.1 & 0.000 & Table 6 \\
\hline & & & 381 & $\mathrm{y}$ & 2 & 134.3 & 0.1 & 0.1676 & 0.1 & 1.3 & -0.2 & 0.001 & \\
\hline & $\mathrm{HV}$ & $\mathrm{H}$ & & $\mathrm{y}$ & 2 & 89.5 & 0. & 0.2772 & 0.2 & 1.9 & & & \\
\hline 439 & I 215 & HIP 58 & 13.1316 & $\mathrm{y}$ & 2 & 41.4 & 0.0 & 0.2407 & 0.0 & 1.1 & -0.7 & -0.012 & Table 7 \\
\hline-3901 & SEE 143 & HIP 58799 & 13.1316 & $\mathrm{y}$ & 2 & 28.3 & 0.0 & 0.5200 & 0.2 & 0.6 & -0.3 & 0.007 & Table 7 \\
\hline $\begin{array}{l}12270-0332 \\
12283-6146\end{array}$ & $\begin{array}{l}\text { A } 79 \mathrm{AB} \\
\mathrm{CPO} 12 \mathrm{AB}\end{array}$ & $\begin{array}{l}\text { HIP } 60749 \\
\text { HIP } 60845\end{array}$ & $\begin{array}{l}13.1290 \\
131318\end{array}$ & $\mathrm{y}$ & & $\begin{array}{r}98.8 \\
188.4\end{array}$ & $\begin{array}{l}0.2 \\
0.0\end{array}$ & $\begin{array}{l}0.4816 \\
1.9783\end{array}$ & $\begin{array}{l}0.2 \\
0.0\end{array}$ & 1.1 & $\begin{array}{l}10.1 \\
1.1\end{array}$ & 0.112 & (Mante 2003) \\
\hline $\begin{array}{l}12283-6146 \\
12283-6146\end{array}$ & RST $4499 \mathrm{BC}$ & $\begin{array}{l}\text { HIP } 60845 \\
\text { HIP } 60845\end{array}$ & $\begin{array}{l}13.1318 \\
13.1318\end{array}$ & $\begin{array}{l}\mathrm{y} \\
\mathrm{y}\end{array}$ & $\begin{array}{l}1 \\
1\end{array}$ & $\begin{array}{l}188.4 \\
170.9\end{array}$ & $\begin{array}{l}0.0 \\
0.0\end{array}$ & $\begin{array}{l}1.9783 \\
0.2145\end{array}$ & $\begin{array}{l}0.0 \\
0.0\end{array}$ & $\begin{array}{l}2.6 \\
0.4\end{array}$ & $\begin{array}{l}1.1 \\
1.1\end{array}$ & $\begin{array}{l}-0.127 \\
-0.003\end{array}$ & $\begin{array}{l}\text { (Seymour et al. } 2002 \text { ) } \\
\text { Table } 7\end{array}$ \\
\hline & & & & $\mathrm{y}$ & 1 & 170.7 & 0. & 0.2125 & 0.1 & $\begin{array}{l}0.4 \\
0.6\end{array}$ & 0.9 & 55 & \\
\hline $12314-5659$ & RST2802 & HD 108938 & 13.2382 & I & 2 & 50.8 & 0.3 & 1.2342 & 1.1 & $1.9 *$ & & & \\
\hline $12392-4022$ & $\begin{array}{l}\text { B } 1215 \\
1215\end{array}$ & HIP 61729 & 13.1317 & $\mathrm{y}$ & 2 & 357.7 & 0.1 & 0.2383 & 0.1 & 0.4 & -1.2 & 0.010 & Rica Romero 2010) \\
\hline
\end{tabular}


TABLE 2-Continued

\begin{tabular}{|c|c|c|c|c|c|c|c|c|c|c|c|c|c|}
\hline $\begin{array}{l}\text { WDS } \\
(2000)\end{array}$ & $\begin{array}{c}\text { Discoverer } \\
\text { Designation }\end{array}$ & $\begin{array}{l}\text { Other } \\
\text { name }\end{array}$ & $\begin{array}{c}\text { Epoch } \\
+2000 \\
\end{array}$ & Filt & $\mathrm{N}$ & $\begin{array}{c}\theta \\
(\mathrm{deg})\end{array}$ & $\begin{array}{l}\rho \sigma_{\theta} \\
(\mathrm{mas})\end{array}$ & $\begin{array}{l}\rho \\
\left(^{\prime \prime}\right)\end{array}$ & $\begin{array}{c}\sigma \rho \\
\text { (mas) }\end{array}$ & $\begin{array}{l}\Delta m \\
(\mathrm{mag})\end{array}$ & $\begin{array}{c}{[\mathrm{O}-\mathrm{C}]_{\theta}} \\
(\mathrm{deg})\end{array}$ & $\begin{array}{c}{[\mathrm{O}-\mathrm{C}]_{\rho}} \\
\left({ }^{\prime \prime}\right)\end{array}$ & $\begin{array}{c}\text { Reference } \\
\text { code* }\end{array}$ \\
\hline $12415-4858$ & $\mathrm{HJ} 4539 \mathrm{AB}$ & HIP 61932 & 13.2382 & & 2 & 224.2 & 0.1 & 0.1442 & 0.1 & 0.2 & -36.0 & -0.003 & \\
\hline $12417-0127$ & STF1670AB & HIP 61941 & 13.1290 & $\mathrm{y}$ & 3 & 10.4 & 0.3 & 1.9672 & 0.3 & $0.1 *$ & -0.3 & -0.006 & Scardia et al. 2007) \\
\hline $12446-5717$ & FIN $65 \mathrm{AB}$ & HIP 62179 & 13.2382 & $\mathrm{y}$ & 2 & 86.6 & 0.1 & 0.2881 & 0.2 & 1.2 & 0.0 & -0.002 & Docobo \& Andrade 2013) \\
\hline $12452-4656$ & HDS1787 & HIP 62228 & 13.2382 & I & 2 & 295.3 & 0.7 & 0.0716 & 0.6 & $0.2 *$ & & & \\
\hline $\begin{array}{l}12463-6806 \\
12485-1543\end{array}$ & $\begin{array}{l}\text { R } 207 \\
\text { WSI } 74 \mathrm{Aa}, \mathrm{Ab}\end{array}$ & $\begin{array}{l}\text { HIP } 62322 \\
\text { HIP } 62505\end{array}$ & $\begin{array}{l}13.1318 \\
13.1290\end{array}$ & $\begin{array}{l}\mathrm{y} \\
\mathrm{y}\end{array}$ & ${ }_{2}^{2}$ & $\begin{array}{r}50.3 \\
150.4\end{array}$ & $\begin{array}{l}0.1 \\
0.2\end{array}$ & $\begin{array}{l}1.0408 \\
0.0991\end{array}$ & $\begin{array}{l}0.7 \\
0.2\end{array}$ & $\begin{array}{l}0.5 * \\
1.6\end{array}$ & $\begin{array}{l}-6.0 \\
-2.9\end{array}$ & $\begin{array}{r}0.124 \\
-0.003\end{array}$ & $($ Raca 2012$)$ \\
\hline $\begin{array}{l}12455-1543 \\
12509-5743\end{array}$ & $\begin{array}{l}\text { WSI 74Aa, Ab } \\
\text { TOK } 385\end{array}$ & $\begin{array}{l}\text { HIP } 62505 \\
\text { HIP } 62699\end{array}$ & $\begin{array}{l}13.1290 \\
13.2382\end{array}$ & $\stackrel{\mathrm{y}}{\mathrm{I}}$ & $\begin{array}{l}2 \\
2\end{array}$ & $\begin{array}{l}150.4 \\
112.9\end{array}$ & $\begin{array}{l}0.2 \\
2.2\end{array}$ & $\begin{array}{l}0.0991 \\
0.1868\end{array}$ & $\begin{array}{l}0.2 \\
4.9\end{array}$ & $\begin{array}{l}1.6 \\
3.6\end{array}$ & -2.9 & -0.003 & Table 6 \\
\hline $13081-6518$ & $\mathrm{CHR} 247 \mathrm{Aa}, \mathrm{Ab}$ & HIP 64094 & 13.1291 & $\mathrm{y}$ & 2 & 148.0 & 2.0 & 0.0461 & 1.1 & 0.0 & & & \\
\hline & & & $\begin{array}{l}13.2382 \\
13.1317\end{array}$ & $\begin{array}{l}\mathrm{y} \\
\mathrm{y}\end{array}$ & ${ }_{2}^{2}$ & $\begin{array}{l}106.7 \\
116.2\end{array}$ & $\begin{array}{l}2.1 \\
0.4\end{array}$ & $\begin{array}{l}0.0258 \\
0.1947\end{array}$ & $\begin{array}{l}2.7 \\
0.4\end{array}$ & 0.7 & & & \\
\hline $13106-3128$ & RST1706 & HIP 64292 & $\begin{array}{l}13.1317 \\
13.1317\end{array}$ & $\begin{array}{l}\mathrm{y} \\
\mathrm{I}\end{array}$ & $\begin{array}{l}2 \\
1\end{array}$ & $\begin{array}{l}116.2 \\
116.1\end{array}$ & $\begin{array}{l}0.4 \\
0.1\end{array}$ & $\begin{array}{l}0.1947 \\
0.1962\end{array}$ & $\begin{array}{l}0.4 \\
0.1\end{array}$ & $\begin{array}{l}2.2 \\
1.6\end{array}$ & & & \\
\hline $13123-5955$ & SEE $170 A B$ & HIP 64425 & 13.1291 & $\mathrm{y}$ & $\frac{1}{2}$ & $\begin{array}{l}119.1 \\
119.6\end{array}$ & $\begin{array}{l}0.1 \\
0.1\end{array}$ & 0.2185 & 0.5 & $\begin{array}{l}1.0 \\
1.8\end{array}$ & -3.9 & 0.009 & (Finsen 1964) \\
\hline $13126-6034$ & WSI $75 \mathrm{Aa}, \mathrm{Ab}$ & U1312-6033 & 13.1291 & $\mathrm{y}$ & 1 & 83.9 & 0.4 & 0.0837 & 0.4 & 2.9 & & & \\
\hline & & & $\begin{array}{l}13.1291 \\
13.2382\end{array}$ & R & $\frac{1}{2}$ & $\begin{array}{l}84.7 \\
84.0\end{array}$ & 0.6 & $\begin{array}{l}0.0783 \\
0.0845\end{array}$ & 0.6 & 3.2 & & & \\
\hline $13132-0233$ & TOK $69 \mathrm{Aa}, \mathrm{Ab}$ & HIP 64498 & $\begin{array}{l}13.2382 \\
13.1291\end{array}$ & $\begin{array}{l}1 \\
\mathrm{y}\end{array}$ & ${ }_{1}^{2}$ & $\begin{array}{r}84.0 \\
307.8\end{array}$ & $\begin{array}{l}1.7 \\
1.3\end{array}$ & $\begin{array}{l}0.385 \\
0.3810\end{array}$ & $\begin{array}{l}6.6 \\
1.3\end{array}$ & $\begin{array}{l}2.1 \\
4.4\end{array}$ & & & \\
\hline & & (1) & 13.1291 & I & 2 & 307.3 & 2.1 & 0.3829 & 0.7 & $\begin{array}{l}\text { a.4 } \\
3.1\end{array}$ & & & \\
\hline $13137-6248$ & HDS1852 & HIP 64537 & $\begin{array}{l}13.1291 \\
13.1291\end{array}$ & $\mathrm{y}$ & $\frac{1}{2}$ & $\begin{array}{l}136.7 \\
136.7\end{array}$ & 0.2 & $\begin{array}{l}0.1863 \\
0.1870\end{array}$ & $\begin{array}{l}0.2 \\
10\end{array}$ & $\begin{array}{l}0.7 \\
0.4\end{array}$ & & & \\
\hline $13138-6335$ & WSI 57 & HD 114737 & $\begin{array}{l}13.1291 \\
13.2383\end{array}$ & I & $\begin{array}{l}2 \\
2\end{array}$ & $\begin{array}{r}36.7 \\
54.0\end{array}$ & $\begin{array}{l}0.2 \\
3.0\end{array}$ & $\begin{array}{l}0.1870 \\
0.1932\end{array}$ & $\begin{array}{l}1.0 \\
1.2\end{array}$ & $\begin{array}{l}0.4 \\
2.4\end{array}$ & & & \\
\hline $13145-2417$ & FIN $297 \mathrm{AB}$ & HIP 64603 & 13.1317 & $\mathrm{y}$ & 2 & $\begin{array}{r}7.3 \\
2.8\end{array}$ & 0.0 & 0.1793 & 0.2 & 0.4 & 1.5 & -0.044 & (Mason et al. 2010) \\
\hline $13147-6335$ & WSI $58 \mathrm{Aa}, \mathrm{Ab}$ & HIP 64624 & $\begin{array}{l}13.1291 \\
13.1291\end{array}$ & $\begin{array}{l}\mathrm{y} \\
\mathrm{y}\end{array}$ & $\begin{array}{l}2 \\
1\end{array}$ & $\begin{array}{l}278.9 \\
279.6\end{array}$ & $\begin{array}{l}0.0 \\
0.2\end{array}$ & $\begin{array}{l}0.2376 \\
0.2440\end{array}$ & $\begin{array}{l}0.0 \\
0.2\end{array}$ & $\begin{array}{l}2.3 \\
2.2\end{array}$ & & & \\
\hline $13147-6335$ & $\mathrm{MLO} 3 \mathrm{Aa}, \mathrm{Ba}$ & HIP 64624 & 13.1291 & $\mathrm{y}$ & 1 & 38.9 & 0.0 & 1.6938 & 0.0 & 2.7 & & & \\
\hline $13147-6335$ & $\mathrm{MLO} 3 \mathrm{Ba}, \mathrm{Bb}$ & HIP 64624 & 13.1291 & $\mathrm{y}$ & 2 & 88.0 & 5.0 & 0.322 & 5.0 & $3.3:$ & & & \\
\hline $13149-1122$ & RST3829Aa,Ab & HIP 64638 & $\begin{array}{l}13.1290 \\
-1317\end{array}$ & $\mathrm{y}$ & 2 & 154.3 & 0.3 & 0.5678 & 0.1 & $1.8 *$ & 0.6 & -0.004 & Table 7 \\
\hline $\begin{array}{l}13169-3436 \\
13175-4033\end{array}$ & $\begin{array}{l}\text { I } 1567 \\
\text { I } 425\end{array}$ & $\begin{array}{l}\text { HIP } 64804 \\
\text { HIP } 64835\end{array}$ & $\begin{array}{l}13.1317 \\
13.1317\end{array}$ & $\mathrm{y}$ & 2 & 130.4 & 0.2 & 0.2895 & 0.1 & 1.2. & -3.0 & 0.050 & (Heintz 1986a) \\
\hline $\begin{array}{l}13175-4033 \\
13226-6059\end{array}$ & $\begin{array}{l}\text { I } 425 \\
\text { FIN } 208 \mathrm{AB}\end{array}$ & $\begin{array}{l}\text { HIP } 64835 \\
\text { HIP } 65271\end{array}$ & $\begin{array}{l}13.1317 \\
13.2383\end{array}$ & $\begin{array}{l}\mathrm{y} \\
\mathrm{y}\end{array}$ & $\begin{array}{l}2 \\
3\end{array}$ & $\begin{array}{l}354.1 \\
160.0\end{array}$ & $\begin{array}{l}0.3 \\
1.7\end{array}$ & $\begin{array}{l}0.2504 \\
0.0510\end{array}$ & $\begin{array}{l}0.3 \\
3.0\end{array}$ & $\begin{array}{l}1.2 \\
2.2\end{array}$ & & & \\
\hline $13254-5947$ & WSI 76 & $\begin{array}{l}\text { HIP } 65271 \\
\text { HIP } 65492\end{array}$ & 13.1291 & y & 1 & $\begin{array}{l}160.0 \\
187.7\end{array}$ & $\begin{array}{l}1.7 \\
2.1\end{array}$ & 0.1023 & $\begin{array}{l}3.0 \\
2.1\end{array}$ & 3.5 & & & \\
\hline $13310-3924$ & SEE 179 & HIP 65936 & 13.1317 & $\mathrm{y}$ & 2 & 55.7 & 0.0 & 0.1789 & 0.0 & 0.4 & 13.5 & -0.005 & (Finsen 1964) \\
\hline $\begin{array}{l}13317-0219 \\
13320-6519\end{array}$ & $\begin{array}{l}\text { HDS } 1895 \\
\text { FIN } 369\end{array}$ & $\begin{array}{l}\text { HIP } 65982 \\
\text { HIP } 66005\end{array}$ & $\begin{array}{l}13.1291 \\
13.1291\end{array}$ & $\begin{array}{l}\mathrm{y} \\
\mathrm{y}\end{array}$ & 2 & $\begin{array}{l}174.4 \\
333.5\end{array}-\longrightarrow$ & $\begin{array}{l}0.1 \\
0.1\end{array}$ & 0.1037 & 0.3 & 1.5 & $\begin{array}{l}1.9 \\
0.7\end{array}$ & -0.002 & (Hartkopf et al. 2012) \\
\hline $\begin{array}{l}1320-6519 \\
13325-6914\end{array}$ & $\begin{array}{ll}\text { I } 298 & 369 \\
\text { IN }\end{array}$ & $\begin{array}{l}\text { HIP } 66005 \\
\text { HIP } 66057\end{array}$ & $\begin{array}{l}13.1291 \\
13.2383\end{array}$ & $\stackrel{\mathrm{y}}{\mathrm{y}}$ & ${ }_{2}^{2}$ & $\begin{array}{l}333.5 \\
156.3\end{array}$ & $\begin{array}{l}0.1 \\
0.5\end{array}$ & 0.5316 & $\begin{array}{l}0.5 \\
0.5\end{array}$ & $\begin{array}{l}0.5 \\
1.4\end{array}$ & 3.0 & 0.039 & (Seymour et al. 2002) \\
\hline $13372-6142$ & I $365 \mathrm{AB}$ & HIP 66438 & 13.1291 & $\mathrm{y}$ & 2 & 183.4 & 0.0 & 0.3385 & 0.1 & $\begin{array}{l}1.4 \\
0.6\end{array}$ & -0.8 & -0.008 & (Söderhjelm 1999) \\
\hline $13437-4204$ & FIN $353 \mathrm{AB}$ & HIP 66984 & 13.1317 & $\mathrm{y}$ & 2 & 51.6 & 1.1 & 0.0642 & 0.4 & 1.0 & & & \\
\hline $13437-4204$ & RST1741AC & HIP 66984 & & & 2 & & 0.9 & 0.9521 & 0.7 & 2.8 & & & \\
\hline $13488-5250$ & RST2871 & HIP 67401 & 13.1291 & I & 2 & 129.2 & 0.8 & 2.0278 & 0.8 & $3.9 *$ & & & \\
\hline & & & 13.1318 & I & 2 & 129.1 & 0.6 & 2.0263 & 0.6 & $3.9 *$ & & & \\
\hline $13501-4451$ & DON $624 \mathrm{AB}$ & HIP 67527 & 13.1317 & $\mathrm{y}$ & $\frac{1}{2}$ & 116.6 & 0.8 & 1.0517 & 0.8 & $2.5 *$ & & & \\
\hline $13513-2423$ & WSI 77 & HIP 67620 & $\begin{array}{l}13.1317 \\
13.1317\end{array}$ & $\begin{array}{l}\mathrm{I} \\
\mathrm{y}\end{array}$ & $\begin{array}{l}2 \\
2\end{array}$ & $\begin{array}{r}116.6 \\
5.2\end{array}$ & $\begin{array}{l}0.3 \\
1.1\end{array}$ & $\begin{array}{l}1.00507 \\
0.1577\end{array}$ & $\begin{array}{l}0.5 \\
1.1\end{array}$ & $\begin{array}{l}3.1{ }^{*} \\
3.5\end{array}$ & -0.7 & 0.010 & (Tokovinin 2012) \\
\hline & & & 13.1317 & I & 2 & 4.6 & 0.9 & 0.1566 & 2.1 & 3.0 & -1.3 & 0.009 & \\
\hline $13520-3137$ & BU 343 & HIP 67696 & 13.1317 & $\mathrm{y}$ & 2 & 203.3 & 0.1 & 0.6356 & 0.1 & $1.1 *$ & 0.1 & -0.018 & Table 7 \\
\hline $13527-1843$ & WSI 78 & HIP 67744 & 13.1317 & $\mathrm{y}$ & 3 & 220.6 & 3.0 & 0.0234 & 3.8 & 1.2 & -51.8 & 0.011 & (Hartkopf et al. 2012) \\
\hline $\begin{array}{l}13539-1910 \\
13574-6229\end{array}$ & $\begin{array}{l}\text { HU } 898 \\
\text { FIN } 370\end{array}$ & $\begin{array}{l}\text { HIP } 67859 \\
\text { HIP } 68170\end{array}$ & $\begin{array}{l}13.1317 \\
13.1291\end{array}$ & $\begin{array}{l}\mathrm{y} \\
\mathrm{y}\end{array}$ & $\begin{array}{l}2 \\
2\end{array}$ & $\begin{array}{l}297.1 \\
111.7\end{array}$ & $\begin{array}{l}0.1 \\
0.1\end{array}$ & $\begin{array}{l}0.3499 \\
0.1552\end{array}$ & $\begin{array}{l}0.1 \\
0.1\end{array}$ & $\begin{array}{l}0.6 \\
0.4\end{array}$ & $\begin{array}{r}-0.4 \\
3.7\end{array}$ & $\begin{array}{r}0.001 \\
-0.001\end{array}$ & (Docoo \& Campo 2012) \\
\hline $\begin{array}{l}13574-6229 \\
14020-2108\end{array}$ & $\begin{array}{l}\text { FIN } 370 \\
\text { WSI } 79\end{array}$ & $\begin{array}{l}\text { HIP } 68170 \\
\text { HIP } 68552\end{array}$ & $\begin{array}{l}13.1291 \\
13.1318\end{array}$ & I & ${ }_{2}^{2}$ & $\begin{array}{l}111.7 \\
120.7\end{array}$ & $\begin{array}{l}0.1 \\
0.6\end{array}$ & $\begin{array}{l}0.1552 \\
0.1534\end{array}$ & $\begin{array}{l}0.1 \\
0.4\end{array}$ & $\begin{array}{l}0.4 \\
1.6\end{array}$ & 3.7 & -0.001 & (Mason et al. 2010) \\
\hline $14038-6022$ & $\mathrm{RBT} 1 \mathrm{Aa}, \mathrm{Ab}$ & HIP 68702 & 13.1291 & $\mathrm{y}$ & 2 & 316.2 & 6.2 & 0.0258 & 1.8 & 0.3 & 186.1 & -0.004 & (Davis et al. 2005) \\
\hline $14038-6022$ & VOU $31 \mathrm{Aa}, \mathrm{B}$ & HIP 68702 & 13.1291 & $\mathrm{y}$ & 2 & 200.5 & 2.9 & 0.4094 & 0.0 & 2. & & & \\
\hline $14152-6739$ & DON 652 & HIP 69643 & 13.1292 & $\mathrm{y}$ & 1 & 275.5 & 1.6 & 0.3802 & 1.6 & 3.5 & & & \\
\hline & & & 13.12 & I & 2 & 275.8 & 2.4 & 0.3 & 2.5 & 2.8 & & & \\
\hline $\begin{array}{l}14295-3702 \\
14330-4224\end{array}$ & $\begin{array}{l}\text { HDS2045Aa, Ab } \\
\text { HDS2054 }\end{array}$ & $\begin{array}{l}\text { HIP } 70868 \\
\text { HIP } 71140\end{array}$ & $\begin{array}{l}13.1318 \\
13.1318\end{array}$ & $\mathrm{y}$ & ${ }_{2}^{2}$ & & $\begin{array}{l}0.0 \\
0.0\end{array}$ & 0.0 & $\begin{array}{l}1.0 \\
0.1\end{array}$ & $\begin{array}{l}0 . \\
0 .\end{array}$ & & & \\
\hline $\begin{array}{l}4224 \\
4608\end{array}$ & $\begin{array}{l}\text { HDS26 } \\
\text { FIN } 31\end{array}$ & $\begin{array}{l}\text { HIP } 711 \\
\text { HIP } 715\end{array}$ & $\begin{array}{l}13.1318 \\
13.1292\end{array}$ & $\begin{array}{l}\mathrm{y} \\
\mathrm{y}\end{array}$ & $\begin{array}{l}2 \\
2\end{array}$ & $\begin{array}{l}233.6 \\
128.0\end{array}$ & $\begin{array}{l}0.0 \\
0.1\end{array}$ & $\begin{array}{l}0.0435 \\
0.1915\end{array}$ & $\begin{array}{l}0.1 \\
0.1\end{array}$ & $\begin{array}{l}0.4 \\
0.2\end{array}$ & 180.2 & 0.003 & Table 7 \\
\hline $\begin{array}{l}-49054 \\
-4954\end{array}$ & FIN 371 & HIP 71577 & $\begin{array}{l}13.12 \\
13.12\end{array}$ & $\mathrm{y}$ & 2 & $\begin{array}{r}128.0 \\
50.0\end{array}$ & $\begin{array}{l}0.1 \\
0.2\end{array}$ & $\begin{array}{l}0.1915 \\
0.0784\end{array}$ & $\begin{array}{l}0.1 \\
0.1\end{array}$ & $\begin{array}{l}0.2 \\
1.0\end{array}$ & & & Tabie $r$ \\
\hline 144 & I $528 \mathrm{AB}$ & HIP 721 & 13.1 & $\mathrm{y}$ & 2 & 28 & 0.1 & 0.06 & 0.1 & 1.5 & & & \\
\hline $14494-6714$ & DON 680 & HIP 72493 & 13.1292 & I & & 243.0 & 0.1 & 2.2366 & 1.2 & $1.5 *$ & & & \\
\hline $\begin{array}{l}14531-4638 \\
14567-6247\end{array}$ & $\begin{array}{l}\text { I } 952 \\
\text { FIN } 372 \\
-1\end{array}$ & $\begin{array}{l}\text { HIP } 72821 \\
\text { HIP } 73129\end{array}$ & $\begin{array}{l}13.1292 \\
13.1292\end{array}$ & $\mathrm{y}$ & ${ }_{2}^{2}$ & $\begin{array}{l}319.6 \\
341.5\end{array}$ & $\begin{array}{l}0.2 \\
0.0\end{array}$ & $\begin{array}{l}0.7284 \\
0.0987\end{array}$ & $\begin{array}{l}0.3 \\
0.3\end{array}$ & $\begin{array}{l}1.5{ }^{*} \\
0.4\end{array}$ & $\begin{array}{l}-0.0 \\
-2.9\end{array}$ & $\begin{array}{r}0.004 \\
-0.003\end{array}$ & $\begin{array}{l}\text { (Rica Romero \& Zirm 2011) } \\
\text { Rason et al 2010) }\end{array}$ \\
\hline $\begin{array}{l}14567-6247 \\
14575-2125\end{array}$ & $\mathrm{H} \mathrm{N} 28 \mathrm{Ba}, \mathrm{Bb}$ & $\begin{array}{l}\text { HIP } 73182 \\
\text { HIP }\end{array}$ & $\begin{array}{l}13.1292 \\
13.1318\end{array}$ & $\begin{array}{l}\mathrm{y} \\
\mathrm{y}\end{array}$ & $\frac{2}{2}$ & $\begin{array}{l}341.5 \\
209.2\end{array}$ & $\begin{array}{l}0.0 \\
0.1\end{array}$ & $\begin{array}{l}0.0987 \\
0.1933\end{array}$ & $\begin{array}{l}0.3 \\
0.3\end{array}$ & $\begin{array}{l}0.4 \\
1.9\end{array}$ & $\begin{array}{l}-2.9 \\
-1.0\end{array}$ & $\begin{array}{l}-0.003 \\
-0.002\end{array}$ & $\begin{array}{l}\text { (Mason et al. } \\
\text { (Forveille et al. } 1910) \\
\end{array}$ \\
\hline $14581-4852$ & WSI 80 & HIP 73241 & 13.1292 & $\mathrm{y}$ & 2 & 113.7 & 0.7 & 0.3872 & 2.9 & 4.5 & -1.0 & & 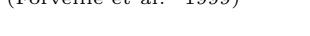 \\
\hline $15047-5625$ & RST2937 & HD 133044 & $\begin{array}{l}13.1292 \\
13.1319\end{array}$ & $\mathrm{I}$ & ${ }_{2}^{2}$ & $\begin{array}{l}113.6 \\
156.4\end{array}$ & 1.2 & 0.3864 & $\begin{array}{l}1.1 \\
0.3\end{array}$ & 3.2 & & & \\
\hline
\end{tabular}


TABLE 2-Continued

\begin{tabular}{|c|c|c|c|c|c|c|c|c|c|c|c|c|c|}
\hline $\begin{array}{l}\text { WDS } \\
(2000)\end{array}$ & $\begin{array}{c}\text { Discoverer } \\
\text { Designation } \\
\end{array}$ & $\begin{array}{l}\text { Other } \\
\text { name }\end{array}$ & $\begin{array}{l}\text { Epoch } \\
+2000 \\
\end{array}$ & $\begin{array}{c}\text { Filt } \\
\end{array}$ & $\mathrm{N}$ & $\begin{array}{c}\theta \\
(\mathrm{deg})\end{array}$ & $\begin{array}{l}\rho \sigma_{\theta} \\
(\mathrm{mas})\end{array}$ & $\begin{array}{l}\rho \\
\left({ }^{\prime \prime}\right)\end{array}$ & $\begin{array}{c}\sigma \rho \\
\text { (mas) }\end{array}$ & $\begin{array}{l}\Delta m \\
(\mathrm{mag})\end{array}$ & $\begin{array}{c}{[\mathrm{O}-\mathrm{C}]_{\theta}} \\
(\mathrm{deg})\end{array}$ & $\begin{array}{c}{\left[\begin{array}{l}\mathrm{O}-\mathrm{C}]_{\rho} \\
\left({ }^{\prime \prime}\right)\end{array}\right.} \\
\end{array}$ & $\begin{array}{c}\begin{array}{c}\text { Reference } \\
\text { code* }\end{array} \\
\end{array}$ \\
\hline $15143-4242$ & WSI $82 \mathrm{Aa}, \mathrm{Ab}$ & HD 135132 & 13.1318 & $\mathrm{y}$ & 1 & 56.9 & 0.0 & 0.0450 & 0.0 & 1.7 & & & \\
\hline & & & & $\mathrm{I}$ & 2 & & 0.1 & & & & & & \\
\hline $15143-4242$ & B $1273 \mathrm{Aa}, \mathrm{B}$ & HD 135132 & 13.1318 & y & 1 & 132.2 & 0.0 & 0.5650 & 0.0 & 0.9 & & & \\
\hline $15246-4835$ & B $1288 \mathrm{~A} \mathrm{~B}$ & HIP 75427 & $\begin{array}{l}13.1318 \\
13.1319\end{array}$ & I & $\begin{array}{r}2 \\
2\end{array}$ & $\begin{array}{r}131.8 \\
78.4\end{array}$ & $\begin{array}{l}1.6 \\
0.5\end{array}$ & $\begin{array}{l}0.5623 \\
0.0355\end{array}$ & 1.7 & 0.7 & 670 & -0.024 & (Seymour et al. 2002) \\
\hline $15251-3810$ & RST2955 & HIP 75476 & 13.1318 & y & 2 & 215.5 & $\begin{array}{l}0.0 \\
0.1\end{array}$ & 0.0826 & 0.1 & $\begin{array}{l}0.3 \\
0.3\end{array}$ & 67.0 & -0.024 & Deymour el al. 2002 \\
\hline $15339-1700$ & HDS2185 & HIP 76203 & 13.1319 & y & 2 & 139.6 & 0.2 & 0.3541 & 0.2 & 1.8 & & & \\
\hline $15348-2808$ & TOK $49 \mathrm{Aa}, \mathrm{Ab}$ & HIP 76275 & 13.1319 & $\mathrm{y}$ & 2 & 239.8 & 2.5 & 0.1155 & 3.2 & 2.6 & & & \\
\hline $\begin{array}{l}15348-2808 \\
15355-4751\end{array}$ & $\begin{array}{l}\text { RST1847Aa,B } \\
\text { HDS2191 }\end{array}$ & $\begin{array}{l}\text { HIP } 76275 \\
\text { HIP } 76328\end{array}$ & $\begin{array}{l}13.1319 \\
13.1319\end{array}$ & y & 2 & 335.8 & 0.5 & 0.9517 & 2.5 & 2.6 & & & \\
\hline $\begin{array}{l}155355-4751 \\
15451-3506\end{array}$ & $\begin{array}{l}\text { HDS2191 } \\
\text { SEE 248 }\end{array}$ & $\begin{array}{l}\text { HIP } 76328 \\
\text { HIP } 77150\end{array}$ & $\begin{array}{l}13.1319 \\
13.1319\end{array}$ & $\begin{array}{l}\mathrm{y} \\
\mathrm{y}\end{array}$ & $\begin{array}{l}2 \\
2\end{array}$ & $\begin{array}{l}201.4 \\
348.0\end{array}$ & $\begin{array}{l}0.3 \\
0.5\end{array}$ & $\begin{array}{l}0.2480 \\
0.1481\end{array}$ & $\begin{array}{l}0.3 \\
0.5\end{array}$ & $\begin{array}{l}1.0 \\
3.8\end{array}$ & & & \\
\hline & & & 13.1319 & I & 2 & $\begin{array}{l}348.0 \\
348.5\end{array}$ & $\begin{array}{l}0.5 \\
1.0\end{array}$ & 0.1443 & $\begin{array}{l}0.5 \\
1.8\end{array}$ & $\begin{array}{l}3.8 \\
2.7\end{array}$ & & & \\
\hline $15462-2804$ & BU $620 \mathrm{AB}$ & HIP 77235 & 13.1319 & $\mathrm{y}$ & 2 & 353.6 & 0.0 & 0.6334 & 0.1 & 0.7 & & & \\
\hline $15471-5107$ & B $1790 \mathrm{~A}, \mathrm{Ba}$ & HD 140662 & $\begin{array}{l}13.1319 \\
13.1319\end{array}$ & y & 2 & $\begin{array}{l}83.3 \\
83.6\end{array}$ & 2.8 & 0.4258 & 3.3 & 0.9 & & & \\
\hline $15471-5107$ & & HD 140662 & $\begin{array}{l}13.1319 \\
13.1319\end{array}$ & y & $\begin{array}{l}1 \\
2\end{array}$ & $\begin{array}{r}83.6 \\
221.1\end{array}$ & $\begin{array}{l}0.0 \\
0.1\end{array}$ & $\begin{array}{l}0.4276 \\
0.0718\end{array}$ & $\begin{array}{l}0.0 \\
4.5\end{array}$ & $\begin{array}{l}0.5 \\
0.3\end{array}$ & & & \\
\hline $10461-0106$ & WS1 78Ba,Bb & HD 140662 & 13.1319 & I & 1 & 223.6 & $\begin{array}{l}0.1 \\
0.0\end{array}$ & $\begin{array}{l}0.0718 \\
0.0719\end{array}$ & $\begin{array}{l}4.5 \\
0.0\end{array}$ & 0.3 & & & \\
\hline $15549-3731$ & B 852 & HIP 77931 & 13.1319 & $\mathrm{y}$ & 1 & 255.4 & 1.5 & 0.9716 & 1.5 & $2.8 *$ & & & \\
\hline & & & & $\mathrm{I}$ & 2 & 255.1 & 0.4 & 0.9725 & 0.4 & $3.8 *$ & & & \\
\hline $17018-5108$ & I 1306 & HIP 83321 & 13.7469 & $\mathrm{y}$ & 2 & 195.9 & 0.2 & 0.2401 & 0.1 & 0.4 & -4.4 & 0.115 & (Olevic \& Cvetkovic 2004a) \\
\hline $17031-5314$ & $\mathrm{HDS} 2412 \mathrm{Aa}, \mathrm{Ab}$ & HIP 83431 & 13.7469 & y & 2 & 182.4 & 0.6 & 0.6345 & 0.4 & 3.5 & & & \\
\hline & & & $\begin{array}{r}13.7469 \\
13.4736\end{array}$ & I & 2 & 182.3 & 0.5 & 0.6340 & 0.3 & 2.8 & & & \\
\hline $17066+0039$ & $\mathrm{BU} 823 \mathrm{~A}, \mathrm{Ba}$ & HIP 83716 & $\begin{array}{l}13.4736 \\
13.4736\end{array}$ & $\stackrel{\mathrm{y}}{\mathrm{I}}$ & $\begin{array}{l}1 \\
3\end{array}$ & $\begin{array}{l}167.1 \\
167.1\end{array}$ & $\begin{array}{l}0.0 \\
30\end{array}$ & $\begin{array}{l}1.0220 \\
1.0240\end{array}$ & $\begin{array}{l}0.0 \\
1.0\end{array}$ & $\begin{array}{l}2.1 \\
1.5\end{array}$ & $\begin{array}{l}-3.0 \\
-3.0\end{array}$ & $\begin{array}{l}0.003 \\
0.005\end{array}$ & (Hartkopf \& Mason 2000) \\
\hline & & & $\begin{array}{l}13.4736 \\
13.4736\end{array}$ & y & $\begin{array}{l}3 \\
1\end{array}$ & $\begin{array}{l}167.1 \\
252.3\end{array}$ & $\begin{array}{l}3.0 \\
0.0\end{array}$ & $\begin{array}{l}1.0240 \\
0.0454\end{array}-100$ & $\begin{array}{l}1.0 \\
0.0\end{array}$ & $\begin{array}{l}1.5 \\
0.1\end{array}$ & $\begin{array}{l}-3.0 \\
-0.7\end{array}$ & $\begin{array}{r}0.005 \\
-0.003\end{array}$ & Table 6 \\
\hline $17000+0009$ & HON JZDa,DD & П11F 80,10 & 13.4736 & I & 3 & 255.0 & 0.6 & 0.0460 & 3.4 & 0.1 & $\begin{array}{r}-0.0 \\
2.0\end{array}$ & $\begin{array}{l}-0.000 \\
-0.000\end{array}$ & Table o \\
\hline $17119-0151$ & LPM 629 & HIP 84123 & 13.4737 & $\mathrm{I}$ & 2 & 246.6 & 0.1 & 0.7697 & 0.3 & $0.1 *$ & -14.9 & -0.061 & (Söderhjelm 1999) \\
\hline $17130+0745$ & STT 325 & HIP 84230 & 13.4736 & & 2 & 293.3 & 0.2 & 0.3598 & 0.4 & 2.0 & 0.3 & 0.009 & (Rica Romero 201 \\
\hline $17141-0824$ & BAR 7 & HIP 84303 & 13.4737 & $\mathrm{I}$ & 2 & 50.0 & 0.8 & 1.4879 & 0.7 & $2.1 *$ & -5.2 & -0.131 & (Cvetkovic et al. 2008) \\
\hline $17156-1018$ & BU 957 & HIP 84423 & 13.4737 & $\mathrm{y}$ & 2 & 164.7 & 0.1 & 0.0887 & 0.5 & 0.3 & -21.0 & -0.187 & (Heintz 1984) \\
\hline $17157-0949$ & A $2592 \mathrm{~A}, \mathrm{Ba}$ & HIP 84430 & 13.4737 & y & 1 & 120.6 & 0.0 & 0.1617 & 0.0 & 1.1 & 1.7 & -0.004 & Table 7 \\
\hline & & & $\begin{array}{l}13.4737 \\
13.7470\end{array}$ & I & $\begin{array}{r}2 \\
2\end{array}$ & $\begin{array}{l}120.1 \\
117.2\end{array}$ & 0.6 & $\begin{array}{l}0.1630 \\
0.1636\end{array}$ & $\begin{array}{l}0.0 \\
0.3\end{array}$ & $\begin{array}{l}1.5 \\
1.4\end{array}$ & 1.2 & $\begin{array}{l}-0.003 \\
-0.001\end{array}$ & \\
\hline $17157-0949$ & TOK $53 \mathrm{Ba}, \mathrm{Bb}$ & HIP 84430 & 13.4737 & $\begin{array}{l}\mathrm{y} \\
\mathrm{y}\end{array}$ & $\frac{2}{1}$ & $\begin{array}{l}117.2 \\
174.8\end{array}$ & $\begin{array}{l}2.6 \\
0.0\end{array}$ & $\begin{array}{l}0.1636 \\
0.0427\end{array}$ & $\begin{array}{l}0.3 \\
0.0\end{array}$ & $\begin{array}{l}1.4 \\
0.4\end{array}$ & & & \\
\hline & 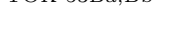 & N11F 84450 & 13.4737 & I & 2 & 177.3 & 0.4 & 0.0406 & 0.3 & $\begin{array}{l}0.4 \\
0.4\end{array}$ & & & \\
\hline & & & $\begin{array}{l}13.7470 \\
13.7469\end{array}$ & $\mathrm{y}$ & 2 & 175.1 & 1.3 & 0.0387 & 0.3 & 0.2 & & & \\
\hline $17195-5004$ & FIN 356 & $\begin{array}{l}\text { HIP } 84759 \\
\text { HIP } 84866\end{array}$ & $\begin{array}{l}13.7469 \\
13.4737\end{array}$ & $\mathrm{y}$ & ${ }^{2}$ & $\begin{array}{r}237.9 \\
3470\end{array}$ & 0.0 & 0.0494 & 0.2 & 0.6 & 184.9 & $\begin{array}{l}0.001 \\
0.023\end{array}$ & $\begin{array}{l}\text { Table } 6 \\
\text { Hejintz }\end{array}$ \\
\hline $17240-0921$ & RST3972Aa, Ab & HIP 85141 & 13.4737 & y & $\frac{2}{2}$ & $\begin{array}{l}341.0 \\
263.4\end{array}$ & 0.4 & 0.2272 & 0.6 & $\begin{array}{l}0.6 \\
0.6\end{array}$ & -2.3 & -0.002 & (Soderhjelm 1999) \\
\hline $17248-5913$ & I $385 \mathrm{AB}$ & HIP 85216 & 13.7469 & $\mathrm{y}$ & 2 & 119.7 & 0.3 & 0.3992 & 5.8 & 1.7 & & & \\
\hline $\begin{array}{l}17248-5913 \\
17305-1006\end{array}$ & $\begin{array}{l}\text { WSI 87AD } \\
\text { RST3978 }\end{array}$ & HIP 85216 & $\begin{array}{l}13.7469 \\
134737\end{array}$ & $\mathrm{y}$ & $\begin{array}{r}2 \\
2\end{array}$ & $\begin{array}{r}271.9 \\
79.4\end{array}$ & $\begin{array}{l}2.7 \\
0.4\end{array}$ & 0.2702 & 0.1 & 1.6 & & & \\
\hline $17305-1006$ & RST3978 & HIP 85675 & $\begin{array}{l}13.4737 \\
13.7470\end{array}$ & $\begin{array}{l}\mathrm{y} \\
\mathrm{y}\end{array}$ & ${ }_{2}^{2}$ & $\begin{array}{l}79.4 \\
79.5\end{array}$ & $\begin{array}{l}0.4 \\
0.3\end{array}$ & $\begin{array}{l}0.2518 \\
0.2628\end{array}-1$ & $\begin{array}{l}0.7 \\
0.3\end{array}$ & $\begin{array}{l}2.3 \\
2.4\end{array}$ & & & \\
\hline $17390+0240$ & WSI 88 & HIP 86374 & 13.4736 & y & 2 & 8.7 & 0.4 & 0.1845 & 0.4 & 2.7 & & & \\
\hline 5348 & HDS2502 & HIP 86569 & 13.7469 & $\mathrm{I}$ & 2 & 157.4 & 0.3 & 0.1601 & 1.2 & 0.4 & & & \\
\hline $17461+0532$ & CHR 157 & HIP 86954 & 13.4736 & $\mathrm{y}$ & 2 & 34.5 & 1.4 & 0.3098 & 1.0 & 3.2 & & & \\
\hline $17535-0355$ & TOK 54 & $\mathrm{~V} 2610 \mathrm{Oph}$ & 13.4737 & I & 2 & 129.4 & 0.2 & 0.1161 & 1.1 & 0.5 & & & \\
\hline $17575-5740$ & $\mathrm{HJ} 4992 \mathrm{~A}, \mathrm{Ba}$ & HIP 87914 & 13.7469 & $\mathrm{y}$ & 2 & 42.1 & 0.6 & 2.5203 & 7.8 & 3.9 & & & \\
\hline & & & 13.7469 & I & 2 & 42.1 & 0.7 & 2.5136 & 1.3 & 1.4 & & & \\
\hline $17575-5740$ & TOK $55 \mathrm{Ba}, \mathrm{Bb}$ & HIP 87914 & $\begin{array}{r}13.7469 \\
13.7469\end{array}$ & $\mathrm{I}_{\mathrm{I}}^{\mathrm{y}}$ & 2 & 141.2 & $\begin{array}{l}7.9 \\
6.1\end{array}$ & 0.0572 & $\begin{array}{l}1.4 \\
0.5\end{array}$ & 0.4 & & & \\
\hline $18092-2211$ & RST3157 & HIP 88932 & $\begin{array}{l}13.7469 \\
13.7470\end{array}$ & y & $\frac{2}{2}$ & $\begin{array}{l}144.3 \\
209.2\end{array}$ & $\begin{array}{l}6.1 \\
0.1\end{array}$ & 0.1156 & $\begin{array}{l}0.5 \\
0.4\end{array}$ & $\begin{array}{l}0.1 \\
1.1\end{array}$ & 3.1 & 0.004 & (Heintz 1990) \\
\hline $18112-1951$ & $\mathrm{BU} 132 \mathrm{AB}$ & HIP 89114 & 13.4737 & y & 2 & 188.8 & 0.4 & 1.4167 & 0.6 & 0.9 & 1.2 & 0.019 & (Hartkopf \& Mason 2011) \\
\hline & & & $\begin{array}{l}13.7470 \\
13.7469\end{array}$ & y & ${ }^{2}$ & 188.2 & 0.1 & 1.4182 & 1.1 & $1.8 *$ & 0.7 & 0.020 & \\
\hline $18126-7340$ & TOK $58 \mathrm{Aa}, \mathrm{Ab}$ & HIP 89234 & $\begin{array}{l}13.7469 \\
13.7469\end{array}$ & $\begin{array}{l}\mathrm{I} \\
\mathrm{y}\end{array}$ & $\begin{array}{l}3 \\
2\end{array}$ & $\begin{array}{l}301.4 \\
303.4\end{array}$ & $\begin{array}{l}2.0 \\
3.8\end{array}$ & $\begin{array}{l}0.1346 \\
0.1300\end{array}$ & $\begin{array}{l}4.1 \\
3.2\end{array}$ & $\begin{array}{l}3.3 \\
4.4\end{array}$ & & & \\
\hline $18126-7340$ & $\mathrm{HDO} 284 \mathrm{Aa}, \mathrm{B}$ & HIP 89234 & 13.7469 & I & 3 & 270.1 & 5.4 & 2.1804 & 4.9 & 4.0 & & & \\
\hline & & & $\begin{array}{r}13.7469 \\
13.4737\end{array}$ & y & 2 & 270.0 & 5.4 & $\begin{array}{r}2.1835 \\
0.1972\end{array}$ & 12.7 & 6.1 & & & \\
\hline $\begin{array}{l}18141-1912 \\
18150-5018\end{array}$ & $\begin{array}{l}\mathrm{CHR} 234 \mathrm{Aa}, \mathrm{Ab} \\
\text { I } 429\end{array}$ & $\begin{array}{l}\text { HD } 167034 \\
\text { HD } 166839\end{array}$ & $\begin{array}{l}13.4737 \\
13.7469\end{array}$ & $\begin{array}{l}1 \\
y\end{array}$ & $\begin{array}{l}2 \\
2\end{array}$ & $\begin{array}{r}60.0 \\
342.4\end{array}$ & $\begin{array}{l}1.1 \\
1.1\end{array}$ & $\begin{array}{l}0.1972 \\
0.0329\end{array}$ & $\begin{array}{l}1.4 \\
0.7\end{array}$ & $\begin{array}{l}0.9 \\
0.6\end{array}$ & & & \\
\hline $18152-2023$ & $\mathrm{CHR} 255 \mathrm{Aa}, \mathrm{Ab}$ & HIP 89440 & 13.7 & y & 2 & 155.7 & 0.1 & 0.0 & 0.1 & 0.4 & & & \\
\hline $18152-2044$ & TOK 59 & HIP 89439 & 13.7470 & y & 2 & 76.6 & 1.4 & 1.2767 & 1.4 & 5.6 & & & \\
\hline $18171-4336$ & HDS2583 & HIP 89599 & $\begin{array}{l}13.7470 \\
13.7470\end{array}$ & I & $\begin{array}{l}2 \\
2\end{array}$ & $\begin{array}{l}76.6 \\
81.2\end{array}$ & $\begin{array}{l}2.0 \\
0.2\end{array}$ & $\begin{array}{l}1.2755 \\
0.0460\end{array}$ & $\begin{array}{l}2.0 \\
0.6\end{array}$ & $\begin{array}{l}5.4 \\
0.9\end{array}$ & & & \\
\hline $18171-4336$ & HDS2583 & HIP 89599 & 13.7470 & & & 81.2 & & 0.0460 & & & & & \\
\hline
\end{tabular}


TABLE 2-Continued

\begin{tabular}{|c|c|c|c|c|c|c|c|c|c|c|c|c|c|}
\hline $\begin{array}{l}\text { WDS } \\
(2000)\end{array}$ & $\begin{array}{c}\text { Discoverer } \\
\text { Designation }\end{array}$ & $\begin{array}{l}\text { Other } \\
\text { name }\end{array}$ & $\begin{array}{l}\text { Epoch } \\
+2000\end{array}$ & Filt & $\mathrm{N}$ & $\begin{array}{c}\theta \\
(\operatorname{deg})\end{array}$ & $\begin{array}{l}\rho \sigma_{\theta} \\
(\mathrm{mas})\end{array}$ & $\begin{array}{l}\rho \\
\left({ }^{\prime \prime}\right)\end{array}$ & $\begin{array}{c}\sigma \rho \\
(\mathrm{mas})\end{array}$ & $\begin{array}{l}\Delta m \\
(\mathrm{mag})\end{array}$ & $\begin{array}{c}{[\mathrm{O}-\mathrm{C}]_{\theta}} \\
(\mathrm{deg})\end{array}$ & $\begin{array}{c}{[\mathrm{O}-\mathrm{C}]_{\rho}} \\
\left({ }^{\prime \prime}\right)\end{array}$ & $\begin{array}{c}\text { Reference } \\
\text { code* }\end{array}$ \\
\hline \multirow[t]{2}{*}{$18177-1940$} & BU $246 \mathrm{~A}, \mathrm{Ba}$ & HIP 89647 & 13.4737 & $\mathrm{y}$ & 1 & 117.1 & 0.0 & 0.5193 & 0.0 & 1.3 & & & \\
\hline & & & $\begin{array}{l}13.4737 \\
13.7470\end{array}$ & I & $\frac{1}{2}$ & $\begin{array}{l}117.4 \\
116.2\end{array}$ & $\begin{array}{l}0.0 \\
0.3\end{array}$ & $\begin{array}{l}0.515 \\
0.5166\end{array}$ & $\begin{array}{l}0.0 \\
0.8\end{array}$ & 1.1 & & & \\
\hline \multirow{3}{*}{$18177-1940$} & WSI $89 \mathrm{Ba}, \mathrm{Bb}$ & HIP 89647 & $\begin{array}{l}13.7470 \\
13.4737\end{array}$ & $\begin{array}{l}\mathrm{y} \\
\mathrm{y}\end{array}$ & $\begin{array}{l}2 \\
1\end{array}$ & $\begin{array}{l}116.2 \\
347.3\end{array}$ & $\begin{array}{l}0.3 \\
0.0\end{array}$ & $\begin{array}{l}0.5166 \\
0.0475\end{array}$ & $\begin{array}{l}0.8 \\
0.0\end{array}$ & $\begin{array}{l}0.6 \\
0.9\end{array}$ & & & \\
\hline & & & & I & 1 & 353.2 & 0.0 & 0.0505 & 0.0 & $0.9:$ & & & \\
\hline & & & 13.7470 & $\mathrm{y}$ & 2 & 351.7 & 1.2 & 0.0554 & 1.0 & 1.1 & & & \\
\hline $18190-4759$ & B 936 & HIP 89752 & 13.7469 & I & 3 & 347.4 & 0.1 & 0.3040 & 0.2 & & & & \\
\hline $18250-0135$ & AC 11 & HIP 90253 & 13.4737 & $\mathrm{y}$ & 2 & 355.2 & 0.2 & 0.9077 & 0.7 & $0.4 *$ & 0.7 & 0.090 & (Heintz 1995) \\
\hline \multirow{2}{*}{$\begin{array}{l}18289-2503 \\
18323-1439\end{array}$} & $\begin{array}{l}\mathrm{H} \mathrm{N} 125 \\
\mathrm{CHR} 73\end{array}$ & HIP 90574 & $\begin{array}{l}13.4738 \\
13.4737\end{array}$ & $\mathrm{I}$ & ${ }_{2}^{2}$ & $\begin{array}{l}107.2 \\
226.2\end{array}$ & 1.3 & 2.5229 & 0.2 & $0.1 *$ & & 0.022 & (Oleyic \& Cyetkoyic 2005) \\
\hline & CHR 73 & HIP 90884 & 13.7471 & $\begin{array}{l}\mathrm{y} \\
\mathrm{y}\end{array}$ & ${ }_{2}^{2}$ & $\begin{array}{l}226.2 \\
218.6\end{array}$ & $\begin{array}{l}0.8 \\
0.8\end{array}$ & $\begin{array}{l}0.0560 \\
0.0587\end{array}$ & $\begin{array}{l}0.2 \\
0.1\end{array}$ & $\begin{array}{l}1.8 \\
1.7\end{array}$ & $\begin{array}{l}60.8 \\
87.1\end{array}$ & $\begin{array}{l}0.022 \\
0.025\end{array}$ & Olevic \& Cvetkovic 2005) \\
\hline \multirow{2}{*}{$18329-7358$} & HDS2631 & HIP 90930 & 13.7470 & y & 2 & 78.9 & 0.9 & 0.5395 & 0.9 & 4.4 & & & \\
\hline & & & 13.7470 & I & 2 & 79.0 & 3.1 & 0.5403 & 1.5 & 4.7 & & & \\
\hline $18331-1042$ & HDS2632 & HIP 90943 & 13.4737 & y & 1 & 18.8 & 3.6 & 0.5127 & 3.6 & 4.4 & & & \\
\hline \multirow{2}{*}{$18368-2617$} & RST3187 & HP 91253 & $\begin{array}{l}13.4737 \\
13.4738\end{array}$ & ${ }_{\mathrm{I}}^{\mathrm{I}}$ & ${ }_{2}^{2}$ & $\begin{array}{l}18.6 \\
85.4\end{array}$ & $\begin{array}{l}1.6 \\
0.3\end{array}$ & $\begin{array}{l}0.5123 \\
0.0779\end{array}$ & $\begin{array}{l}0.6 \\
1.4\end{array}$ & 2.8 & & & \\
\hline & RST3187 & HIP 91253 & $\begin{array}{l}13.4738 \\
13.7470\end{array}$ & $\begin{array}{l}1 \\
y\end{array}$ & $\begin{array}{l}2 \\
2\end{array}$ & $\begin{array}{l}8.4 \\
92.8\end{array}$ & $\begin{array}{l}0.3 \\
0.2\end{array}$ & $\begin{array}{l}0.0779 \\
0.0685\end{array}$ & $\begin{array}{l}1.4 \\
0.1\end{array}$ & $\begin{array}{l}0.3 \\
1.0\end{array}$ & & & \\
\hline $18434-5546$ & B $398 \mathrm{AB}$ & HIP 91837 & 13.7469 & y & 2 & 3.5 & 0.2 & 0.1675 & 0.3 & 1.5 & -39.2 & 0.088 & (Heintz 1978b) \\
\hline $18439-0649$ & $\begin{array}{l}\text { YSC } 133 \\
\text { CHB } 78\end{array}$ & HIP 91880 & 13.7471 & & 2 & 259.2 & 0.1 & 0.0593 & 0.3 & 0.7 & & & \\
\hline $\begin{array}{l}18448-2501 \\
18448-3323\end{array}$ & $\begin{array}{l}\text { CHR } 78 \\
\text { OL } 20\end{array}$ & $\begin{array}{l}\text { HIP } 91974 \\
\text { HIP } 91978\end{array}$ & $\begin{array}{l}13.7470 \\
13.7470\end{array}$ & y & 2 & $\begin{array}{l}191.9 \\
2590\end{array}$ & 1.4 & $\begin{array}{l}0.0532 \\
0.1145\end{array}$ & 1.1 & $\begin{array}{l}3.1 \\
0.8\end{array}$ & & & \\
\hline $\begin{array}{l}18448-3323 \\
18480-1009\end{array}$ & $\begin{array}{l}\text { OL } 20 \\
\text { HDS2665 }\end{array}$ & $\begin{array}{l}\text { HIP } 91978 \\
\text { HIP } 92250\end{array}$ & $\begin{array}{l}13.7470 \\
13.4737\end{array}$ & ${ }_{\mathrm{I}}^{\mathrm{y}}$ & $\begin{array}{l}2 \\
2\end{array}$ & $\begin{array}{r}259.0 \\
81.2\end{array}$ & $\begin{array}{l}0.1 \\
0.5\end{array}$ & $\begin{array}{l}\begin{array}{l}0.1145 \\
0.3939\end{array} \\
0\end{array}$ & $\begin{array}{l}0.1 \\
0.7\end{array}$ & $\begin{array}{l}0.8 \\
2.2\end{array}$ & & & \\
\hline $18490-0828$ & $\begin{array}{l}\text { HDS2665 } \\
\text { HDS266a }, \mathrm{Ab}\end{array}$ & $\begin{array}{l}\text { HIP } 92250 \\
\text { HIP } 92322\end{array}$ & $\begin{array}{l}13.4737 \\
13.7471\end{array}$ & y & 1 & $\begin{aligned} 01.2 \\
257.7\end{aligned}$ & $\begin{array}{l}0.0 \\
0.1\end{array}$ & 0.1761 & 0.1 & 1.2 & & & \\
\hline $18516-6054$ & RST5126 & HIP 92547 & 13.7469 & y & $\frac{1}{2}$ & 243.2 & $\begin{array}{l}0.1 \\
0.3\end{array}$ & $\begin{array}{l}0.171 \\
0.0691\end{array}$ & $\begin{array}{l}0.1 \\
0.2\end{array}$ & 1.4 & & & \\
\hline $18520-5418$ & TOK $325 \mathrm{Aa}, \mathrm{Ab}$ & HIP 92592 & 13.7469 & y & 2 & 292.6 & 0.9 & 0.1232 & 1.5 & 2.9 & & & \\
\hline $18531-2745$ & I 1031 & HD 17472 & 13.7470 & $\mathrm{y}$ & 2 & 341.9 & 0.2 & 0.3865 & 0.1 & 1.8 & & & \\
\hline $18567-5102$ & RST5456 & HIP 92991 & 13.7469 & y & 2 & 322.3 & 0.2 & 0.1860 & 0.3 & 1.1 & & & \\
\hline $19026-2953$ & HDO 150AB & HIP 93506 & 13.4739 & y & 2 & 266.5 & 0.1 & 0.5045 & 0.1 & 0.4 & 1.6 & -0.001 & (DeRosa et al. 2012) \\
\hline $\begin{array}{l}19155-2515 \\
19253-2431\end{array}$ & B 430 & HIP 94643 & $\begin{array}{l}13.4739 \\
13.4739\end{array}$ & $\mathrm{y}$ & 2 & 271.2 & 0.1 & 0.0344 & 0.2 & 0.4 & -10.6 & -0.023 & (Hartkopf \& Mason 2001b) \\
\hline \multirow{4}{*}{$20104-1923$} & $\begin{array}{l}\text { FDN } 327 \\
\text { HDS } 2873\end{array}$ & $\begin{array}{l}\text { HIP } 95477 \\
\text { HIP } 99391\end{array}$ & $\begin{array}{l}13.4739 \\
12.9229\end{array}$ & I & ${ }_{2}^{4}$ & $\begin{array}{r}86.9 \\
210.2\end{array}$ & $\begin{array}{l}0.7 \\
1.7\end{array}$ & $\begin{array}{l}0.0522 \\
0.1183\end{array}$ & $\begin{array}{l}1.0 \\
3.5\end{array}$ & $\begin{array}{l}0.6 \\
2.5\end{array}$ & & & (Mason \& Hartkopt 1999) \\
\hline & & & 12.9229 & y & 2 & 207.9 & 1.5 & 0.1147 & $\begin{array}{l}3.0 \\
0.6\end{array}$ & 2.8 & & & \\
\hline & & & 13.7363 & I & 1 & 203.3 & 0.1 & 0.0862 & 0.1 & 2.2 & & & \\
\hline & & & 13.7363 & y & 2 & 206.4 & 0.7 & 0.0912 & 0.3 & 3.4 & & & \\
\hline $20111-5731$ & HDO 295 & HIP 99453 & 13.7362 & $\mathrm{y}$ & 2 & 287.0 & 0.1 & 0.3626 & 0.2 & 1.3 & & & \\
\hline \multirow{3}{*}{$20202-3435$} & HDS2899 & HIP 100065 & 13.7363 & y & 2 & 235.9 & 0.4 & & 0.6 & 3.0 & & & \\
\hline & I 1416 & HIP 100266 & $\begin{array}{r}12.9229 \\
13.7362\end{array}$ & $\mathrm{y}$ & 2 & 119.1 & 0.1 & 0.2324 & 0.3 & 1.2 & -51.3 & 0.216 & (van den Bos 1961) \\
\hline & & & $\begin{array}{l}13.7362 \\
13.7362\end{array}$ & $\begin{array}{l}1 \\
\text { y }\end{array}$ & $\begin{array}{l}2 \\
1\end{array}$ & $\begin{array}{l}118.1 \\
118.1\end{array}$ & $\begin{array}{l}0.2 \\
0.1\end{array}$ & $\begin{array}{l}0.2173 \\
0.2173\end{array}$ & $\begin{array}{l}0.2 \\
0.1\end{array}$ & $\begin{array}{l}1.1 \\
1.2\end{array}$ & $\begin{array}{l}-3.5 \\
-3.5\end{array}$ & $\begin{array}{l}0.175 \\
0.175\end{array}$ & \\
\hline \multirow[t]{2}{*}{$20217-3637$} & HDS2908 & HIP 100417 & 12.9229 & $\mathrm{y}$ & 2 & 102.4 & 0.2 & 0.1457 & 0.5 & 0.4 & & & \\
\hline & & & 12.9229 & I & 2 & 102.1 & 0.2 & 0.1453 & 0.1 & 0.2 & & & \\
\hline \multirow{2}{*}{$20239-4225$} & $\mathrm{BU} 763 \mathrm{AB}$ & HIP 100591 & $\begin{array}{l}13.7362 \\
12.9229\end{array}$ & $\mathrm{y}$ & ${ }_{2}^{2}$ & $\begin{array}{l}104.2 \\
341.8\end{array}$ & $\begin{array}{l}0.3 \\
0.2\end{array}$ & $\begin{array}{l}0.1572 \\
0.3082\end{array}$ & $\begin{array}{l}0.1 \\
0.0\end{array}$ & 0.6 & & -0.047 & (Seymour et al. 2002) \\
\hline & BU 763AB & HIP 100591 & $\begin{array}{l}12.9229 \\
13.7362\end{array}$ & $\begin{array}{l}\mathrm{y} \\
\mathrm{y}\end{array}$ & $\begin{array}{l}2 \\
2\end{array}$ & $\begin{array}{l}341.8 \\
344.5\end{array}$ & $\begin{array}{l}0.2 \\
0.0\end{array}$ & $\begin{array}{l}0.3082 \\
0.3264\end{array}$ & $\begin{array}{l}0.0 \\
0.0\end{array}$ & $\begin{array}{l}1.1 \\
1.1\end{array}$ & $\begin{array}{l}2.7 \\
3.2\end{array}$ & $\begin{array}{l}-0.047 \\
-0.038\end{array}$ & (Seymour et al. 2002 ) \\
\hline \multirow{2}{*}{$20248-1943$} & HDS2919 & HIP 100685 & 12.9229 & I & 2 & $\begin{array}{l}344.0 \\
106.5\end{array}$ & 0.3 & 0.2403 & 0.9 & 0.8 & & & \\
\hline & & & 13.7363 & $\mathrm{I}$ & 2 & 111.3 & 0. & 0.2132 & 0.1 & 0.7 & & & \\
\hline $20269-$ & R 321 & HIP 100852 & 13.7362 & $\mathrm{y}$ & 2 & 126.0 & 0.1 & 1.5667 & 2.1 & $1.9 *$ & 1.3 & 0.001 & $([$ Hein \\
\hline $20289-1749$ & SHJ 3 & HIP 101027 & 13.4740 & $\mathrm{y}$ & 2 & 191.4 & 0.3 & 1.5885 & 0.3 & $1.9 *$ & 2.3 & -0.126 & (Heintz 1986b) \\
\hline $20311-1503$ & FIN 336 & HIP 101221 & 13.47 & $\mathrm{y}$ & 2 & 133.2 & 0.6 & 0.1480 & 1.7 & $1.1:$ & -1.5 & 0.002 & (Mason et al. 2010) \\
\hline \multirow{3}{*}{$20401-2852$} & TOK 369 & HIP 101726 & 13.7362 & I & 2 & 230.1 & 1.4 & 0.4943 & 1.4 & 4.3 & & & \\
\hline & SEE $423 \mathrm{AB}$ & HD 196718 & $\begin{array}{r}12.9229 \\
13.7262\end{array}$ & I & 3 & 39.0 & 6.0 & 1.1747 & 5.2 & 1.4 & & & \\
\hline & SEE $423 B C$ & HD 196718 & $\begin{array}{l}13.7362 \\
12.9229\end{array}$ & $\begin{array}{l}1 \\
\text { I }\end{array}$ & ${ }_{3}^{2}$ & $\begin{array}{r}39.5 \\
282.3\end{array}$ & $\begin{array}{l}1.9 \\
7.9\end{array}$ & $\begin{array}{l}1.1782 \\
0.2041\end{array}$ & $\begin{array}{l}4.9 \\
8.1\end{array}$ & $\begin{array}{l}1.5 \\
0.3\end{array}$ & & & \\
\hline $20401-2852$ & & & 13 & I & 2 & 283.1 & 8.4 & 0.2057 & 0.3 & 0. & & & \\
\hline $\begin{array}{l}20406-2639 \\
20462-2145\end{array}$ & $\begin{array}{l}\text { CHR } 186 \\
\end{array}$ & HIP 102032 & $\begin{array}{l}13.7362 \\
13.7363\end{array}$ & $\mathrm{y}$ & 2 & 131.1 & 0.1 & 0.0350 & 0.7 & 0.8 & & & \\
\hline 2046 & $\begin{array}{l}\text { HDS } 2957 \\
\text { MCA } 64 \mathrm{Aa}, \mathrm{Ab}\end{array}$ & $\begin{array}{l}\text { HIP } 102486 \\
\text { HIP } 103045\end{array}$ & $\begin{array}{l}13.7363 \\
13.7363\end{array}$ & 1 & $\frac{2}{2}$ & $\begin{array}{l}146.2 \\
14.1\end{array}-10$ & $\begin{array}{l}0.2 \\
0.0\end{array}$ & $\begin{array}{l}0.3651 \\
0.0377\end{array}$ & $\begin{array}{l}0.2 \\
0.0\end{array}$ & ${ }_{0}^{2}$. & & & \\
\hline \multirow{3}{*}{$\begin{array}{l}20562-3146 \\
21073-5702\end{array}$} & & & 12.9229 & I & 2 & 215.3 & 0.5 & 0.2659 & 0.5 & 0.7 & 79.0 & -0.027 & (Seymour et al. 2002) \\
\hline & HDS3009 & HIP 104256 & 12.9230 & I & 2 & 143.9 & 0.4 & 0.1995 & 0.4 & 1.6 & & & \\
\hline & & & 12.9230 & $\mathrm{y}$ & 1 & 144.5 & 0.5 & 0.2005 & 0.5 & 2.4 & & & \\
\hline $21074-0814$ & $\mathrm{BU} 368 \mathrm{AB}$ & HIP 104272 & 13.7363 & $\mathrm{y}$ & 2 & 109.7 & 0.1 & 0.0901 & 0.1 & 1.0 & 12.9 & -0.158 & (Olevic \& Cvetkovic 2004b) \\
\hline $21099-2424$ & HDS3015 & HIP 104476 & 12.9230 & I & 2 & 135.1 & 0.1 & 0.0998 & 1.5 & 0.5 & & & \\
\hline & & & $\begin{array}{l}12.9230 \\
13.7363\end{array}$ & $\mathrm{y}$ & $\frac{1}{2}$ & $\begin{array}{l}135.1 \\
155.9\end{array}$ & 0.1 & $\begin{array}{l}0.0986 \\
0.0548\end{array}$ & 0.1 & 0.7 & & & \\
\hline & & & 13.7363 & & & 155.9 & 0.2 & & 0.1 & 1.0 & & & \\
\hline
\end{tabular}


TABLE 2-Continued

\begin{tabular}{|c|c|c|c|c|c|c|c|c|c|c|c|c|c|}
\hline $\begin{array}{l}\text { WDS } \\
(2000)\end{array}$ & $\begin{array}{c}\text { Discoverer } \\
\text { Designation }\end{array}$ & $\begin{array}{l}\text { Other } \\
\text { name }\end{array}$ & $\begin{array}{c}\text { Epoch } \\
+2000 \\
\end{array}$ & Filt & $\mathrm{N}$ & $\begin{array}{c}\theta \\
(\mathrm{deg})\end{array}$ & $\begin{array}{l}\rho \sigma_{\theta} \\
(\mathrm{mas})\end{array}$ & $\begin{array}{l}\rho \\
\left({ }^{\prime \prime}\right) \\
\end{array}$ & $\begin{array}{c}\sigma \rho \\
(\mathrm{mas})\end{array}$ & $\begin{array}{l}\Delta m \\
(\mathrm{mag})\end{array}$ & $\begin{array}{c}{[\mathrm{O}-\mathrm{C}]_{\theta}} \\
(\mathrm{deg})\end{array}$ & $\begin{array}{c}{[\mathrm{O}-\mathrm{C}]_{\rho}} \\
\left({ }^{\prime \prime}\right)\end{array}$ & $\begin{array}{c}\text { Reference } \\
\text { code* }\end{array}$ \\
\hline $21198-2621$ & $\mathrm{BU} 271 \mathrm{AB}$ & HIP 105312 & 12.9230 & $\mathrm{y}$ & 2 & 316.2 & 0.3 & 0.7158 & 0.3 & 3.2 & -0.5 & 0.001 & Table 7 \\
\hline & & & $\begin{array}{l}13.7363 \\
13.7363\end{array}$ & $\mathrm{y}$ & 2 & 321.3 & 0.3 & 0.6799 & 0.6 & $3.2 *$ & -0.5 & 0.012 & \\
\hline $21200-2718$ & BU 252 & HIP 105324 & $\begin{array}{l}13.7363 \\
13.7363\end{array}$ & $\begin{array}{l}\mathrm{y} \\
\mathrm{I}\end{array}$ & $\begin{array}{l}1 \\
1\end{array}$ & $\begin{array}{l}88.6 \\
88.6\end{array}$ & $\begin{array}{l}0.2 \\
0.1\end{array}$ & $\begin{array}{l}2.1271 \\
2.1299\end{array}$ & $\begin{array}{l}0.2 \\
0.1\end{array}$ & $\begin{array}{l}0.1 * \\
0.1 *\end{array}$ & & & \\
\hline $21247-0420$ & HDS3050 & HIP 105715 & 13.7363 & $\mathrm{y}$ & 2 & 304.0 & 0.4 & 0.1473 & 0.4 & 1.6 & & & \\
\hline $21274-0701$ & HDS3053 & HIP 105947 & 13.7363 & $\mathrm{y}$ & 2 & 225.8 & 0.4 & 0.0850 & 1.1 & 1.9 & -19.7 & 0.016 & (Mason et al. 2010) \\
\hline $21310-3633$ & B $1008 \mathrm{AB}$ & HIP 106224 & $\begin{array}{l}12.9230 \\
13.7363\end{array}$ & $\begin{array}{l}\mathrm{y} \\
\mathrm{y}\end{array}$ & $\begin{array}{l}2 \\
2\end{array}$ & $\begin{array}{l}14.6 \\
10.2\end{array}$ & $\begin{array}{l}0.1 \\
0.2\end{array}$ & $\begin{array}{l}0.0985 \\
0.0796\end{array}$ & $\begin{array}{l}0.3 \\
0.5\end{array}$ & $\begin{array}{l}0.5 \\
1.5\end{array}$ & & & \\
\hline $21368-3043$ & VOU $35 \mathrm{AB}$ & HIP 106701 & $\begin{array}{l}12.9230 \\
13.7363\end{array}$ & $\mathrm{y}$ & 2 & 144.0 & 0.1 & 0.1469 & 0.1 & 0.5 & -0.6 & 0.003 & Table 6 \\
\hline $21368-3043$ & TOK $386 \mathrm{AC}$ & HIP 106701 & $\begin{array}{l}13.7363 \\
13.7363\end{array}$ & $\begin{array}{l}1 \\
\mathrm{I}\end{array}$ & $\begin{array}{l}3 \\
3 \\
3\end{array} \longrightarrow x$ & $\begin{array}{r}140.3 \\
53.5\end{array}$ & $\begin{array}{l}1.4 \\
8.9\end{array}$ & $\begin{array}{l}0.1562 \\
0.4506\end{array}$ & $\begin{array}{c}4.7 \\
11.4\end{array}$ & $\begin{array}{l}0.9 \\
4.1\end{array}$ & 0.6 & -0.001 & \\
\hline $21477-3054$ & FIN $330 \mathrm{AB}$ & HIP 107608 & 12.9230 & $\mathrm{y}$ & 2 & 33.0 & 0.2 & 0.1522 & 0.1 & $\begin{array}{l}4.1 \\
0.3\end{array}$ & 0.3 & -0.004 & (Docobo \& Andrade 2013) \\
\hline $21504-5818$ & HDS3109 & HIP 107806 & $\begin{array}{l}12.9230 \\
12.9230\end{array}$ & $\begin{array}{l}\mathrm{y} \\
\mathrm{V}\end{array}$ & $\begin{array}{l}2 \\
2\end{array}$ & $\begin{array}{l}139.2 \\
131.8\end{array}$ & $\begin{array}{l}0.5 \\
0.3\end{array}$ & $\begin{array}{l}0.0372 \\
0.0371\end{array}$ & $\begin{array}{l}0.3 \\
1.0\end{array}$ & $\begin{array}{l}0.3 \\
0.0\end{array}$ & & & \\
\hline $\begin{array}{l}21579-5500 \\
22006-140\end{array}$ & $\begin{array}{l}\text { FIN } 307 \\
\text { TOK } 215\end{array}$ & $\begin{array}{l}\text { HIP } 108431 \\
\text { HIP } 108649\end{array}$ & $\begin{array}{r}12.9230 \\
3764\end{array}$ & $\mathrm{~V}$ & 2 & $\begin{array}{r}289.5 \\
366\end{array}$ & 0.2 & 0.1268 & 0.1 & 0.6 & 5.3 & -0.005 & (Docobo \& Andrade 2013) \\
\hline $22061-0521$ & TOK 373 & HIP 109110 & 13.7364 & I & 2 & $\begin{array}{l}36.6 \\
45.4\end{array}$ & $\begin{array}{l}6.1 \\
0.3\end{array}$ & 0.1129 & $\begin{array}{l}7.1 \\
1.8\end{array}$ & $\begin{array}{l}4.4 \\
2.5\end{array}$ & & & \\
\hline & & & 13.7364 & $\mathrm{y}$ & 2 & 45.7 & 1.1 & 0.1165 & 2.6 & 3.7 & & & \\
\hline $22116-3428$ & $\mathrm{CHR} 230 \mathrm{Aa}, \mathrm{Ab}$ & HIP 109561 & 13.7364 & $\mathrm{y}$ & 1 & 229.6 & 0.0 & 0.0268 & 0.0 & 2.3 & & & \\
\hline $22116-3428$ & $\mathrm{BU} 769 \mathrm{Aa}, \mathrm{B}$ & HIP 109561 & $\begin{array}{l}12.9230 \\
13.7364\end{array}$ & $\mathrm{y}$ & $\begin{array}{l}2 \\
1\end{array}$ & $\begin{array}{l}355.6 \\
355.8\end{array}$ & $\begin{array}{l}0.5 \\
0.0\end{array}$ & $\begin{array}{l}0.8591 \\
0.8585\end{array}$ & $\begin{array}{l}0.1 \\
0.0\end{array}$ & $\begin{array}{l}1.0 * \\
1.2\end{array}$ & & & \\
\hline $22134-3729$ & B 2056 & HD 210767 & 13.7364 & $\begin{array}{l}\mathrm{y} \\
\mathrm{y}\end{array}$ & $\frac{1}{2}$ & $\begin{array}{l}353.8 \\
174.4\end{array}$ & 0.1 & $\begin{array}{l}.8585 \\
0.1778\end{array}$ & 0.1 & 1.6 & 2.0 & 0.006 & (Hartkopf \& Harshaw 2013b) \\
\hline $22152-0535$ & A $2599 \mathrm{AB}$ & HIP 109874 & 13.7364 & $\mathrm{y}$ & 2 & 280.3 & 0.2 & 0.6992 & 0.2 & 2.3 & & & \\
\hline $22156-4121$ & CHR 187 & HIP 109908 & 13.7364 & $\mathrm{y}$ & 2 & 102.0 & 0.1 & 0.1194 & 0.1 & 2.0 & & & \\
\hline $\begin{array}{l}22181-0014 \\
22183-5338\end{array}$ & $\begin{array}{l}\text { CHR } 107 \\
\text { HDO } 298\end{array}$ & $\begin{array}{l}\text { HIP } 110091 \\
\text { HIP } 110109\end{array}$ & 13.7364 & $\mathrm{y}$ & 2 & 261.7 & 0.5 & 0.0216 & 1.2 & $1.3:$ & & & \\
\hline $\begin{array}{l}22183-5338 \\
22228-2937\end{array}$ & $\begin{array}{l}\text { HDO } 298 \\
\text { HDS } 3172\end{array}$ & $\begin{array}{l}\text { HIP } 110109 \\
\text { HIP } 110483\end{array}$ & $\begin{array}{l}12.9230 \\
12.9231\end{array}$ & ${ }_{\mathrm{I}}^{\mathrm{I}}$ & $\begin{array}{r}2 \\
2\end{array}$ & $\begin{array}{r}47.2 \\
337.1\end{array}$ & $\begin{array}{l}1.1 \\
0.2\end{array}$ & $\begin{array}{l}1.9668 \\
0.1727\end{array}$ & $\begin{array}{l}0.6 \\
0.3\end{array}$ & $3.6{ }^{*}$ & & & \\
\hline $22228-2937$ & HDS3172 & HIP 110483 & $\begin{array}{l}12.9231 \\
12.9231\end{array}$ & $\mathrm{~V}$ & $\frac{2}{2}$ & $\begin{array}{l}337.1 \\
336.8\end{array}$ & $\begin{array}{l}0.2 \\
0.8\end{array}$ & $\begin{array}{l}0.1727 \\
0.1734\end{array}-1$ & $\begin{array}{l}0.3 \\
0.5\end{array}$ & $\begin{array}{l}1.7 \\
2.6\end{array}$ & & & \\
\hline & & & 13.7364 & $\mathrm{y}$ & 2 & 335.0 & 0.4 & 0.1714 & 0.6 & 2.5 & & & \\
\hline $22240+0612$ & $\mathrm{RAO} 27 \mathrm{Aa}, \mathrm{Ab}$ & & 13.7365 & $\mathrm{y}$ & 2 & 320.1 & 1.1 & 0.0407 & 0.7 & 1.3 & & & \\
\hline $22288-0001$ & $\mathrm{EBE} 1 \mathrm{Aa}, \mathrm{Ab}$ & HIP 110960 & $\begin{array}{l}12.9229 \\
13.7364\end{array}$ & $\begin{array}{l}\mathrm{I} \\
\mathrm{I}\end{array}$ & $\begin{array}{l}2 \\
2\end{array}$ & $\begin{array}{l}200.4 \\
202.2\end{array}$ & $\begin{array}{l}5.3 \\
0.5\end{array}$ & $\begin{array}{l}0.5838 \\
0.6213\end{array}$ & $\begin{array}{c}11.7 \\
0.2\end{array}$ & $\begin{array}{l}5.4 \\
5.5\end{array}$ & & & \\
\hline $22288-0001$ & STF2909Aa,B & HIP 110960 & 12.9229 & I & 2 & 166.2 & 2.8 & 2.2485 & 12.1 & $1.1 *$ & -1.3 & 0.048 & (Scardia et al. 2010) \\
\hline & & & $\begin{array}{l}13.7364 \\
13.7364\end{array}$ & $\begin{array}{l}\mathrm{y} \\
\mathrm{I}\end{array}$ & $\begin{array}{l}1 \\
2\end{array}$ & $\begin{array}{l}165.7 \\
165.7\end{array}$ & $\begin{array}{l}0.0 \\
0.2\end{array}$ & $\begin{array}{l}2.2710 \\
2.2802\end{array}$ & $\begin{array}{l}0.0 \\
1.9\end{array}$ & $\begin{array}{l}0.2^{*} \\
1.3\end{array}$ & $\begin{array}{l}-0.7 \\
-0.7\end{array}$ & $\begin{array}{l}0.053 \\
0.062\end{array}-1$ & \\
\hline $22443-6030$ & HDS3227 & HIP 112259 & 12.9230 & $\mathrm{y}$ & 3 & 255.3 & 0.3 & 0.0266 & 2.1 & 0.6 & & & \\
\hline & & & 12.9230 & $\mathrm{~V}$ & 1 & 254.7 & 0.0 & 0.0267 & 0.0 & 0.6 & & & \\
\hline $22475-6928$ & HDS3236 & HIP 112525 & 13.7366 & $\mathrm{y}$ & 2 & 2 & 0.1 & & & 0.8 & & & \\
\hline $22508-6543$ & HDS3246 & HIP 112816 & $\begin{array}{l}12.9230 \\
12.9230\end{array}$ & $\stackrel{\mathrm{y}}{\mathrm{y}}$ & $\begin{array}{l}1 \\
2\end{array}$ & $\begin{array}{l}270.3 \\
270.6\end{array}$ & $\begin{array}{l}0.9 \\
0.2\end{array}$ & $\begin{array}{l}0.1611 \\
0.1636\end{array}$ & $\begin{array}{l}0.9 \\
0.6\end{array}$ & $\begin{array}{l}3.3 \\
2.3\end{array}$ & & & \\
\hline & & & 13.7366 & $\mathrm{y}$ & 1 & 268.6 & 1.0 & 0.1079 & 1.0 & 3.5 & & & \\
\hline & & & 13.7 & I & 2 & 269.0 & 0.8 & 0.1099 & 0.6 & 2.3 & & & \\
\hline $22519-4647$ & $\begin{array}{ll}\text { I } 683 \\
\text { fDs }\end{array}$ & HIP 112908 & 12.9230 & I & 2 & 350.8 & 0.5 & 1.9533 & 0.1 & $0.2 *$ & & & \\
\hline $\begin{array}{l}22532-3750 \\
22532-3750\end{array}$ & $\begin{array}{l}\text { HDS3250Aa, Ab } \\
\text { B 2504Aa B }\end{array}$ & $\begin{array}{l}\text { HIP } 113010 \\
\text { HIP } 113010\end{array}$ & $\begin{array}{l}12.9231 \\
12.9231\end{array}$ & $\begin{array}{l}\text { I } \\
\text { I }\end{array}$ & $\begin{array}{r}2 \\
2\end{array}$ & $\begin{array}{r}37.8 \\
278.0\end{array}$ & $\begin{array}{l}5.5 \\
12.2\end{array}$ & $\begin{array}{l}0.1131 \\
2.7196\end{array}$ & 4.3 & 3.1 & & & \\
\hline $22553-4828$ & I $22 \mathrm{AB}$ & HIP 113191 & 12.9230 & $\begin{array}{l}1 \\
\mathrm{y}\end{array}$ & $\frac{2}{2}$ & $\begin{array}{l}278.0 \\
176.0\end{array}$ & $\begin{array}{l}12.2 \\
0.1\end{array}$ & $\begin{array}{l}2.7196 \\
0.5234\end{array}$ & $\begin{array}{l}4.3 \\
0.2\end{array}$ & $\begin{array}{l}3.7 \\
0.5\end{array}$ & & & \\
\hline 238 & HDS3293Aa, Ab & HIP 114119 & 13 & $\mathrm{y}$ & 2 & 34 & 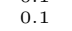 & 0.2 & 0. & 2.2 & & & \\
\hline $23099-2227$ & RST3320 & HIP 114375 & 13.7365 & & 2 & 112.8 & 0.0 & 0.2118 & 0. & & -0.1 & -0.005 & (Hartkopf \& Mason 2010) \\
\hline $23100-4252$ & DON1042 & HIP 114382 & 12.9231 & I & 2 & 301.4 & 0.4 & 0.6807 & 0.2 & $2.7 *$ & 180.4 & 0.004 & Table 6 \\
\hline & & & 12.9231 & $\mathrm{y}$ & 2 & 30 & 0. & 0.6813 & 0.2 & $3.1 *$ & 180.4 & 0.005 & \\
\hline & & & 13.7 & $\mathrm{y}$ & 2 & 30 & 0 & 0.6 & 0.4 & 3.2 & 180.9 & 0.007 & \\
\hline $23171-1349$ & $\mathrm{BU} 182 \mathrm{AB}$ & HIP 114962 & 13.7365 & $\mathrm{y}$ & 2 & 227.6 & 0.1 & 0.7690 & 0. & 1.1 & 1.8 & 0.281 & (Heintz 1991) \\
\hline 2302 & $\begin{array}{l}\text { HDS } 3361 \\
\text { TOK } 375\end{array}$ & $\begin{array}{l}\text { HIP } 116721 \\
\text { HIP } 117247\end{array}$ & $\begin{array}{l}13.7365 \\
13.7365\end{array}$ & $\begin{array}{l}1 \\
\mathrm{I}\end{array}$ & $\begin{array}{r}2 \\
2\end{array}$ & 268.1 & $\begin{array}{l}0.4 \\
0.9\end{array}$ & 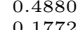 & $\begin{array}{l}0.5 \\
1.9\end{array}$ & 2.8 & & & \\
\hline $\begin{array}{l}23464-2302 \\
23466-0900\end{array}$ & TOK 220 & HIP 117258 & 13.7365 & $\begin{array}{l}1 \\
\mathrm{I}\end{array}$ & $\begin{array}{l}2 \\
2\end{array}$ & $\begin{array}{r}295.8 \\
21.0\end{array}$ & $\begin{array}{l}0.9 \\
0.5\end{array}$ & 0.2099 & $\begin{array}{l}1.9 \\
0.5\end{array}$ & $\begin{array}{l}3.7 \\
3.3\end{array}$ & & & \\
\hline $23474-7118$ & FIN $375 \mathrm{Aa}, \mathrm{Ab}$ & HIP 117326 & 12.9231 & $\mathrm{y}$ & 2 & 244.0 & 0.8 & 0.1215 & 0.6 & 2.4 & 0.0 & 0.001 & Table 7 \\
\hline 23568 > $>20$ & & & $\begin{array}{l}13.7366 \\
13.7365\end{array}$ & $\mathrm{y}$ & ${ }^{2}$ & $\begin{array}{l}248.2 \\
259.5\end{array}$ & $\begin{array}{l}0.2 \\
0.2\end{array}$ & 0.1203 & 0.7 & 2.4 & 0.9 & -0.000 & \\
\hline $\begin{array}{l}23568+0444 \\
23586-1408\end{array}$ & $\begin{array}{l}\text { A } 2100 \\
\text { RST } 4136 \mathrm{AB}\end{array}$ & HIP 118205 & $\begin{array}{l}13.7365 \\
13.7365\end{array}$ & $\begin{array}{l}\mathrm{y} \\
\mathrm{y}\end{array}$ & $\begin{array}{l}2 \\
2\end{array}$ & $\begin{array}{l}259.5 \\
200.8\end{array}$ & $\begin{array}{l}0.2 \\
0.1\end{array}$ & $\begin{array}{l}0.3888 \\
0.1900\end{array}$ & $\begin{array}{l}0.1 \\
0.1\end{array}$ & $\begin{array}{l}1.2 \\
1.0\end{array}$ & $\begin{array}{l}0.3 \\
0.2\end{array}$ & $\begin{array}{r}0.006 \\
-0.009\end{array}$ & $\begin{array}{l}\text { Mason \& Hartopo } 2012) \\
\text { (Docobo \& Campo 2011) }\end{array}$ \\
\hline
\end{tabular}


TABLE 3

UNRESOLVED STARS

\begin{tabular}{|c|c|c|c|c|c|c|c|}
\hline \multirow{2}{*}{ WDS (2000) } & \multirow{2}{*}{$\begin{array}{c}\text { Discoverer } \\
\text { Designation } \\
\text { or other name }\end{array}$} & & Epoch & Filter & $\mathrm{N}$ & $5 \sigma$ Detec & n Limit \\
\hline & & $\begin{array}{l}\text { or other } \\
\text { name }\end{array}$ & +2000 & & & $\begin{array}{c}\Delta m\left(0^{\prime \prime} 15\right) \\
(\mathrm{mag})\end{array}$ & $\begin{array}{c}\Delta m\left(1^{\prime \prime}\right) \\
(\mathrm{mag})\end{array}$ \\
\hline $00219-2300$ & RST5493BC & HIP 1732 & 12.9231 & $\mathrm{y}$ & 2 & 4.91 & 6.54 \\
\hline $00490+1656$ & HIP 3810 & HIP 3810 & 13.7367 & I & 1 & 4.22 & 5.57 \\
\hline & & & 13.7367 & $\mathrm{y}$ & 2 & 4.94 & 6.26 \\
\hline $01144-0755$ & WSI $70 \mathrm{Aa}, \mathrm{Ab}$ & HIP 5799 & 12.9232 & I & 1 & 3.88 & 6.12 \\
\hline $01496-4646$ & TOK 184 & HIP 8498 & 13.7368 & $\mathrm{y}$ & 1 & 4.71 & 5.25 \\
\hline $02128-0224$ & TOK $39 \mathrm{Aa}, \mathrm{Ab}$ & HIP 10305 & 12.9205 & $\mathrm{y}$ & 2 & 4.47 & 6.88 \\
\hline $02136-0849$ & TOK 361 & HIP 10365 & 13.7367 & $\mathrm{y}$ & 1 & 3.88 & 4.71 \\
\hline & & & 13.7367 & I & 2 & $\begin{array}{l}3.12 \\
3.12\end{array}$ & 4.59 \\
\hline $02321-1515$ & TOK $382 \mathrm{Aa}, \mathrm{Ab}$ & HIP 11783 & 13.7478 & $\mathrm{y}$ & 1 & 3.92 & 4.47 \\
\hline $02449+1007$ & $\mathrm{TOK} 1 \mathrm{Aa}, \mathrm{Ab}$ & HIP 12828 & 12.9232 & $\mathrm{y}$ & 2 & 5.00 & 6.60 \\
\hline $02568-3609$ & $\mathrm{HDS} 378 \mathrm{Aa}, \mathrm{Ab}$ & HIP 13725 & 12.9233 & I & 2 & 3.52 & 4.89 \\
\hline $03099-0654$ & TOK 188 & HIP 14699 & 12.9205 & $\mathrm{~V}$ & 1 & 2.83 & 3.61 \\
\hline $03182-6230$ & $\mathrm{BNU} 2 \mathrm{Aa}, \mathrm{Ab}$ & HIP 15371 & 12.9232 & $\mathrm{y}$ & 2 & 4.72 & 6.88 \\
\hline $03244-1539$ & A $2909 \mathrm{AB}$ & HIP 15868 & 12.9233 & $\mathrm{y}$ & 2 & 4.61 & 5.94 \\
\hline $03309-6200$ & TOK 190 & HIP 16370 & 12.9232 & $\mathrm{y}$ & 1 & 4.48 & 6.16 \\
\hline $03442-6448$ & TOK $191 \mathrm{Aa}, \mathrm{Ab}$ & HIP 17440 & 12.9232 & $\mathrm{y}$ & 2 & 4.80 & 7.32 \\
\hline & & & 13.7368 & $\mathrm{y}$ & 2 & 5.44 & 6.79 \\
\hline $04044-1014$ & HIP 19016 & HIP 19016 & 13.7369 & I & 2 & 4.50 & 6.24 \\
\hline $04053+2201$ & $\mathrm{CHR} 158 \mathrm{Aa}, \mathrm{Ab}$ & HIP 19076 & 13.7369 & $\mathrm{y}$ & 2 & 5.00 & 6.57 \\
\hline $04345+1001$ & HIP 21308 & HIP 21308 & 13.1310 & I & 2 & 3.23 & 4.22 \\
\hline $04583+0027$ & HIP 23105 & HIP 23105 & 13.1310 & $\mathrm{y}$ & 2 & 4.54 & 5.32 \\
\hline $05017+2640$ & $\mathrm{BAG} 21 \mathrm{Ca}, \mathrm{Cb}$ & HIP 23402 & 13.1310 & $\mathrm{y}$ & 1 & 3.35 & 3.63 \\
\hline $05066-7734$ & HIP 23776 & HIP 23776 & 13.1283 & $\mathrm{y}$ & 2 & 4.03 & 5.03 \\
\hline $05074+1839$ & A 3010 & HIP 23835 & 12.9206 & $\mathrm{y}$ & 2 & 4.89 & 6.95 \\
\hline & & & 13.1310 & $\mathrm{y}$ & 2 & 4.97 & 6.51 \\
\hline & & & 13.7370 & $\mathrm{y}$ & 2 & 5.21 & 6.68 \\
\hline $05229-4219$ & TOK 93Aa, $\mathrm{Ab}$ & HIP 25148 & 12.8335 & $\mathrm{y}$ & 2 & 3.76 & 4.47 \\
\hline $00223-4213$ & 1 С & 1111200140 & 13.1283 & I & 2 & 3.35 & 4.45 \\
\hline & & & 13.1283 & $\mathrm{~V}$ & 1 & 3.67 & 4.18 \\
\hline & & & 13.7369 & I & 2 & 4.44 & 5.72 \\
\hline & & & 13.7369 & $\mathrm{y}$ & 2 & 4.45 & 5.03 \\
\hline $05271-1154$ & HIP 25486 & HIP 25486 & 13.1311 & $\mathrm{y}$ & 2 & 4.73 & 6.95 \\
\hline $05402+1211$ & $\operatorname{RAS} 1$ & HIP 26676 & 12.9206 & I & 3 & 2.66 & 3.48 \\
\hline $05498-7225$ & TOK 363 & HIP 27531 & 13.1283 & I & 2 & 2.63 & 3.02 \\
\hline $06033-1603$ & HIP 28678 & HIP 28678 & 12.9207 & $\mathrm{y}$ & 2 & 4.48 & 5.72 \\
\hline & & & 12.9207 & I & 2 & 2.99 & 5.20 \\
\hline $06048-4828$ & DUN 23 & HIP 28796 & 13.1312 & $\mathrm{y}$ & 2 & 4.66 & 5.54 \\
\hline $06127-4338$ & HIP 29479 & HIP 29479 & 13.7370 & I & 2 & 4.28 & 5.97 \\
\hline $06290+2013$ & $\mathrm{BTZ} 1 \mathrm{Aa}, \mathrm{Ab}$ & HIP 30883 & 12.9207 & I & 2 & 3.48 & 4.56 \\
\hline & & & 12.9207 & $\mathrm{y}$ & 1 & 4.61 & 6.05 \\
\hline & & & 13.1310 & $\mathrm{y}$ & 2 & 4.86 & 5.97 \\
\hline & & & 13.1310 & I & 1 & 3.14 & 4.59 \\
\hline $06336-3939$ & HIP 31280 & HIP 31280 & 13.1312 & $\mathrm{y}$ & 2 & 4.66 & 5.66 \\
\hline & & & 13.1312 & I & 1 & 3.85 & 5.84 \\
\hline $06371-3214$ & HIP 31623 & HIP 31623 & 13.1312 & $\mathrm{y}$ & 2 & 4.73 & 6.04 \\
\hline $07345-5258$ & HIP36832 & HIP 36832 & 12.8335 & $\mathrm{y}$ & 2 & 4.43 & 5.26 \\
\hline & & & 12.8335 & I & 2 & 3.43 & 5.39 \\
\hline $07383-2522$ & B 731 & HIP 37173 & 12.9208 & $\mathrm{y}$ & 2 & 4.84 & 7.42 \\
\hline & & & 13.1313 & $\mathrm{y}$ & 2 & 5.29 & 7.57 \\
\hline $07387-3144$ & HIP 37224 & HIP 37224 & 13.1313 & $\mathrm{y}$ & 1 & 4.69 & 5.55 \\
\hline & & & 13.1313 & I & 2 & 4.28 & 6.08 \\
\hline $07430-4511$ & WSI 99 & HIP 37606 & 12.8335 & $\mathrm{y}$ & 2 & 5.23 & 6.93 \\
\hline & & & 13.1312 & $\mathrm{y}$ & 2 & 4.82 & 6.69 \\
\hline $07522-4035$ & TOK 195 & HIP 38414 & 13.1313 & $\mathrm{y}$ & 2 & 5.13 & 7.30 \\
\hline $08095-4720$ & TOK $2 \mathrm{Aa}, \mathrm{Ab}$ & HIP 39953 & 12.9209 & $\mathrm{y}$ & 2 & 5.16 & 7.50 \\
\hline & & & 13.1287 & $\mathrm{y}$ & 2 & 4.59 & 7.13 \\
\hline $08151-0655$ & MET 52 & HIP 40419 & 12.9209 & $\mathrm{y}$ & 2 & 4.05 & 4.94 \\
\hline $08228-2621$ & B $767 \mathrm{AB}$ & HIP 41074 & 12.9209 & $\mathrm{y}$ & 2 & 5.07 & 6.90 \\
\hline & & & 12.9209 & I & 1 & 3.91 & 6.27 \\
\hline & & & 13.1314 & $\mathrm{y}$ & 2 & 5.14 & 7.37 \\
\hline $08246-0109$ & B $2527 \mathrm{AB}$ & HIP 41209 & 12.9209 & $\mathrm{y}$ & 2 & 4.61 & 5.77 \\
\hline & & & 12.9209 & I & 1 & 3.40 & 5.58 \\
\hline & & & 13.1285 & I & 2 & 4.15 & 4.92 \\
\hline $08246-0345$ & $\mathrm{CHR} 172 \mathrm{Aa}, \mathrm{Ab}$ & HIP 41211 & 12.9209 & $\mathrm{y}$ & 2 & 4.80 & 6.52 \\
\hline & & & 13.1285 & $\mathrm{y}$ & 2 & 4.75 & 6.39 \\
\hline $08326-1502$ & B 2528 & HIP 41893 & 12.9209 & $\mathrm{y}$ & 2 & 4.83 & 6.46 \\
\hline & & & 13.1314 & $\mathrm{y}$ & 2 & 4.63 & 6.60 \\
\hline $08388-0557$ & HIP 42408 & HIP 42408 & 13.1285 & $\mathrm{y}$ & 1 & 3.96 & 4.48 \\
\hline & & & 13.1285 & I & 2 & 3.67 & 4.81 \\
\hline & & & 13.1285 & $\mathrm{R}$ & 2 & 4.15 & 5.17 \\
\hline $08397+0546$ & HIP 42488B & HIP 42491 & 13.1286 & I & 1 & 3.38 & 4.28 \\
\hline & & & 13.1286 & $\mathrm{~V}$ & 2 & 4.04 & 4.73 \\
\hline $08398-6604$ & HDS 1245 & HIP 42496 & 12.9208 & I & 2 & 2.59 & 3.53 \\
\hline & & & 12.9208 & $\mathrm{y}$ & 1 & 3.08 & 3.58 \\
\hline $09125-4337$ & FIN $317 \mathrm{Aa}, \mathrm{Ab}$ & HIP 45189 & 13.1315 & $\mathrm{y}$ & 2 & 5.08 & 7.00 \\
\hline $09191-4128$ & CHR 239 & HIP 45705 & 13.0796 & $\mathrm{y}$ & 2 & 3.84 & 5.04 \\
\hline $09276-3500$ & B 2215 & HIP 46396 & 13.0796 & $\mathrm{y}$ & 2 & 4.45 & 6.07 \\
\hline $09306-4736$ & HIP 46631 & HIP 46631 & 13.2380 & I & 3 & 4.27 & 5.86 \\
\hline $09380-5924$ & HIP 47263 & HIP 47263 & 13.2380 & $\mathrm{y}$ & 2 & 4.73 & 5.47 \\
\hline & & & 13.2380 & I & 2 & 4.15 & 5.58 \\
\hline $09416-3830$ & TOK 198 & HIP 47543 & 13.0796 & $\mathrm{y}$ & 2 & 3.95 & 4.79 \\
\hline & & & 13.1315 & $\mathrm{y}$ & 1 & 4.80 & 5.92 \\
\hline $09518-5053$ & DX Vel & DX Vel & 13.1287 & I & 2 & 2.39 & 2.98 \\
\hline $10066-1919$ & HIP 49520 & HIP 49520 & 13.1289 & I & 2 & 3.89 & 6.04 \\
\hline
\end{tabular}


TABle 3-Continued

\begin{tabular}{|c|c|c|c|c|c|c|c|}
\hline \multirow[t]{2}{*}{ WDS (2000) } & \multirow{2}{*}{$\begin{array}{c}\text { Discoverer } \\
\text { Designation } \\
\text { or other name }\end{array}$} & & Epoch & Filter & $\mathrm{N}$ & $5 \sigma$ Detec & n Limit \\
\hline & & $\begin{array}{l}\text { or other } \\
\text { name }\end{array}$ & +2000 & & & $\begin{array}{c}\Delta m\left(0^{\prime \prime} 15\right) \\
(\mathrm{mag})\end{array}$ & $\begin{array}{c}\Delta m\left(1^{\prime \prime}\right) \\
(\mathrm{mag})\end{array}$ \\
\hline $10232+0542$ & TOK $200 \mathrm{Aa}, \mathrm{Ab}$ & HIP 50870 & 13.1316 & I & 2 & 1.60 & 3.84 \\
\hline & & & 13.1316 & $\mathrm{y}$ & 2 & 4.26 & 5.76 \\
\hline $11014-1204$ & HDS 1572 & HIP 53879 & 13.2381 & y & 4 & 4.81 & 5.79 \\
\hline $11056-1105$ & TOK 221 & HIP 54214 & 13.2381 & $\mathrm{y}$ & 1 & 4.82 & 6.79 \\
\hline $11121-2120$ & HIP 54707 & HIP 54707 & 13.2380 & $\mathrm{y}$ & 1 & 4.70 & 5.78 \\
\hline & & & 13.2380 & I & 2 & 4.09 & 5.83 \\
\hline $11238-3829$ & CHR 241 & HIP 55628 & 13.2380 & $\mathrm{y}$ & 2 & 4.77 & 6.14 \\
\hline & & & 13.2380 & I & 2 & 4.19 & 5.84 \\
\hline $11284+0247$ & HIP 55982 & HIP 55982 & 13.1290 & $\mathrm{y}$ & 1 & 4.59 & 6.11 \\
\hline & & & 13.1290 & I & 2 & 3.16 & 5.33 \\
\hline & & & 13.2381 & I & 2 & 4.12 & 5.65 \\
\hline $11317+1422$ & WSI $101 \mathrm{Aa}, \mathrm{Ab}$ & HIP 56242 & 13.1290 & $\mathrm{y}$ & 2 & 4.19 & 6.39 \\
\hline $11323-0025$ & HIP 56282 & HIP 56282 & 13.2381 & I & 4 & 4.10 & 5.38 \\
\hline & & & 13.2381 & $\mathrm{y}$ & 1 & 4.37 & 4.99 \\
\hline $11414-4101$ & HIP 57021 & HIP 57021 & 13.2380 & y & 2 & 5.09 & 6.50 \\
\hline $11510-0520$ & $\mathrm{MCA} 36$ & HIP 57791 & 13.2381 & $\mathrm{y}$ & 2 & 5.24 & 6.70 \\
\hline & & & 13.2381 & I & 2 & 3.91 & 6.44 \\
\hline $12073-6441$ & HIP 59111 & HIP 59111 & 13.1318 & y & 2 & 4.63 & 6.05 \\
\hline $12532-0333$ & CHR 38 & HIP 62875 & 13.1290 & $\mathrm{y}$ & 2 & 4.78 & 7.13 \\
\hline & & & 13.1290 & I & 1 & 3.74 & 5.72 \\
\hline $13143-5906$ & FIN 205 & HIP 64583 & 13.1291 & I & 2 & 4.88 & 6.01 \\
\hline $13254-5947$ & WSI 76 & HIP 65492 & 13.1291 & $\mathrm{y}$ & 1 & 3.35 & 3.96 \\
\hline $13393-6740$ & HIP 66618 & HIP 66618 & 13.1291 & $\mathrm{y}$ & 2 & 4.43 & 5.48 \\
\hline $13517-5726$ & HIP 67655 & HIP 67655 & 13.1318 & I & 2 & 3.56 & 5.58 \\
\hline $14282-2929$ & FIN $306 \mathrm{AB}$ & HIP 70753 & 13.1318 & $\mathrm{y}$ & 2 & 5.02 & 7.44 \\
\hline $14292-2907$ & HIP 70839 & HIP 70839 & 13.1318 & $\mathrm{y}$ & 1 & 5.00 & 6.99 \\
\hline & & & 13.1318 & I & 1 & 3.96 & 6.08 \\
\hline $14485-3551$ & B $2024 \mathrm{AB}$ & HIP 72427 & 13.1318 & y & 2 & 5.16 & 7.24 \\
\hline & & & 13.1318 & I & 1 & 3.88 & 6.21 \\
\hline $14598-2201$ & TOK 47 & HIP 73385 & 13.1318 & $\mathrm{y}$ & 3 & 4.71 & 6.24 \\
\hline $15006+0836$ & YSC 8 & HIP 73449 & 13.1319 & $\mathrm{y}$ & 2 & 4.20 & 5.35 \\
\hline & & & 13.1319 & I & 1 & 2.83 & 4.53 \\
\hline & & & 13.1319 & y & 2 & 4.20 & 5.35 \\
\hline $15317+0053$ & TOK 48 & HIP 76031 & 13.1319 & $\mathrm{y}$ & 2 & 4.36 & 5.50 \\
\hline $15362-3306$ & FIN 231 & HIP 76395 & 13.1319 & y & 2 & 5.20 & 7.41 \\
\hline $15384-1955$ & CHR 48 & HIP 76582 & 13.1319 & $\mathrm{y}$ & 1 & 5.03 & 6.98 \\
\hline $15453-5841$ & FIN $234 \mathrm{AB}$ & HIP 77160 & 13.1319 & $\mathrm{y}$ & 3 & 4.57 & 5.74 \\
\hline $15467-3441$ & B 847 & HIP 77286 & 13.1319 & $\mathrm{y}$ & 2 & 5.20 & 7.58 \\
\hline $15467-4314$ & I 1276 & HIP 77282 & 13.1319 & I & 2 & 4.37 & 6.09 \\
\hline $17162+0211$ & CHR 61 & HIP 84479 & 13.4736 & $\mathrm{y}$ & 2 & 5.01 & 6.53 \\
\hline $17200-0801$ & CHR $151 \mathrm{Aa}, \mathrm{Ab}$ & HIP 84803 & 13.4737 & $\mathrm{y}$ & 2 & 4.53 & 5.79 \\
\hline $17390+0240$ & WSI 88 & HIP 91438 & 13.7470 & $\mathrm{y}$ & 2 & 5.13 & 7.01 \\
\hline $18174-2817$ & HIP 89620 & HIP 89620 & 13.4738 & I & 2 & 3.25 & 4.22 \\
\hline $18218-1619$ & $\mathrm{CHR} 69$ & HIP 90001 & 13.7470 & $\mathrm{y}$ & 2 & 4.90 & 5.93 \\
\hline $18389-2103$ & WSI 90 & HIP 91438 & 13.4738 & y & 2 & 4.00 & 4.80 \\
\hline & & & 13.4738 & I & 3 & 3.92 & 5.61 \\
\hline $18501-0823$ & HDS2670 & HIP 92423 & 13.7471 & y & 2 & 4.52 & 5.58 \\
\hline $18530-0935$ & CHR 81 & HIP 92674 & 13.4737 & $\mathrm{y}$ & 2 & 4.94 & 6.78 \\
\hline $19098-2101$ & FIN $311 \mathrm{AB}$ & HIP 94141 & 13.4739 & $\mathrm{y}$ & 2 & 4.80 & 6.89 \\
\hline $20224-5630$ & FIN 276 & HIP 100462 & 13.7362 & $\mathrm{y}$ & 1 & 4.15 & 5.02 \\
\hline & & & 13.7362 & I & 3 & 4.15 & 5.66 \\
\hline $20254-2840$ & CHR 97 & HIP 100738 & 13.4740 & y & 2 & 4.90 & 5.86 \\
\hline $20273-1813$ & $\mathrm{CHR} 184 \mathrm{Aa}, \mathrm{Ab}$ & HIP 100881 & 13.4740 & $\mathrm{y}$ & 2 & 4.72 & 6.50 \\
\hline $20441-4446$ & HIP 102338 & HIP 102338 & 13.7362 & I & 2 & 4.15 & 5.67 \\
\hline $20531-1134$ & CHR 101 & HIP 103077 & 13.7363 & y & 1 & 4.95 & 6.85 \\
\hline & & & 13.7363 & I & 2 & 4.00 & 6.42 \\
\hline $20568-2618$ & GJ 811 & HIP 103389 & 13.4740 & I & 2 & 3.82 & 5.39 \\
\hline & & & 13.4740 & y & 3 & 4.75 & 6.47 \\
\hline & & & 13.7362 & $\mathrm{y}$ & 2 & 5.16 & 7.21 \\
\hline & & & 13.7362 & I & 1 & 4.56 & 6.85 \\
\hline $21265-3111$ & TOK 372 & HIP 105872 & 13.7363 & I & 4 & 3.88 & 5.33 \\
\hline $21378-0751$ & $\mathrm{MCA} 68$ & HIP 106786 & 13.7363 & $\mathrm{y}$ & 2 & 5.03 & 6.78 \\
\hline $22006-1410$ & TOK 215 & HIP 108649 & 13.7364 & $\mathrm{y}$ & 1 & 4.94 & 6.02 \\
\hline $22181-0014$ & CHR 107 & HIP 110091 & 12.9229 & $\mathrm{y}$ & 2 & 4.62 & 6.53 \\
\hline $22313-0633$ & CHR 111 & HIP 111170 & 13.7364 & I & 2 & 4.22 & 6.34 \\
\hline & & & 13.7365 & $\mathrm{y}$ & 2 & 5.08 & 6.66 \\
\hline $22443-6030$ & HDS3227 & HIP 112259 & 13.7366 & $\mathrm{y}$ & 2 & 4.99 & 6.19 \\
\hline $22553-4828$ & B $2506 \mathrm{CD}$ & HIP 113190 & 12.9230 & I & 1 & 3.91 & 5.87 \\
\hline & & & 12.9230 & $\mathrm{y}$ & 2 & 4.87 & 6.76 \\
\hline $22583-0224$ & CHR 116 & HIP 113421 & 13.7365 & $\mathrm{y}$ & 2 & 5.12 & 6.60 \\
\hline $23086-6352$ & B 590 & HIP 114269 & 12.9231 & $\mathrm{y}$ & 1 & 4.51 & 6.00 \\
\hline $23114-4259$ & B 594 & HD 218812 & 12.9231 & I & 1 & 3.89 & 5.92 \\
\hline & & & 12.9231 & $\mathrm{y}$ & 2 & 4.54 & 5.53 \\
\hline & & & 13.7366 & $\mathrm{y}$ & 1 & 4.65 & 5.33 \\
\hline & & & 13.7366 & I & 2 & 4.16 & 6.50 \\
\hline $23157+0118$ & CHR 141 & HIP 114834 & 13.7365 & $\mathrm{y}$ & 2 & 4.99 & 6.16 \\
\hline & & & 13.7365 & I & 2 & 4.22 & 6.10 \\
\hline $23162-5947$ & TOK 217 & HIP 114880 & 12.9231 & I & 2 & 3.48 & 5.53 \\
\hline & & & 12.9231 & $\mathrm{y}$ & 1 & 4.43 & 5.50 \\
\hline $23307-2412$ & HIP 116033 & HIP 116033 & 13.7365 & $\mathrm{y}$ & 2 & 5.04 & 6.35 \\
\hline & & & 13.7365 & I & 1 & 4.41 & 6.52 \\
\hline $23318-2115$ & TOK 219 & HIP 116125 & 13.7365 & I & 2 & 4.19 & 6.21 \\
\hline $23438-1517$ & HEG $2 \mathrm{AB}$ & HIP 117054 & 13.7365 & I & 2 & 4.07 & 5.83 \\
\hline $23444-7029$ & WSI 94 & HIP 117105 & 12.9231 & y & 2 & 4.60 & 6.34 \\
\hline
\end{tabular}


TABle 3-Continued

\begin{tabular}{|c|c|c|c|c|c|c|c|}
\hline WDS (2000) & $\begin{array}{c}\text { Discoverer } \\
\text { Designation } \\
\text { or other name } \\
\end{array}$ & $\begin{array}{c}\text { Hipparcos } \\
\text { or other } \\
\text { name }\end{array}$ & $\begin{array}{l}\text { Epoch } \\
+2000\end{array}$ & Filter & $\mathrm{N}$ & $\begin{array}{c}5 \sigma \text { Detec } \\
\Delta m\left(0^{\prime \prime} 15\right) \\
(\mathrm{mag})\end{array}$ & $\begin{array}{c}\text { Limit } \\
\Delta m\left(1^{\prime \prime}\right) \\
(\mathrm{mag})\end{array}$ \\
\hline $23492+0459$ & TOK 376 & HIP 117475 & $\begin{array}{l}13.7366 \\
13.7365 \\
13.7365\end{array}$ & $\begin{array}{l}\mathrm{y} \\
\mathrm{I} \\
\mathrm{y}\end{array}$ & $\begin{array}{l}2 \\
2 \\
1\end{array}$ & $\begin{array}{l}5.07 \\
4.05 \\
4.41\end{array}$ & $\begin{array}{l}6.57 \\
5.02 \\
5.02\end{array}$ \\
\hline
\end{tabular}

TABle 4

NEW PAIRS

\begin{tabular}{clcccrcc}
\hline \hline $\begin{array}{c}\text { WDS } \\
(2000)\end{array}$ & $\begin{array}{c}\text { Discoverer } \\
\text { Designation }\end{array}$ & $\begin{array}{c}\text { Other } \\
\text { name }\end{array}$ & $\begin{array}{c}\text { Epoch } \\
+2000\end{array}$ & Filt & $\begin{array}{c}\theta \\
(\mathrm{deg})\end{array}$ & $\begin{array}{c}\Delta m \\
\left({ }^{\prime \prime}\right)\end{array}$ & $\begin{array}{c}\Delta m \\
(\mathrm{mag})\end{array}$ \\
\hline $02321-1515$ & TOK 382Aa,Ab & HIP 11783 & 13.7478 & $\mathrm{I}$ & 341.8 & 0.2101 & 4.0 \\
$04007+2023$ & TOK 363Aa,Ab & HIP 18719 & 13.7369 & $\mathrm{y}$ & 95.7 & 0.1023 & 5.2 \\
$07435+0329$ & TOK 355Aa,Ab & HIP 37645 & 13.1285 & $\mathrm{y}$ & 330.2 & 0.2078 & 3.7 \\
$08391-5557$ & TOK 356BC & HIP 42424 & 13.1287 & $\mathrm{y}$ & 309.4 & 0.0861 & 2.7 \\
$09086-2550$ & TOK 357BC & HIP 44874 & 13.1314 & $\mathrm{I}$ & 219.2 & 0.1258 & 0.9 \\
$09383+0150$ & TOK 358 & HIP 47292 & 13.1286 & $\mathrm{y}$ & 344.4 & 0.4533 & 3.7 \\
$11192-1950$ & TOK 383Aa,Ab & HD 98412 & 13.2380 & $\mathrm{y}$ & 25.8 & 0.0518 & 0.3 \\
$11420-1701$ & TOK 384Aa,Ab & HIP 57078 & 13.2381 & $\mathrm{y}$ & 128.5 & 0.1467 & 3.6 \\
$11420-1701$ & TOK 384Aa,B & HIP 57078 & 13.2381 & $\mathrm{I}$ & 95.0 & 1.1089 & 5.3 \\
$12509-5743$ & TOK 385 & HIP 62699 & 13.2382 & $\mathrm{I}$ & 112.9 & 0.1868 & 3.6 \\
$21368-3043$ & TOK 386AC & HIP 106701 & 13.7363 & $\mathrm{I}$ & 53.5 & 0.4506 & 4.1 \\
$21368-3043$ & TOK 386AD & HIP 106701 & 00.5622 & $\mathrm{~J}$ & 234.0 & 11.21 & 2.9 \\
\hline
\end{tabular}

TABle 5

LIKELY SPURIOUS BINARIES

\begin{tabular}{clccc}
\hline \hline $\begin{array}{c}\text { WDS } \\
(2000)\end{array}$ & $\begin{array}{c}\text { Discoverer } \\
\text { Designation }\end{array}$ & $\begin{array}{c}\text { Last } \\
\text { measure }\end{array}$ & $\begin{array}{c}\text { SOAR } \\
\text { dates }\end{array}$ & $N_{\text {UR }}$ \\
\hline $03182-6230$ & BNU 2Aa,Ab & 1978 & $2008-2012$ & 3 \\
$07383-2522$ & B 731 & 1966 & $2009-2013$ & 5 \\
$08246-0109$ & B $2527 \mathrm{AB}$ & 1961 & $2010-2013$ & 3 \\
$08246-0345$ & $\mathrm{CHR} 172 \mathrm{Aa}, \mathrm{Ab}$ & 1988 & $2011-2013$ & 3 \\
$08326-1502$ & $\mathrm{~B} 2528$ & 1942 & $2009-2013$ & 4 \\
$23114-4259$ & $\mathrm{~B} 594$ & 1963 & $2008-2012$ & 3 \\
\hline
\end{tabular}


TABLE 6

First Orbital Elements

\begin{tabular}{|c|c|c|c|c|c|c|c|c|c|c|c|c|}
\hline \multirow{2}{*}{\begin{tabular}{l}
\multicolumn{1}{c}{$\begin{array}{l}\text { WDS } \\
\text { (Figure) }\end{array}$} \\
$\begin{array}{l}02022-2402 \\
(3)\end{array}$
\end{tabular}} & \multicolumn{2}{|c|}{$\begin{array}{c}\text { Discoverer } \\
\text { Designation }\end{array}$} & \multirow{2}{*}{$\begin{array}{c}P \\
(\mathrm{yr}) \\
13.88 \\
\pm 4.37\end{array}$} & \multirow{2}{*}{$\begin{array}{c}\begin{array}{l}T_{\circ} \\
(\mathrm{yr})\end{array} \\
\begin{array}{r}2006.3 \\
\pm 3.3\end{array}\end{array}$} & \multirow{2}{*}{$\begin{array}{c}e \\
0.09 \\
\pm 0.15\end{array}$} & \multirow{2}{*}{$\begin{array}{c}\Omega \\
(0) \\
134.4 \\
\pm 17\end{array}$} & \multirow{2}{*}{$\begin{array}{c}a \\
\left(\begin{array}{c}a \\
(\prime)\end{array}\right. \\
0.099 \\
\pm 0.024\end{array}$} & \multirow{2}{*}{$\begin{array}{c}i \\
(\circ) \\
154.2 \\
\pm 23\end{array}$} & \multirow{2}{*}{$\begin{array}{c}\omega \\
(\circ) \\
232.1 \\
\pm 121\end{array}$} & \multirow{2}{*}{$\begin{array}{r}\text { Gr. } \\
3\end{array}$} & \multirow{2}{*}{$\begin{array}{c}\begin{array}{c}\pi_{\mathrm{HIP}} \\
(\mathrm{mas})\end{array} \\
16.1\end{array}$} & \multirow{2}{*}{$\begin{array}{r}\sum \mathcal{M} \\
\mathcal{M}_{\odot} \\
1.2\end{array}$} \\
\hline & TOK & $41 \mathrm{Ba}, \mathrm{Bb}$ & & & & & & & & & & \\
\hline $\begin{array}{l}02572-2458 \\
\text { (4) }\end{array}$ & BEU & $4 \mathrm{Ca}, \mathrm{Cb}$ & $\begin{array}{r}1.519 \\
*\end{array}$ & $\begin{array}{r}2007.103 \\
\pm 0.018\end{array}$ & $\begin{array}{r}0.558 \\
*\end{array}$ & $\begin{array}{r}89.5 \\
\pm 3.1\end{array}$ & $\begin{array}{r}0.060 \\
\pm 0.005\end{array}$ & $\begin{array}{r}179.6 \\
*\end{array}$ & $\begin{array}{r}250.6 \\
*\end{array}$ & 1 & 39.9 & 1.5 \\
\hline $\begin{array}{l}04008+0505 \\
(5)\end{array}$ & A & 1937 & $\begin{array}{r}41.90 \\
\pm 0.57\end{array}$ & $\begin{array}{r}2014.1 \\
\pm 27.0\end{array}$ & $\begin{array}{r}0.699 \\
\pm 0.038\end{array}$ & $\begin{array}{r}185 \\
\pm 23\end{array}$ & $\begin{array}{r}0.096 \\
\pm 0.002\end{array}$ & $\begin{array}{r}41 \\
\pm 143\end{array}$ & $\begin{array}{r}38 \\
\pm 32\end{array}$ & 2 & 5.4 & 3.2 \\
\hline $\begin{array}{l}04215-2055 \\
(6)\end{array}$ & B & $1935 \mathrm{AB}$ & $\begin{array}{r}21.22 \\
\pm 0.19\end{array}$ & $\begin{array}{r}2002.16 \\
\pm 0.22\end{array}$ & $\begin{array}{r}0.505 \\
\pm 0.028\end{array}$ & $\begin{array}{r}239.5 \\
\pm 19.1\end{array}$ & $\begin{array}{r}0.234 \\
\pm 0.006\end{array}$ & $\begin{array}{r}155.2 \\
\pm 4.8\end{array}$ & $\begin{array}{r}310.9 \\
\pm 20.0\end{array}$ & 5 & 25.9 & 1.6 \\
\hline $\begin{array}{l}05072-1924 \\
(7)\end{array}$ & FIN & 376 & $\begin{array}{r}3.889 \\
\pm 0.006\end{array}$ & $\begin{array}{r}2007.269 \\
\pm 0.015\end{array}$ & $\begin{array}{r}0.475 \\
\pm 0.010\end{array}$ & $\begin{array}{r}49.8 \\
\pm 0.5\end{array}$ & $\begin{array}{r}0.085 \\
\pm 0.001\end{array}$ & $\begin{array}{r}91.3 \\
\pm 0.9\end{array}$ & $\begin{array}{r}203.7 \\
\pm 1.4\end{array}$ & 2 & 26.0 & 2.3 \\
\hline $\begin{array}{l}07478-0332 \\
(8)\end{array}$ & RST 4 & 1375 & $\begin{array}{r}80.82 \\
\pm 6.45\end{array}$ & $\begin{array}{r}1998.17 \\
\pm 1.12\end{array}$ & $\begin{array}{r}0.653 \\
\pm 0.026\end{array}$ & $\begin{array}{r}110.5 \\
\pm 4.2\end{array}$ & $\begin{array}{r}0.200 \\
\pm 0.007\end{array}$ & $\begin{array}{r}117.1 \\
\pm 2.4\end{array}$ & $\begin{array}{r}65.3 \\
\pm 3.1\end{array}$ & 5 & 5.7 & 6.5 \\
\hline $\begin{array}{l}09191-4128 \\
(9)\end{array}$ & CHR & 239 & $\begin{array}{r}10.86 \\
\pm 0.02\end{array}$ & $\begin{array}{r}2013.51 \\
\pm 0.02\end{array}$ & $\begin{array}{r}0.394 \\
\pm 0.006\end{array}$ & $\begin{array}{r}152.6 \\
\pm 2.3\end{array}$ & $\begin{array}{r}0.122 \\
\pm 0.001\end{array}$ & $\begin{array}{r}147.0 \\
\pm 1.3\end{array}$ & $\begin{array}{r}260.1 \\
\pm 1.9\end{array}$ & 3 & 20.8 & 1.7 \\
\hline $\begin{array}{l}11514+1148 \\
(10)\end{array}$ & HDS 1 & 672 & $\begin{array}{r}53.79 \\
\pm 11.28\end{array}$ & $\begin{array}{r}2007.07 \\
\pm 0.31\end{array}$ & $\begin{array}{r}0.659 \\
\pm 0.053\end{array}$ & $\begin{array}{r}175.3 \\
\pm 6.9\end{array}$ & $\begin{array}{r}0.361 \\
\pm 0.045\end{array}$ & $\begin{array}{r}47.0 \\
\pm 3.4\end{array}$ & $\begin{array}{r}156.9 \\
\pm 8.0\end{array}$ & 4 & 19.0 & 2.4 \\
\hline $\begin{array}{l}11525-1408 \\
(11)\end{array}$ & HDS 1 & 676 & $\begin{array}{r}16.36 \\
\pm 2.60\end{array}$ & $\begin{array}{r}2000.45 \\
\pm 2.32\end{array}$ & $\begin{array}{r}0.643 \\
\pm 0.167\end{array}$ & $\begin{array}{r}313.1 \\
\pm 30.4\end{array}$ & $\begin{array}{r}0.134 \\
\pm 0.017\end{array}$ & $\begin{array}{r}42.8 \\
\pm 8.9\end{array}$ & $\begin{array}{r}327.3 \\
\pm 44.6\end{array}$ & 4 & 15.6 & 2.4 \\
\hline $\begin{array}{l}12485-1543 \\
(12)\end{array}$ & WSI & $74 \mathrm{Aa}, \mathrm{Ab}$ & $\begin{array}{r}2.687 \\
\pm 0.008\end{array}$ & $\begin{array}{r}2011.179 \\
\pm 0.020\end{array}$ & $\begin{array}{r}0.500 \\
\pm 0.020\end{array}$ & $\begin{array}{r}152.1 \\
\pm 3.1\end{array}$ & $\begin{array}{r}0.080 \\
\pm 0.004\end{array}$ & $\begin{array}{r}51.0 \\
\pm 4.1\end{array}$ & $\begin{array}{r}147.0 \\
\pm 2.4\end{array}$ & 3 & 42.0 & 1.0 \\
\hline $\begin{array}{l}17066+0039 \\
(13)\end{array}$ & TOK & $52 \mathrm{Ba}, \mathrm{Bb}$ & $\begin{array}{r}7.00 \\
\pm 0.70\end{array}$ & $\begin{array}{r}2012.94 \\
\pm 0.10\end{array}$ & $\begin{array}{r}0.430 \\
\pm 0.032\end{array}$ & $\begin{array}{r}56.7 \\
\pm 111\end{array}$ & $\begin{array}{r}0.069 \\
\pm 0.004\end{array}$ & $\begin{array}{l}13.7 \\
\pm 16\end{array}$ & $\begin{array}{l}130.0 \\
\pm 119\end{array}$ & 4 & 17.3 & 1.3 \\
\hline $\begin{array}{l}21368-3043 \\
(14)\end{array}$ & VOU & $35 \mathrm{AB}$ & $\begin{array}{r}19.819 \\
\pm 0.010\end{array}$ & $\begin{array}{r}2003.751 \\
\pm 0.093\end{array}$ & $\begin{array}{r}0.50 \\
\pm 0.00\end{array}$ & $\begin{array}{r}114.0 \\
\pm 1.4\end{array}$ & $\begin{array}{r}0.145 \\
\pm 0.002\end{array}$ & $\begin{array}{r}114.3 \\
\pm 1.0\end{array}$ & $\begin{array}{r}130.0 \\
\pm 0.0\end{array}$ & 3 & 16.1 & 1.0 \\
\hline $\begin{array}{l}23100-4252 \\
(15)\end{array}$ & DON1 & 1042 & $\begin{array}{r}100.9 \\
\pm 4.0\end{array}$ & $\begin{array}{r}1995.1 \\
\pm 4.9\end{array}$ & $\begin{array}{r}0.626 \\
\pm 0.125\end{array}$ & $\begin{array}{r}249 \\
\pm 22\end{array}$ & $\begin{array}{r}0.779 \\
\pm 0.133\end{array}$ & $\begin{array}{r}44.4 \\
\pm 13.8\end{array}$ & $\begin{array}{r}105.6 \\
\pm 15.2\end{array}$ & 5 & 29.9 & 1.7 \\
\hline
\end{tabular}


TABLE 7

Revised Orbital Elements

\begin{tabular}{|c|c|c|c|c|c|c|c|c|c|c|}
\hline WDS & $\begin{array}{c}\text { Discoverer } \\
\text { Designation }\end{array}$ & $\begin{array}{c}P \\
(\mathrm{yr})\end{array}$ & $\begin{array}{l}T_{\circ} \\
(\mathrm{yr})\end{array}$ & $e$ & $\begin{array}{l}\Omega \\
(0)\end{array}$ & $\begin{array}{c}a \\
\left({ }^{\prime \prime}\right)\end{array}$ & $\begin{array}{l}i \\
(0)\end{array}$ & $\begin{array}{l}\omega \\
(0)\end{array}$ & Gr. & $\begin{array}{c}\text { Published Orbit } \\
\text { Reference }\end{array}$ \\
\hline $01071-0036$ & HDS $144 \mathrm{AB}$ & 72.6 & 2073.1 & 0.237 & 281.9 & 0.365 & 138.2 & 30. & 4 & Cvetkovic (2011) \\
\hline $04107-0452$ & A 2801 & $\begin{array}{r}20.42 \\
\pm 0.12\end{array}$ & $\begin{array}{r}1952.61 \\
\pm 0.36\end{array}$ & $\begin{array}{r}0.887 \\
\pm 0.031\end{array}$ & $\begin{array}{r}156.6 \\
\pm 3.5\end{array}$ & $\begin{array}{r}0.194 \\
\pm 0.018\end{array}$ & $\begin{array}{r}71.5 \\
\pm 2.2\end{array}$ & $\begin{array}{r}70.4 \\
\pm 3.8\end{array}$ & 2 & Baize (1986) \\
\hline $04258+1800$ & COU 2682 & 54.8 & 1955.6 & 0.163 & 328.5 & 0.348 & 68.7 & 11.6 & 4 & Docobo \& Tamazian (2007) \\
\hline $05059-1355$ & A 3009 & 284 & 1957.2 & 0.237 & 244.9 & 1.283 & 40.1 & 294.1 & 5 & Erceg (1985) \\
\hline $05135+0158$ & $\mathrm{STT} \quad 517 \mathrm{AB}$ & 987. & 1925.3 & 0.935 & 138. & 0.77 & 18. & 304. & 4 & Mason et al. (2009) \\
\hline $05164-0139$ & A $\quad 844$ & $\begin{array}{r}168.8 \\
\pm 12.6\end{array}$ & $\begin{array}{r}1997.4 \\
\pm 6.6\end{array}$ & $\begin{array}{r}0.160 \\
\pm 0.033\end{array}$ & $\begin{array}{r}191.7 \\
\pm 3.1\end{array}$ & $\begin{array}{r}0.234 \\
\pm 0.006\end{array}$ & $\begin{array}{r}50.1 \\
\pm 3.4\end{array}$ & $\begin{array}{r}337.1 \\
\pm 15.5\end{array}$ & 3 & Hartkopf \& Mason (2001a) \\
\hline $05165-2106$ & DON 97 & 258.0 & 1952.8 & 0.264 & 165.4 & 0.457 & 133.8 & 205.7 & 4 & Seymour et al. (2002) \\
\hline $05320-0018$ & HEI $42 \mathrm{Aa}, \mathrm{Ab}$ & 346. & 1964.8 & 0.812 & 148.4 & 0.423 & 107. & 260. & 4 & Mason et al. (2009) \\
\hline $05525-0217$ & HDS 787 & $\begin{array}{r}11.768 \\
\pm 0.058\end{array}$ & $\begin{array}{r}2000.01 \\
\pm 0.07\end{array}$ & $\begin{array}{r}0.238 \\
\pm 0.010\end{array}$ & $\begin{array}{r}153.5 \\
\pm 1.7\end{array}$ & $\begin{array}{r}0.120 \\
\pm 0.002\end{array}$ & $\begin{array}{l}53.4 \\
\pm 1.9\end{array}$ & $\begin{array}{r}274.5 \\
\pm 3.1\end{array}$ & 3 & Mason et al. (2010) \\
\hline $06003-3102$ & HU $1399 \mathrm{AB}$ & $\begin{array}{r}67.45 \\
\pm 0.14\end{array}$ & $\begin{array}{r}1997.85 \\
\pm 0.17\end{array}$ & $\begin{array}{r}0.516 \\
\pm 0.013\end{array}$ & $\begin{array}{r}126.7 \\
\pm 0.5\end{array}$ & $\begin{array}{r}0.946 \\
\pm 0.010\end{array}$ & $\begin{array}{r}101.2 \\
\pm 0.4\end{array}$ & $\begin{array}{r}278.5 \\
\pm 0.5\end{array}$ & 2 & Tokovinin et al. (2005) \\
\hline $06003-3102$ & TOK $\quad 9 \mathrm{CE}$ & $\begin{array}{r}22.95 \\
\pm 3.21\end{array}$ & $\begin{array}{r}2016.50 \\
\pm 0.70\end{array}$ & $\begin{array}{r}0.186 \\
\pm 0.094\end{array}$ & $\begin{array}{r}146.6 \\
\pm 0.9\end{array}$ & $\begin{array}{r}0.441 \\
\pm 0.031\end{array}$ & $\begin{array}{r}97.8 \\
\pm 1.0\end{array}$ & $\begin{array}{r}193 \\
\pm 19\end{array}$ & 3 & Hartkopf et al. (2012) \\
\hline $06048-4828$ & DUN 23 & 915 & 2005 & 0.427 & 121.4 & 4.578 & 63.6 & 2.0 & 5 & Scardia (2001) \\
\hline $06122-3645$ & RST 4800 & 174. & 2002.9 & 0.505 & 191.0 & 0.275 & 131.3 & 248.4 & 4 & Seymour et al. (2002) \\
\hline $06253+0130$ & FIN 343 & $\begin{array}{r}69.7 \\
\pm 5.1\end{array}$ & $\begin{array}{r}2020.0 \\
\pm 1.8\end{array}$ & $\begin{array}{r}0.441 \\
\pm 0.050\end{array}$ & $\begin{array}{l}234 \\
\pm 28\end{array}$ & $\begin{array}{r}0.131 \\
\pm 0.004\end{array}$ & $\begin{array}{r}156.5 \\
\pm 8.4\end{array}$ & $\begin{aligned} & 53 \\
\pm & 23\end{aligned}$ & 3 & Docobo \& Andrade (2013) \\
\hline $06545-2734$ & 706 & 262. & 1945.8 & 0.734 & 90.0 & 0.675 & 66.9 & 68.4 & 4 & Dommanget (1979) \\
\hline $07015-0942$ & $3042 \mathrm{AB}$ & $\begin{array}{r}99.3 \\
\pm 2.3\end{array}$ & $\begin{array}{r}2007.57 \\
\pm 0.27\end{array}$ & $\begin{array}{r}0.621 \\
\pm 0.013\end{array}$ & $\begin{array}{r}223.7 \\
\pm 2.4\end{array}$ & $\begin{array}{r}0.290 \\
\pm 0.007\end{array}$ & $\begin{array}{r}54.0 \\
\pm 2.5\end{array}$ & $\begin{array}{r}113.1 \\
\pm 3.3\end{array}$ & 3 & Olevic \& Jovanovic (2001) \\
\hline $07043-0303$ & 519 & $\begin{array}{l}46.68 \\
\pm 0.48\end{array}$ & $\begin{array}{r}2015.47 \\
\pm 1.06\end{array}$ & $\begin{array}{r}0.661 \\
\pm 0.039\end{array}$ & $\begin{array}{r}99.8 \\
\pm 1.2\end{array}$ & $\begin{array}{r}0.272 \\
\pm 0.014\end{array}$ & $\begin{array}{l}99.5 \\
\pm 2.3\end{array}$ & $\begin{array}{r}34.3 \\
\pm 5.8\end{array}$ & 2 & Docobo \& Ling (2009) \\
\hline $07294-1500$ & $\mathrm{STF} 1104 \mathrm{AB}$ & 729 & 2000 & 0.142 & 157.0 & 2.543 & 38.1 & 236 & 5 & Mason et al. (2004) \\
\hline $07324-3558$ & RST 4855 & $\begin{array}{r}116.7 \\
\pm 5.5\end{array}$ & $\begin{array}{r}1991.28 \\
\pm 0.31\end{array}$ & $\begin{array}{r}0.832 \\
\pm 0.018\end{array}$ & $\begin{array}{r}168.8 \\
\pm 9.3\end{array}$ & $\begin{array}{r}0.212 \\
\pm 0.006\end{array}$ & $\begin{array}{r}34.6 \\
\pm 4.2\end{array}$ & $\begin{array}{r}44.6 \\
\pm 9.4\end{array}$ & 3 & Mante (2001) \\
\hline $07411-0124$ & A 1968 & 146.2 & 1995.8 & 0.611 & 143.4 & 0.416 & 115.6 & 274.0 & 4 & Scardia (1983) \\
\hline $07430-1704$ & HU $\quad 710$ & $\begin{array}{r}158.7 \\
\pm 4.8\end{array}$ & $\begin{array}{r}1952.15 \\
\pm 0.77\end{array}$ & $\begin{array}{r}0.622 \\
\pm 0.015\end{array}$ & $\begin{array}{r}111.6 \\
\pm 4.2\end{array}$ & $\begin{array}{r}0.363 \\
\pm 0.007\end{array}$ & $\begin{array}{r}137.7 \\
\pm 1.8\end{array}$ & $\begin{array}{r}249.8 \\
\pm 4.0\end{array}$ & 3 & Heintz (1981) \\
\hline $08173-0522$ & 337 & 207. & 1900.7 & 0.312 & 215.1 & 0.465 & 129.6 & 311.7 & 4 & Heintz (1978a) \\
\hline $08213-0136$ & STF 1216 & $\begin{array}{l}402 \\
\pm 15\end{array}$ & $\begin{array}{l}2322 \\
\pm 26\end{array}$ & $\begin{array}{r}0.109 \\
\pm 0.018\end{array}$ & $\begin{array}{r}264.1 \\
\pm 4.8\end{array}$ & $\begin{array}{r}0.565 \\
\pm 0.016\end{array}$ & $\begin{array}{r}31.7 \\
\pm 1.6\end{array}$ & $\begin{array}{r}310.8 \\
\pm 9.5\end{array}$ & 3 & Docobo et al. (1994) \\
\hline $08275-5501$ & FIN 116 & $\begin{array}{r}125.8 \\
\pm 2.4\end{array}$ & $\begin{array}{r}1994.22 \\
\pm 0.25\end{array}$ & $\begin{array}{r}0.524 \\
\pm 0.008\end{array}$ & $\begin{array}{r}241.0 \\
\pm 1.3\end{array}$ & $\begin{array}{r}0.273 \\
\pm 0.003\end{array}$ & $\begin{array}{r}121.5 \\
\pm 1.3\end{array}$ & $\begin{array}{r}260.2 \\
\pm 1.8\end{array}$ & 3 & Cvetkovic \& Ninkovic (2010) \\
\hline $09125-4032$ & 1115 & 646 & 1998.32 & 0.776 & 279.6 & 1.024 & 114.2 & 41.1 & 5 & Seymour et al. (2002) \\
\hline $09243-3926$ & FIN 348 & $\begin{array}{r}39.73 \\
\pm 0.75\end{array}$ & $\begin{array}{r}1983.43 \\
\pm 0.56\end{array}$ & $\begin{array}{r}0.529 \\
\pm 0.018\end{array}$ & $\begin{array}{r}61.5 \\
\pm 4.2\end{array}$ & $\begin{array}{r}0.126 \\
\pm 0.002\end{array}$ & $\begin{array}{r}147.4 \\
\pm 2.4\end{array}$ & $\begin{array}{r}300.8 \\
\pm 2.9\end{array}$ & 3 & Mason et al. (2010) \\
\hline $09264-4215$ & 1122 & 316. & 2206 & 0.23 & 153. & 0.23 & 54 & 291. & 4 & Seymour et al. (2002) \\
\hline $10361-2641$ & BU 411 & $\begin{array}{r}158.5 \\
\pm 2.4\end{array}$ & $\begin{array}{r}1948.82 \\
\pm 0.53\end{array}$ & $\begin{array}{r}0.759 \\
\pm 0.010\end{array}$ & $\begin{array}{r}149.5 \\
\pm 1.5\end{array}$ & $\begin{array}{r}0.880 \\
\pm 0.013\end{array}$ & $\begin{array}{r}127.7 \\
\pm 1.7\end{array}$ & $\begin{array}{r}43.2 \\
\pm 1.9\end{array}$ & 3 & Scardia (2001) \\
\hline $10370-0850$ & $556 \mathrm{AB}$ & 285. & 2026 & 0.144 & 259. & 1.428 & 48.1 & 314 & 5 & Popovic (1978) \\
\hline $10446+0530$ & 2771 & 549. & 1993.47 & 0.834 & 288.6 & 1.016 & 131.4 & 52.8 & 5 & Heintz (1997) \\
\hline $10468-4925$ & 155 & 149.3 & 1945.4 & 0.969 & 283.0 & 2.319 & 66.0 & 291.7 & 4 & Heintz (1986a) \\
\hline $10592-8133$ & 212 & 246. & 2002.0 & 0.510 & 191.1 & 0.599 & 49.2 & 118.8 & 4 & Seymour et al. (2002) \\
\hline $11221-2447$ & $507 \mathrm{AB}$ & 214.0 & 2023.1 & 0.400 & 184.2 & 1.030 & 88.1 & 249.1 & 4 & Tokovinin (1999) \\
\hline $11395-6524$ & $1705 \mathrm{AB}$ & $\begin{array}{r}96.8 \\
\pm 2.4\end{array}$ & $\begin{array}{r}2003.55 \\
\pm 0.29\end{array}$ & $\begin{array}{r}0.634 \\
\pm 0.015\end{array}$ & $\begin{array}{r}259.8 \\
\pm 1.5\end{array}$ & $\begin{array}{r}0.276 \\
\pm 0.004\end{array}$ & $\begin{array}{r}60.9 \\
\pm 2.0\end{array}$ & $\begin{array}{r}92.4 \\
\pm 1.5\end{array}$ & 3 & Zirm (2008) \\
\hline $11495-4604$ & FIN 366 & $\begin{array}{r}67.6 \\
\pm 13.3\end{array}$ & $\begin{array}{r}1966.3 \\
\pm 2.5\end{array}$ & $\begin{array}{r}0.595 \\
\pm 0.097\end{array}$ & $\begin{array}{r}155.1 \\
\pm 4.8\end{array}$ & $\begin{array}{r}0.237 \\
\pm 0.056\end{array}$ & $\begin{array}{r}75.4 \\
\pm 4.7\end{array}$ & $\begin{aligned} 71 \\
\pm 16\end{aligned}$ & 3 & Cvetkovic (2008) \\
\hline $12018-3439$ & I $\quad 215$ & 327.2 & 2005.2 & 0.501 & 92.7 & 1.345 & 106.6 & 47.5 & 4 & Heintz (1997) \\
\hline $12036-3901$ & SEE 143 & $\begin{array}{r}111.0 \\
\pm 1.4\end{array}$ & $\begin{array}{r}1913.0 \\
\pm 1.3\end{array}$ & $\begin{array}{r}0.579 \\
\pm 0.012\end{array}$ & $\begin{array}{r}211.0 \\
\pm 8.5\end{array}$ & $\begin{array}{r}0.673 \\
\pm 0.009\end{array}$ & $\begin{array}{r}155.3 \\
\pm 2.1\end{array}$ & $\begin{array}{r}285.4 \\
\pm 7.1\end{array}$ & 3 & Söderhielm (1999) \\
\hline $12283-6146$ & RST $4499 \mathrm{BC}$ & $\begin{array}{r}28.01 \\
\pm 0.16\end{array}$ & $\begin{array}{r}1990.07 \\
\pm 0.33\end{array}$ & $\begin{array}{r}0.170 \\
\pm 0.009\end{array}$ & $\begin{array}{r}161.5 \\
\pm 8.8\end{array}$ & $\begin{array}{r}0.229 \\
\pm 0.003\end{array}$ & $\begin{array}{r}154.4 \\
\pm 3.0\end{array}$ & $\begin{array}{r}73.3 \\
\pm 6.7\end{array}$ & 3 & Heintz (1997) \\
\hline $13149-1122$ & $\mathrm{RST} 3829 \mathrm{Aa}, \mathrm{Ab}$ & 122.7 & 1985.11 & 0.449 & 120.8 & 0.762 & 64.2 & 285.3 & 4 & Heintz (1997) \\
\hline
\end{tabular}


TABLE 7-Continued

\begin{tabular}{|c|c|c|c|c|c|c|c|c|c|c|}
\hline WDS & $\begin{array}{c}\text { Discoverer } \\
\text { Designation }\end{array}$ & $\begin{array}{c}P \\
(\mathrm{yr})\end{array}$ & $\begin{array}{l}T_{\circ} \\
(\mathrm{yr})\end{array}$ & $e$ & $\begin{array}{l}\Omega \\
(0)\end{array}$ & $\begin{array}{c}a \\
\left({ }^{\prime \prime}\right)\end{array}$ & $\begin{array}{l}i \\
\text { (o) }\end{array}$ & $\begin{array}{c}\omega \\
(0)\end{array}$ & Gr. & $\begin{array}{l}\text { Published Orbit } \\
\text { Reference }\end{array}$ \\
\hline $13520-3137$ & BU 343 & 280. & 1996.61 & 0.656 & 177.5 & 1.268 & 130.2 & 231.9 & 4 & Seymour et al. (2002) \\
\hline $14373-4608$ & FIN $318 \mathrm{Aa}, \mathrm{Ab}$ & 141. & 1973.0 & 0.750 & 189.9 & 0.216 & 122.0 & 272.8 & 4 & Docobo \& Andrade (2013) \\
\hline $17157-0949$ & A $2592 \mathrm{AB}$ & $\begin{array}{r}128.0 \\
\pm 4.9\end{array}$ & $\begin{array}{r}2016.11 \\
\pm 0.57\end{array}$ & $\begin{array}{r}0.395 \\
\pm 0.012\end{array}$ & $\begin{array}{r}199.2 \\
\pm 4.3\end{array}$ & $\begin{array}{r}0.334 \\
\pm 0.012\end{array}$ & $\begin{array}{r}143.4 \\
\pm 3.0\end{array}$ & $\begin{array}{r}100.6 \\
\pm 4.1\end{array}$ & 3 & Heintz (1996) \\
\hline $21198-2621$ & $\mathrm{BU} \quad 271 \mathrm{AB}$ & $\begin{array}{r}157.5 \\
\pm 7.5\end{array}$ & $\begin{array}{r}1862.9 \\
\pm 4.8\end{array}$ & $\begin{array}{r}0.898 \\
\pm 0.062\end{array}$ & $\begin{array}{r}217.6 \\
\pm 12.2\end{array}$ & $\begin{array}{r}2.46 \\
\pm 0.38\end{array}$ & $\begin{array}{r}60.6 \\
\pm 3.2\end{array}$ & $\begin{array}{r}232.8 \\
\pm 14.2\end{array}$ & 3 & Jasinta (1997) \\
\hline $22288-0001$ & $\mathrm{EBE} \quad 1 \mathrm{Aa}, \mathrm{Ab}$ & 25.70 & 2003.6 & 0.590 & 32.4 & 0.434 & 66.2 & 348 & 5 & Scardia et al. (2010) \\
\hline $23474-7118$ & FIN $375 \mathrm{Aa}, \mathrm{Ab}$ & 82. & 2037. & 0.515 & 139. & 0.180 & 60.3 & 246.9 & 4 & Olevic \& Cvetkovic (2004b) \\
\hline
\end{tabular}

